ICRR-Report-508-2004-6

OU-TAP-234

TU-727

astro-ph/0408426

August, 2004

\title{
Big-Bang Nucleosynthesis and Hadronic Decay of Long-Lived Massive Particles
}

\author{
Masahiro Kawasaki ${ }^{(a)}$, Kazunori Kohri ${ }^{(b)}$ and Takeo Moroi ${ }^{(c)}$ \\ (a) Institute for Cosmic Ray Research, University of Tokyo \\ Kashiwa, Chiba 277-8582, Japan \\ ${ }^{(b)}$ Department of Earth and Space Science, Graduate School of Science \\ Osaka University, Toyonaka, Osaka 560-0043, Japan \\ (c) Department of Physics, Tohoku University, Sendai 980-8578, Japan
}

\begin{abstract}
We study the big-bang nucleosynthesis (BBN) with the long-lived exotic particle, called $X$. If the lifetime of $X$ is longer than $\sim 0.1$ sec, its decay may cause nonthermal nuclear reactions during or after the $\mathrm{BBN}$, altering the predictions of the standard BBN scenario. We pay particular attention to its hadronic decay modes and calculate the primordial abundances of the light elements. Using the result, we derive constraints on the primordial abundance of $X$.

Compared to the previous studies, we have improved the following points in our analysis: The JETSET 7.4 Monte Carlo event generator is used to calculate the spectrum of hadrons produced by the decay of $X$; The evolution of the hadronic shower is studied taking account of the details of the energy-loss processes of the nuclei in the thermal bath; We have used the most recent observational constraints on the primordial abundances of the light elements; In order to estimate the uncertainties, we have performed the Monte Carlo simulation which includes the experimental errors of the cross sections and transfered energies.

We will see that the non-thermal productions of $\mathrm{D},{ }^{3} \mathrm{He},{ }^{4} \mathrm{He}$ and ${ }^{6} \mathrm{Li}$ provide stringent upper bounds on the primordial abundance of late-decaying particle, in particular when the hadronic branching ratio of $X$ is sizable. We apply our results to the gravitino problem, and obtain upper bound on the reheating temperature after inflation.
\end{abstract}




\section{Introduction}

In modern cosmology, big-bang nucleosynthesis (BBN) is one of the most important subjects. In the standard scenario, neutrons freeze out from the thermal bath when the cosmic temperature is $\sim 0.7 \mathrm{MeV}$ and then the light elements (i.e., D, ${ }^{4} \mathrm{He},{ }^{7} \mathrm{Li}$, and so on) are synthesized subsequently. As we will discuss in the next section, prediction of the standard $\mathrm{BBN}(\mathrm{SBBN})$ scenario is in a reasonable agreement with the observations.

Predicted abundances of the light elements are, however, very sensitive to the cosmological scenarios. In particular, if we consider exotic cosmological scenarios based on physics beyond the standard model, theoretical predictions on the light-element abundances may be too much affected to be consistent with the observations. Thus, the BBN provides significant constraints on the new particles which change the cosmological evolution at the cosmic time $t \sim 10^{-2}-10^{12}$ sec. If we consider physics beyond the standard model, there exist various candidates of such exotic particles. (Hereafter, we call such particle as $X$.)

One example of the long-lived particles is the gravitino in supergravity theory [1. Gravitino acquires mass from the effect of the supersymmetry breaking. In addition, its interactions are suppressed by inverse powers of the gravitational scale and hence its lifetime becomes very long (if it is unstable). In particular, for supersymmetric models with the gravitino mass $m_{3 / 2} \sim \mathcal{O}\left(10^{2-3}\right) \mathrm{GeV}$, lifetime of the gravitino becomes much longer than $1 \mathrm{sec}$ and its decay may significantly affect the light-element abundances. (This is called the "gravitino problem.") Thus, the BBN provides substantial constraints on the properties of the gravitino and also on the cosmological scenarios. (For more details of the gravitino problem, see [2] and references therein.)

In addition, even for the case where the gravitino is the lightest superparticle (LSP), the next-to-the lightest superparticle (NLSP) has long lifetime since the NLSP decays into its superpartner and the gravitino. The BBN imposes significant constraints on the case where the NLSP is the neutralino or the scalar- $\tau$ [3, 4]. Furthermore, moduli fields in the superstring theory are another candidates of $X$. Some of the moduli fields may acquire non-vanishing amplitude in the early universe. If so, their coherent oscillation may decay at a very late stage of the evolution of the universe.

The exotic particles listed above are some of the famous examples and, if one considers particle-physics models beyond the standard model, there may exist long-lived particles which may affect the BBN. Thus, it is important to study the BBN scenario with latedecaying particles. Such studies have been done by various groups [5, 6, 17, 8, 9, 10, 11, 12, 13, 14, 15, 16, 76, 17.

In most of the previous studies (except 6, 7, 8, 15]), however, hadronic decay modes of $X$ were ignored although, for many of the candidates of the long-lived exotic particles, it is expected that the hadronic branching ratio is sizable. For example, even if $X$ dominantly decays into photon (and something else), the hadronic branching ratio is expected to be non-vanishing since the (virtual) photon can be converted to the quark anti-quark

pair. In this case, the hadronic branching ratio is estimated to be at least $\sim \alpha_{\mathrm{em}} / 4 \pi \sim$ $10^{-(2-3)}$ (with $\alpha_{\mathrm{em}}$ being the fine structure constant), unless the hadronic decay mode 
is kinematically suppressed. Of course, if $X$ directly decays into quarks and/or gluons, hadronic branching ratio can be close to 1 .

If the massive particles decay into quarks or gluons during/after the BBN epoch, many mesons (mostly pions) and nucleons are produced. The emitted hadrons lose their energy via the electromagnetic interactions and scatter off the background nuclei. The emitted hadrons affect the BBN via two effects. One is the "inter-conversion" effect; if $X$ decays at relatively early stage of the BBN (i.e., $t \lesssim 10^{2} \mathrm{sec}$ ), emitted hadrons may change the neutron-to-proton ratio. On the contrary, at the later stage of the BBN (i.e., $t \gtrsim 10^{2} \mathrm{sec}$ ), hadrodissociation processes are caused by energetic hadrons generated by the decay of $X$. Due to these effects, light-element abundances can be significantly affected.

The effects of the inter-conversion was first studied in [7], whose results were also applied to some of the topics in the early universe in [18, 19]. In these works, however, it is simply assumed that all of emitted hadrons are effectively stopped even when the cosmic time is longer than $t \sim 10^{2}$ sec. Because the energy loss process becomes inefficient at $t \gtrsim 10^{2} \mathrm{sec}$, their assumption is inappropriate, and we should correctly simulate the stopping of the hadrons through the electromagnetic interactions with background plasma. On the other hand, effects of the hadrodissociation processes were studied in 8 . This study is, however, based on theoretical and experimental information which can be improved with our current knowledge.

Importantly, after these studies, there have been various theoretical, experimental and observational progresses to study the BBN scenario with long-lived exotic particles. First, with the progresses in the high-energy experiments, now we have better information on the hadron fragmentation processes. In particular, we use the JETSET 7.4 Monte Carlo event generator 20] to estimate the distributions of the nucleons and mesons produced by the hadronic decay of $X$. In addition, we have more experimental data of the hadronnucleon cross sections and energy distributions of the hadronic particles generated by the hadrodissociation processes. With these improvements, we can perform a better study of the evolution of the hadronic shower. Furthermore, observational constraints on the primordial abundances of the light elements are also improved.

The purpose of this paper is to investigate the BBN scenario with the long-lived exotic particle using the currently available best knowledges on particle physics, nuclear physics, and astrophysics, paying particular attention to hadronic decay modes of $X$. We calculate the abundances of the light elements including relevant hadronic scattering processes (as well as photodissociation processes). In our study of the evolution of the hadronic showers, the basic framework is the same as that used in 88 although there are several modifications. The most important improvements are as follows. (i) We carefully take into account the energy loss processes for high-energy nuclei through the scattering with background photons or electrons. In particular, dependence on the cosmic temperature, the initial energies of nuclei, and the background ${ }^{4} \mathrm{He}$ abundance are considered. (ii) We use available data of cross sections and transfered energies of elastic and inelastic hadronhadron scattering processes as much as possible. (iii) The time evolution of the energy distribution functions of high-energy nuclei are computed with proper energy resolution. 
(iv) The JETSET 7.4 Monte Carlo event generator [20] is used to obtain the initial spectrum of hadrons produced by the decay of $X$. (v) The most resent data of observational light element abundances are adopted. (vi) We estimate uncertainties with Monte Carlo simulation which includes the experimental errors of the cross sections and transfered energies, and uncertainty of the baryon to photon ratio [21].

This paper is the full-length version of our recent letter [17]. This paper is organized as follows. In the next section, we briefly review the current status of the observations and SBBN. Then, in Section 3, we give an overview of the decay of massive particles and its cosmological effects. In Section 4, we give a brief overview of the photodissociation process. We outline the hadronic decay scenarios in Section [5. In Section 6] we introduce the formulations and computations of inter-conversion effects between background $p$ and $n$ by hadrons at earlier epochs in the hadron injection scenario. In Section 7 we discuss the destruction and production processes of light elements in hadrodissociation scenario. We also consider the non-thermal production processes of Lithium and Beryllium in hadronic decay scenario in Section 8. In Section 9] we compare the theoretical predictions with the observations in hadronic and radiative decay scenario for general massive particles. Our main results are shown in this section; if the reader is mostly interested in the resultant constraints, see this section (in particular, see Figs. 38 - 42). Then, in Section [10 we apply our results to the case of decaying gravitinos in supergravity. Section 11 is devoted to the conclusions and discussion.

\section{Current Status: Observation and SBBN}

\subsection{Current status of observations}

First we briefly summarize the current status of the observational light element abundances. The errors of the following observational values are at $1 \sigma$ level unless otherwise stated.

The primordial value of the ratio $n_{\mathrm{D}} / n_{\mathrm{H}}$ is measured in the high redshift QSO absorption systems. (Here and hereafter, $n_{A_{i}}$ denotes the number density of the nucleus $A_{i}$.) Recently a new deuterium data was obtained from observation of the absorption system at the redshift $z=2.525659$ towards Q1243+3047 [22] including improved modeling of the continuum level, the Ly- $\alpha$ forest and the velocity structure of the absorption systems. The reported value of the deuterium abundance by using Keck-I HIRES, was relatively low, $\left(n_{\mathrm{D}} / n_{\mathrm{H}}\right)^{\text {obs }}=\left(2.42_{-0.25}^{+0.35}\right) \times 10^{-5}$. Combined with the previous data [23, 24, 25, 26], it is reported that the primordial abundance is given by $\# 1$

$$
\left(n_{\mathrm{D}} / n_{\mathrm{H}}\right)^{\mathrm{obs}}=\left(2.78_{-0.38}^{+0.44}\right) \times 10^{-5} .
$$

\footnotetext{
${ }^{\# 1}$ Note that higher deuterium abundance in relatively low redshift absorption systems at $\mathrm{z}=0.701$ was also reported: $n_{\mathrm{D}} / n_{\mathrm{H}}=(2.0 \pm 0.5) \times 10^{-4}$ [27. Based on another independent observation of the clouds, however, it is claimed that the observed absorption is not due to $\mathrm{D}$ although there are still some uncertainties. Thus, we do not adopt the "High D" primordial abundance in this paper.
} 
(Here and hereafter, the superscript "obs" is used for the primordial values inferred by the observations.)

The primordial abundance of ${ }^{4} \mathrm{He}$ is inferred from the recombination lines from the low metallicity extragalactic HII regions. One obtains the primordial value of the ${ }^{4} \mathrm{He}$ mass fraction $Y$ by regressing to the zero metallicity $\mathrm{O} / \mathrm{H} \rightarrow 0$ for the observational data. Based on the reanalysis of Fields and Olive [28], which takes account of the effect of the HeI absorption, the primordial mass fraction is given by

$$
Y^{\mathrm{obs}}(\mathrm{FO})=0.238 \pm(0.002)_{\mathrm{stat}} \pm(0.005)_{\mathrm{syst}},
$$

where the first and second errors are the statistical and systematic ones, respectively. On the contrary, Izotov and Thuan [29] reported a slightly higher value :

$$
Y^{\mathrm{obs}}(\mathrm{IT})=0.242 \pm(0.002)_{\text {stat }}\left( \pm(0.005)_{\text {syst }}\right),
$$

where we have added the systematic errors following Refs. 30, 31, 32]. Since there exists sizable difference between the results of two groups, we use two values $Y^{\text {obs }}(\mathrm{FO})$ and $Y^{\mathrm{obs}}(\mathrm{IT})$. \#2

As for ${ }^{7} \mathrm{Li}$, it is widely believed that the primordial abundance of ${ }^{7} \mathrm{Li}$ can be determined using Pop II old halo stars with temperature higher than $\sim 6000 \mathrm{~K}$ and with low metallicity. We adopt the recent measurements by Ref. 34]:

$$
\log _{10}\left[\left(n_{7 \mathrm{Li}} / n_{\mathrm{H}}\right)^{\text {obs }}\right]=-9.66 \pm(0.056)_{\text {stat }}\left( \pm(0.3)_{\text {add }}\right) .
$$

Here we adopt the additional uncertainty for fear that the ${ }^{7} \mathrm{Li}$ in the halo stars might have been supplemented (by production in cosmic-ray interactions) or depleted (in stars) 35. Result given in Eq. (2.4) corresponds to $n_{7} \mathrm{Li} / n_{\mathrm{H}}=\left(2.19_{-0.27}^{+0.30}\right) \times 10^{-10}$ and $\left(2.19_{-1.1}^{+2.2}\right) \times 10^{-10}$ for the cases without and with the additional systematic errors.

Note that it was claimed that there can be a significant dependence of $n_{\mathrm{Li}}$ on $\mathrm{Fe}$ abundance in the low metallicity region [36]. In addition, assuming that this trend is due to the cosmic ray interactions, Ref. [37] inferred that the primordial value is $n_{7 \mathrm{Li}} / n_{\mathrm{H}}=$ $\left(1.23_{-0.32}^{+0.68}\right) \times 10^{-10}$. Since the precise determination of the primordial abundance from the observations is out of the scope of this paper, we conservatively adopt the value in Eq. (2.4) with large uncertainties in this paper. This is also justified from the point of the view of deriving conservative constraints; with the primordial abundance of ${ }^{7} \mathrm{Li}$ given by [37, discrepancy between the values of the baryon-to-photon ratio $\eta$ determined by the SBBN and that by the observations of the cosmic microwave background (CMB) anisotropies becomes worse. (See the next subsection.)

For ${ }^{6} \mathrm{Li}$, it is much more difficult to determine its primordial abundance since ${ }^{6} \mathrm{Li}$ is much rarer than ${ }^{7} \mathrm{Li}$. Unfortunately, data is insufficient and ${ }^{6} \mathrm{Li}$ abundance cannot be

\footnotetext{
\#2Recently, Olive and Skillman [33 reanalyzed Izotov and Thuan's data and found large systematic errors. However, their method may not adequate since they obtained unphysical HI and HeI absorptions for some of HII regions and cannot determine the electron density well by their method. It is premature to judge its reliability. Thus, we do not adopt their result here.
} 
reliably determined. However, because it is generally believed that the evolution of ${ }^{6} \mathrm{Li}$ is dominated by the production through the cosmic ray spallation (i.e., reactions of cosmic rays with the interstellar medium), we can set an upper bound on the ratio $n_{6} \mathrm{Li} / n_{7} \mathrm{Li}$. The models of the nucleosynthesis through the cosmic ray spallation were intrinsically required to simultaneously explain the whole observational Li-Be-B abundances [38, 39, 40]. On the other hand, recently it was claimed that the observational ${ }^{6} \mathrm{Li}$ abundance in halo stars is too abundant from the point of view of the cosmic ray energy if ${ }^{9} \mathrm{Be}$ is fitted by the model of the cosmic-ray metal [41]. Therefore, there seems to be some uncertainties in the models of the cosmic ray spallation. ${ }^{\# 3}$ In this situation, at least it would be safe to assume that ${ }^{6} \mathrm{Li}$ abundance increases as the metallicity increases. Today we observe only the ${ }^{6} \mathrm{Li}$ to ${ }^{7} \mathrm{Li}$ ratio in low-metallicity $([\mathrm{Fe} / \mathrm{H}] \leq-2.0)$ halo stars 43 ,

$$
\left(n_{6 \mathrm{Li}} / n_{7 \mathrm{Li}}\right)^{\text {halo }}=0.05 \pm 0.02(2 \sigma) .
$$

We take this value as an upper bound on the primordial value of $n_{6}{ }_{\mathrm{Li}} / n_{7 \mathrm{Li}}:\left(n_{6} \mathrm{Li} / n_{7 \mathrm{Li}}\right)^{\text {obs }} \leq$ $\left(n_{6} \mathrm{Li} / n_{7} \mathrm{Li}\right)^{\text {halo }}$. In our statistical analysis, we use the ratio $n_{6} \mathrm{Li} / n_{\mathrm{H}}$ in deriving the constraints since, with the long-lived exotic particle $X$, this ratio can be calculated more reliably than $n_{6 \mathrm{Li}} / n_{7 \mathrm{Li}}$. In particular, combining Eq. (2.5) with Eq. (2.4), we use the upper bound \#4

$$
\left(n_{6 \mathrm{Li}} / n_{\mathrm{H}}\right)^{\text {obs }} \leq\left(n_{6}{ }_{\mathrm{Li}} / n_{\mathrm{H}}\right)^{\text {halo }}=\left(1.10_{-0.92}^{+5.00}\right) \times 10^{-11}(2 \sigma) .
$$

For the constraint on ${ }^{3} \mathrm{He}$, we adopt the observational ${ }^{3} \mathrm{He}$ abundance of the pre-solar measurements. In this paper, we do not rely upon any detailed models of galactic and stellar chemical evolution because there are large uncertainties in extrapolating back to the primordial abundance. According to such theories of the chemical evolution, the ${ }^{3} \mathrm{He}$ abundance can decrease or increase after the BBN epoch. Therefore, "He-to-H ratio" in itself can not be solely used for a constraint. Instead we adopt the present ratio of ${ }^{3} \mathrm{He}$ to $\mathrm{D}, r_{3,2}$, as the upper bound on the primordial value. This is based on the following simple argument of the chemical evolution. Suppose that some astrophysical process destroys D and/or ${ }^{3} \mathrm{He}$ as

$$
\begin{aligned}
\Delta n^{3} \mathrm{He} & =-R_{3} n^{3} \mathrm{He}, \\
\Delta n_{\mathrm{D}} & =-R_{2} n_{\mathrm{D}},
\end{aligned}
$$

${ }^{\# 3}$ Recently, Suzuki and Inoue 42 pointed out other possibility of producing ${ }^{6} \mathrm{Li}$ independently of the abundance of ${ }^{9} \mathrm{Be}$ through $\alpha-\alpha$ reactions induced by cosmic-ray $\alpha$ accelerated in structure formation shocks. However, it would be difficult to precisely predict the abundance of ${ }^{6} \mathrm{Li}$ in the current version of their model. Therefore, it is premature to quantitatively discuss the abundance by using their model.

${ }^{\# 4}$ Recently, using the Subaru Telescope, ${ }^{6} \mathrm{Li} /{ }^{7} \mathrm{Li}$ was measured in the metal poor subgiant HD 140283 with the use of a high-S/N and high-resolution spectrum: $\left(n_{6 \mathrm{Li}} / n_{\mathrm{H}}\right)^{\text {halo }}<5.0 \times 10^{-12}(2 \sigma)$ [44. The subgiant HD 140283 is the metal-poorest among all of the objects which have been used to derive the bound on ${ }^{6} \mathrm{Li} /{ }^{7} \mathrm{Li}([\mathrm{Fe} / \mathrm{H}]=-2.5)$. It is, however, premature and beyond the scope of this paper to judge its reliability. In addition, our purpose is to obtain a conservative constraints. Thus, we do not use this constraint. 
where $\Delta n_{3} \mathrm{He}\left(\Delta n_{\mathrm{D}}\right)$ is the change of the ${ }^{3} \mathrm{He}(\mathrm{D})$ abundance and $R_{3}\left(R_{2}\right)$ is the probability of destruction of ${ }^{3} \mathrm{He}(\mathrm{D})$. Then, the change of the ratio $r_{3,2} \equiv n^{3} \mathrm{He} / n_{\mathrm{D}}$ is

$$
\Delta r_{3,2} \equiv \frac{n_{3} \mathrm{He}}{n_{\mathrm{D}}+\Delta n_{3} \mathrm{He}}-\frac{n_{3} \mathrm{He}}{n_{\mathrm{D}}}=\frac{R_{2}-R_{3}}{1-R_{2}} r_{3,2}
$$

Since D is more easily destroyed than ${ }^{3} \mathrm{He},{ }^{\# 5}$ it is quite reasonable to assume

$$
R_{2} \geq R_{3}
$$

which leads to $\Delta r_{3,2} \geq 0$. Thus, the ratio $r_{3,2}$ is monotonically increasing function of the cosmic time. Note that, in order to derive this result, we only rely on the fact that $D$ is more fragile than ${ }^{3} \mathrm{He}$. Therefore the present ratio gives us an upper bound on the primordial value of $r_{3,2}$. When we adopt the solar-system data [45], the ${ }^{3} \mathrm{He}$ to $\mathrm{D}$ ratio is given by

$$
r_{3,2}^{\odot}=0.59 \pm 0.54(2 \sigma) .
$$

We take this to be an upper bound on the primordial ${ }^{3} \mathrm{He}$ to $\mathrm{D}$ ratio

$$
r_{3,2}^{\mathrm{obs}} \leq r_{3,2}^{\odot}
$$

Naively it means the upper bound $r_{3,2}^{\text {obs }} \leq 1.13(2 \sigma)$.

\subsection{Current status of SBBN}

In this subsection, we briefly discuss the current status of the SBBN. In the recent years, there have been great progresses in the experiments of the low energy cross sections for 86 charged-particle reactions by the NACRE collaboration [46]. In the compilation, 22 reactions are relevant to the primordial nucleosynthesis, and the old data were revised. In particular, 7 reactions of them are important for the most elementary processes generating nuclei with atomic number up to 7 . Cyburt, Fields and Olive reanalyzed the NACRE data [47, 48. They properly derived the $1 \sigma$ uncertainty and the normalization of the center value for each reaction. In addition, they also reanalyzed the four remaining reactions, using the existing data [49, 50, 51] and the theoretical prediction (for one reaction) [52]. Their efforts are quite useful for the study of the Monte Carlo simulation in BBN, and it was shown that their treatment is consistent with the other earlier studies adopting the results of NACRE [53, 54].

When we perform the Monte Carlo simulation, we adopt the theoretical errors and the center values for 11 elementary nuclear reactions in Refs. [47, 48]. (For the neutron lifetime, see Eq. (5.6).) To systematically take into account the uncertainties, we perform the $\chi^{2}$ fitting including both the observational and theoretical errors which are obtained in Monte Carlo simulation. (See the Appendix in [12.) In our analysis, we assume that the

\footnotetext{
${ }^{\# 5}$ The binding energy of $\mathrm{D}$ is $2.2 \mathrm{MeV}$ while the threshold energies of any destruction processes of ${ }^{3} \mathrm{He}$ are larger.
} 


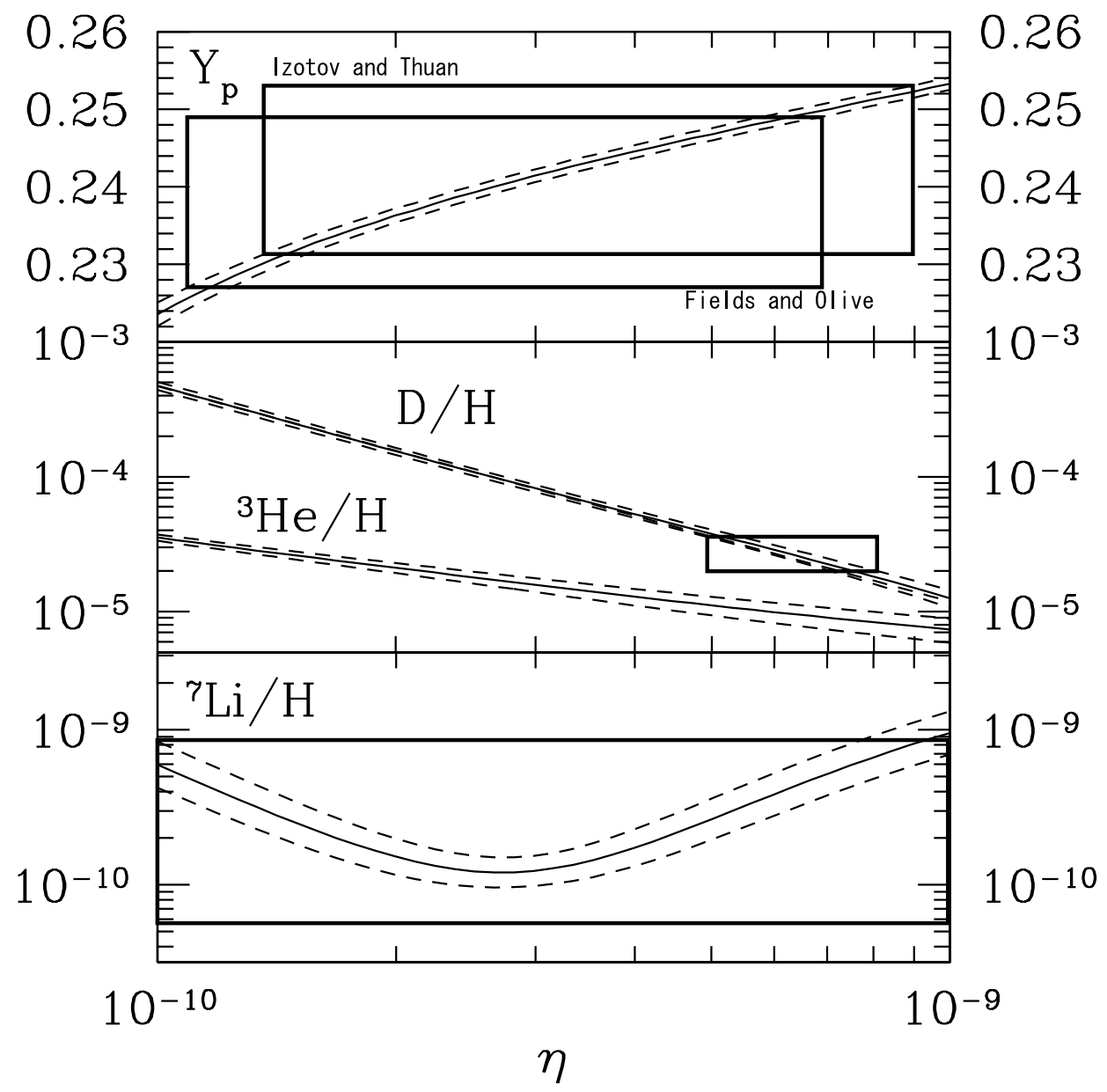

Figure 1: Abundances of the light elements as functions of $\eta$. The solid lines are the center value while the dotted lines show the theoretical uncertainties. Observational constraints are also shown.

theoretical predictions of $n_{\mathrm{D}} / n_{\mathrm{H}}, Y$, and $\log _{10}\left[\left(n_{7_{\mathrm{Li}}} / n_{\mathrm{H}}\right)\right]$ obey the Gaussian probability distribution functions with the widths given by the $1 \sigma$ errors. Concerning the observational values, they are also assumed to obey the Gaussian probability distribution functions. Note that we consider two cases for $Y^{\text {obs }}$, i.e., Fields and Olive (FO) given in Eq.(2.2) and Izotov and Thuan (IT) given in Eq.(2.3).

We calculated the abundances of the light elements as functions of baryon-to-photon ratio:

$$
\eta \equiv \frac{n_{B}}{n_{\gamma}},
$$

where $n_{B}$ and $n_{\gamma}$ are number densities of the baryon and photon, respectively. The results are plotted in Fig. 1. As one can see, theoretical predictions become more or less consistent with the observational constraints when $\eta \sim 6 \times 10^{-10}$.

For a more precise determination of $\eta$, we calculate the $\chi^{2}$-variable as a function of $\eta$, 


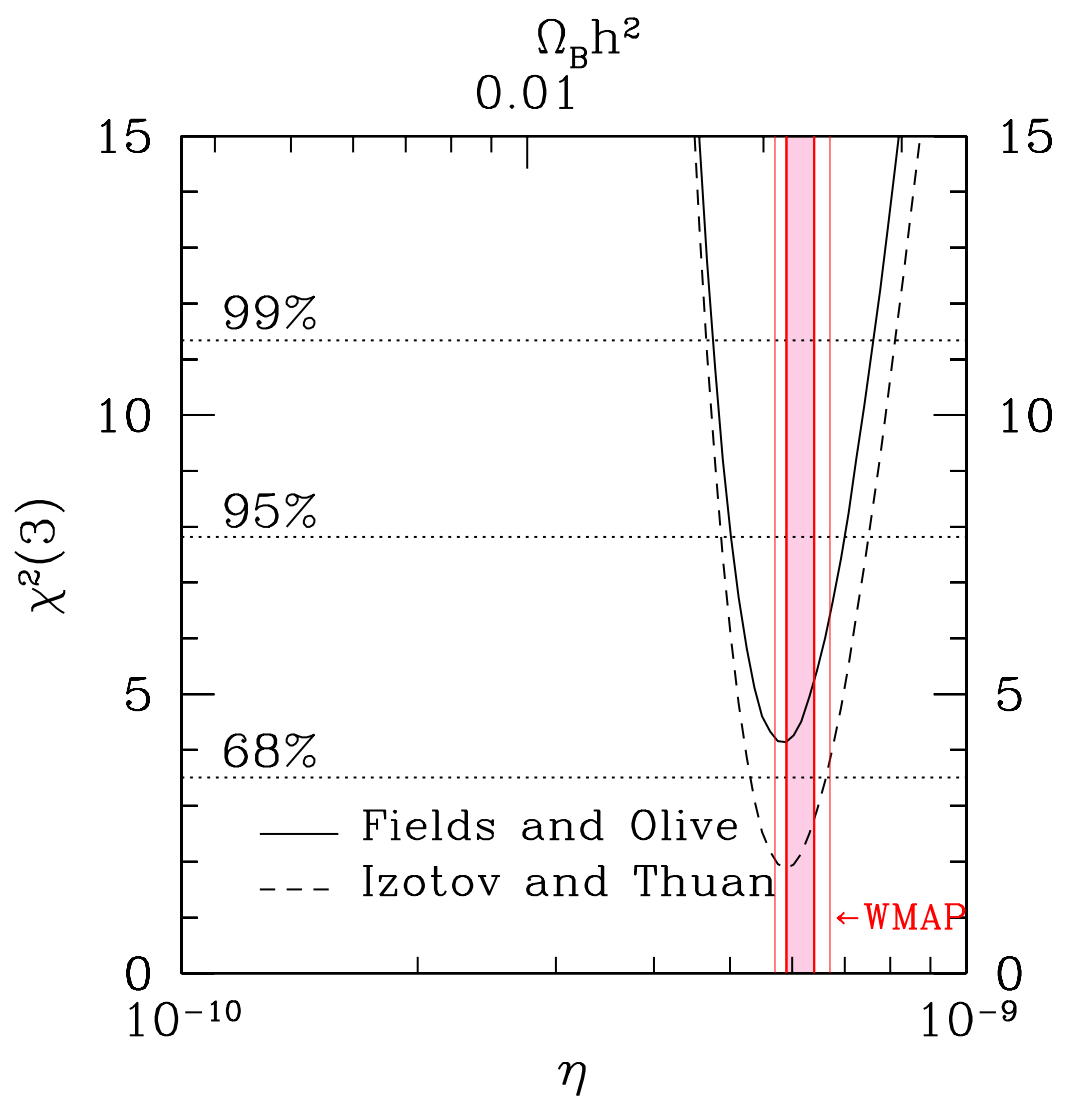

Figure 2: $\chi^{2}$ variable as a function of $\eta$ for SBBN with three degrees of freedom. For the constraint on $Y$, we used Fields and Olive's result (solid) and Izotov and Thuan's (dashed), which are given in Eqs. (2.2) and (2.3), respectively. The shaded band (vertical solid lines) shows the baryon-to-photon ratio suggested by the WMAP at the $1 \sigma(2 \sigma)$ level.

and the result is shown in Fig. 2. The solid line (dashed line) is for the case of Fields and Olive (Izotov and Thuan). From this figure, we see that the prediction of the SBBN agrees with the observation of ${ }^{4} \mathrm{He}, \mathrm{D}$, and ${ }^{7} \mathrm{Li}$ at $95 \%$ C.L. In addition, we obtain the baryon to photon ratio at $95 \%$ C.L. as $\eta^{(\mathrm{SBBN})}=5.85_{-0.85}^{+1.15} \times 10^{-10}\left(5.90_{-1.02}^{+1.63} \times 10^{-10}\right)$ using the value of $Y$ in Fields and Olive (Izotov and Thuan). Since the baryon to photon ratio is related to the baryon density parameter as $\Omega_{B} h^{2}=3.67 \times 10^{7} \eta$, we obtain, at $95 \%$ C.L.,

$$
\Omega_{B} h^{2}=\left\{\begin{array}{ll}
0.0212_{-0}^{+0.00031} & \text { (Fields and Olive) } \\
0.0214_{-0.0037}^{+0.0059} & \text { (Izotov and Thuan) }
\end{array},\right.
$$

where $h$ is Hubble parameter in units of $100 \mathrm{~km} / \mathrm{sec} / \mathrm{Mpc}$.

We also plot the value of $\eta$ reported by the WMAP collaborations in observations of the cosmic microwave background (CMB) anisotropies [21], which is approximately given by

$$
\eta=(6.1 \pm 0.3) \times 10^{-10}
$$


where we have adopted the slightly larger error for the lower bound. The shadowed band in Fig. 2 represents the baryon to photon ratio at $1 \sigma$. The vertical solid lines are their constraints at $2 \sigma$. From this figure, we find that SBBN is consistent with the CMB observation.

Under these circumstances, comparing the predictions of the BBN computations with observations, we can constrain the non-standard scenario such as the radiative decay or the hadronic decay of long-lived massive particles.

Here we should mention that the consistency between theoretical predictions and observed abundances in SBBN or between $\mathrm{CMB}$ and SBBN is partly because we have adopted rather large systematic errors for the observed abundances of ${ }^{4} \mathrm{He}$ and ${ }^{7} \mathrm{Li}$. In fact, if we adopted smaller systematic errors reported in the original papers, we would be confronted with difficulty that the $\eta$ inferred from $\mathrm{D}$ and CMB disagree with that from ${ }^{4} \mathrm{He}$ and ${ }^{7} \mathrm{Li}$ (e.g. see, Ref. [55]). But here we assume large systematic errors and that $\mathrm{SBBN}$ is consistent because the purpose of the present paper is to derive conservative constraints on the massive particles with hadronic decay mode.

\section{Overview}

Before going into the detailed discussion of the BBN with long-lived particle $X$, we give an overview of the cosmological scenario we consider, and define parameters which are used in our analysis.

\subsection{Production}

In this paper, we consider a scenario where a massive particle $X$, with mass $m_{X}$ and decay rate $\Gamma_{X}$, has non-vanishing number density at the early universe. First, we consider the production of $X$ in the early universe. Throughout this paper, we consider a situation where the $X$ particle is somehow produced in the early universe. Production mechanism depends on the property of $X$. For example, if $X$ is a particle, like gravitino, it can be produced by scattering processes of the thermal particles. In addition, it may be also produced by the decay of other particles. Moreover, condensation of some (exotic) scalar field may play the role of $X$. In such a case, non-vanishing initial amplitude of the scalar field provides non-vanishing number density of $X$ at the late stage of the evolution of the universe.

In order to perform our analysis as model-independent as possible, we do not specify the production mechanism of $X$. Indeed, constraints we will obtain depends only on the relic density of $X$ (before it decays). In order to parameterize the number density of $X$ in the early universe, we define the "yield variable"

$$
Y_{X} \equiv \frac{n_{X}}{s}
$$


which is defined at the time $t \ll \Gamma_{X}^{-1}$. Here, $n_{X}$ is the number density of $X$ while $s$ is the total entropy density of the universe. Notice that, as far as we can neglect the entropy production, $Y_{X}$ is a constant when $t \ll \Gamma_{X}^{-1}$.

\subsection{Decay}

In this subsection we discuss the decay of massive particles and its cosmological effects. The overview is schematically presented in Fig. 3 ,

\subsubsection{Decay processes}

In studying the effects of $X$ on the BBN, the decay process of $X$ is classified into two categories; radiative and hadronic decays. These decay processes cause different types of reactions and it is necessary to take account of both processes. Branching ratios decaying into radiative and hadronic particles depend on the properties of $X$. Thus, in order to perform our analysis as model-independent as possible, we define the "hadronic branching ratio"

$$
B_{h}=\frac{\Gamma_{X \rightarrow \text { hadrons }}}{\Gamma_{X}}
$$

where $\Gamma_{X}$ is the decay rate of $X$ and $\Gamma_{X \rightarrow \text { hadrons }}$ is the hadronic decay width of $X$.

If $X$ may directly decay into hadronic particles, $B_{h}$ may become close to 1 . In addition, even when $X$ primarily decays into photon (and other non-hadronic particles), $B_{h}$ is expected to be non-vanishing since the quark-anti-quark pair can be attached at the end of the (virtual) photon line.

For example, for the case where the unstable gravitino $\psi_{\mu}$ plays the role of $X$, which is one of the most well-motivated cases, a gravitino may directly decay into gluon-gluino and/or quark-squark pairs. (See Fig. 4) If the decay rate of these modes are sizable, $B_{h}$ becomes close to 1 . If these hadronic decay modes are kinematically blocked, however, the gravitino may primarily decay into photon and the neutralino $\tilde{\chi}^{0}: \psi_{\mu} \rightarrow \gamma+\tilde{\chi}^{0}$. If this is the only kinematically allowed two-body decay mode of the gravitino, $B_{h}$ becomes much smaller than 1. However, even in this case, it is also expected that $B_{h}$ is non-vanishing since the decay mode $\psi_{\mu} \rightarrow q+\bar{q}+\tilde{\gamma}$ (with $q$ and $\bar{q}$ being the quark and anti-quark, respectively) exists. (See Fig. [5]) In this case, $B_{h}$ is expected to be $B_{h} \sim 10^{-(2-3)}$.

In our analysis, we also introduce several parameters in order to characterize the decay of $X$. First, we assume that each primary parton jet has the energy $E_{\text {jet }}$. (For example, when $X$ decays into $q-\bar{q}$ pair, $E_{\text {jet }}=\frac{1}{2} m_{X}$.) This parameter is used when we study the hadronization processes with JETSET event generator. On the contrary, for the decay process of $X$ with energetic photon in the final state, we define $E_{\gamma}^{(0)}$ which is the energy of the emitted photon. In addition, in some case, invisible particle may be also emitted. For example, when the gravitino plays the role of $X$, some fraction of the energy is carried away by the LSP which we assume is the neutralino. Thus, we define $E_{\text {vis }}$, which is the 


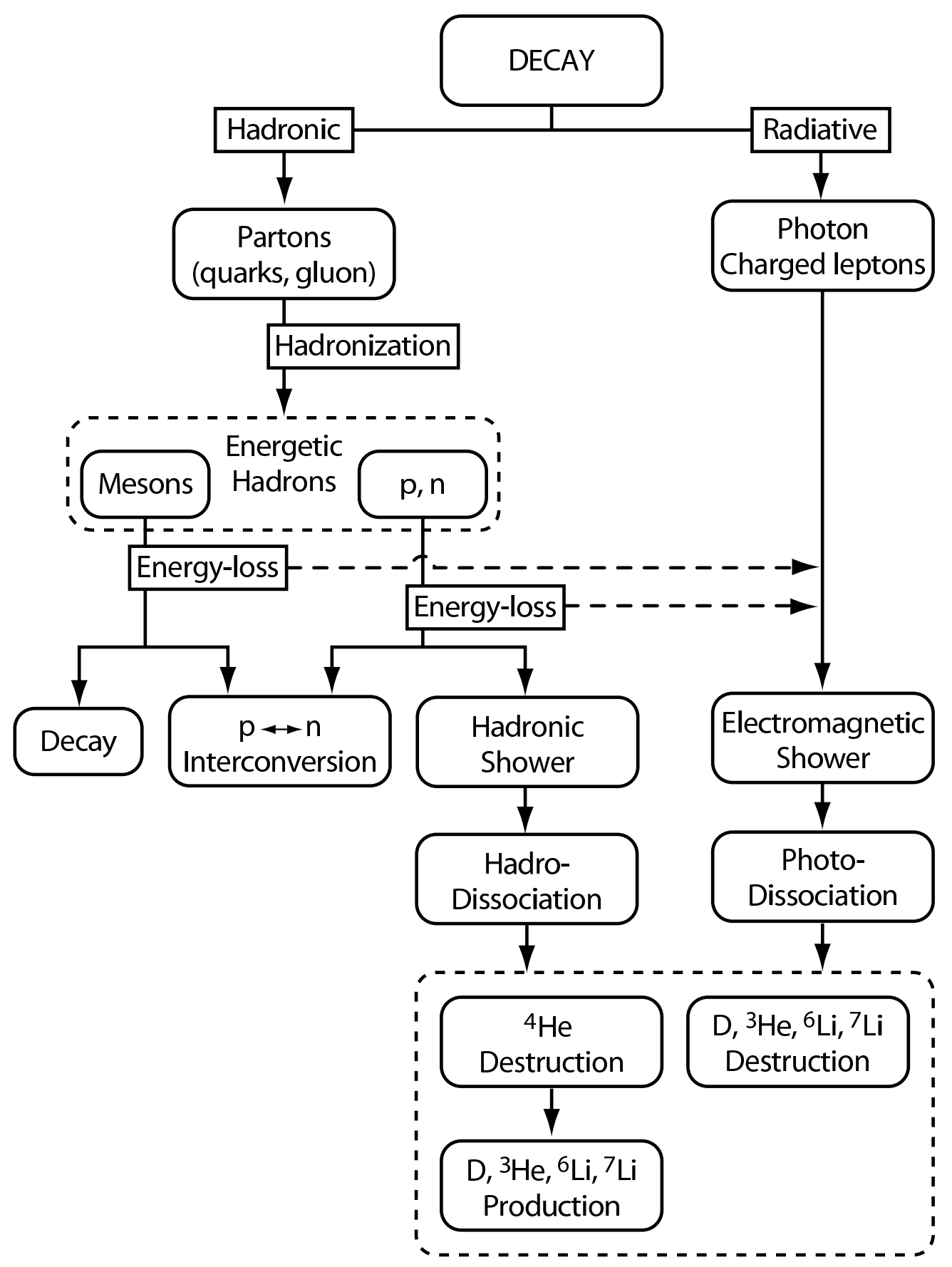

Figure 3: Flow-chart of the hadronic decay of massive particles. 

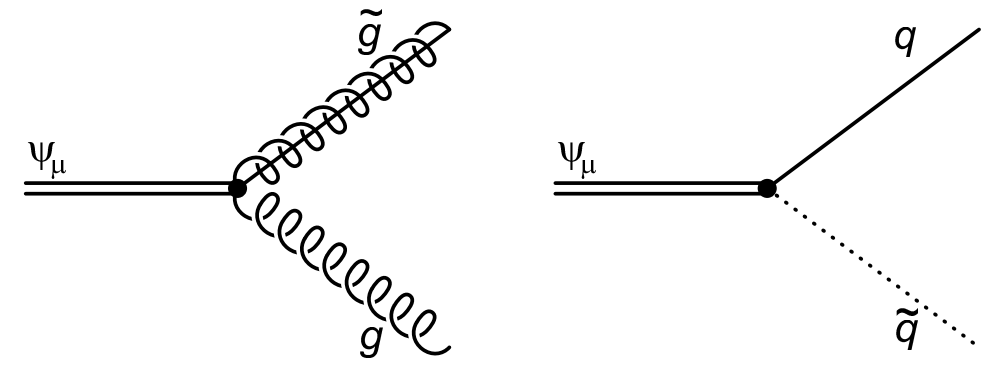

Figure 4: Feynman diagrams for the decay processes $\psi_{\mu} \rightarrow g+\tilde{g}$ and $\psi_{\mu} \rightarrow q+\tilde{q}$, where $\psi_{\mu}$, $g, \tilde{g}, q$, and $\tilde{q}$ are the gravitino, gluon, gluino, quark, and squark, respectively. Here, the black blob represents the vertex originating from the gravitino-supercurrent interaction.
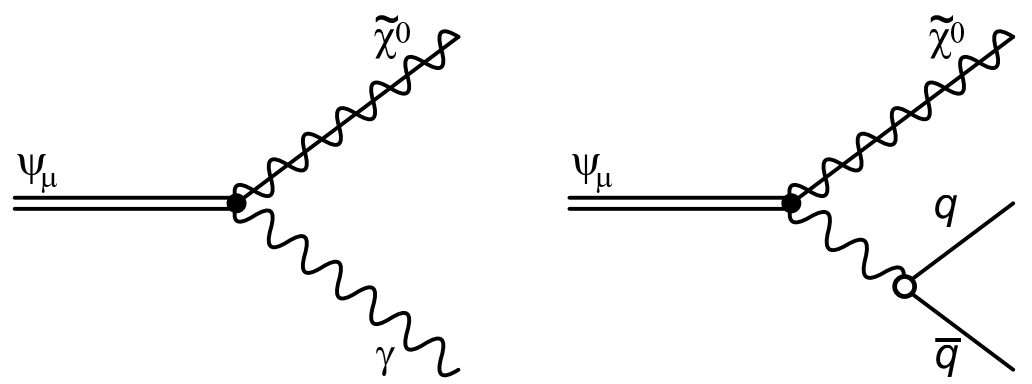

Figure 5: Same as Fig. (4 but for the radiative decay modes. (Here, $\gamma$ is the photon while $\tilde{\chi}^{0}$ is the neutralino.) 
(averaged) energy emitted in the form of the "visible" particles. As we will discuss, number of high-energy photons produced in the electromagnetic cascade process is proportional to $E_{\text {vis }}$.

\subsubsection{Radiative decay}

In the radiative decay the massive particles decay into photons (or electrons). The emitted photons (electrons) have energies of the order of the mass of the parent particles, $m_{X} \sim$ $\mathcal{O}(100) \mathrm{GeV}$. Such high energy photons (electrons) scatter off the background photons and induce electromagnetic showers in which high energy photons and electrons are copiously produced through electromagnetic interactions with the background cosmic plasma (see the right branch in Fig. 31). Some of soft photons produced secondarily in the shower destroy the light elements $\left({ }^{4} \mathrm{He},{ }^{3} \mathrm{He}, \mathrm{D},{ }^{7} \mathrm{Li}\right.$ and $\left.{ }^{6} \mathrm{Li}\right)$ synthesized in BBN before they lose energy mainly through Thomson scattering (photodissociation). This photodissociation process not only destroys light element but also produce ${ }^{3} \mathrm{He}, \mathrm{D}$, and ${ }^{6} \mathrm{Li}$ as result of the destruction of ${ }^{4} \mathrm{He}$. Since the number density of ${ }^{4} \mathrm{He}$ is much larger than the other light elements, the destruction of tiny fraction of the ${ }^{4} \mathrm{He}$ leads to significant production of ${ }^{3} \mathrm{He}$, $\mathrm{D}$, and ${ }^{6} \mathrm{Li}$.

In the case of radiative decay, the most important process that determines the destruction rate of the light elements is "photon-photon process" where the high energy photons scatter off the background photons into electrons and positrons. Since the number of the background photons is about $10^{10}$ times larger than that of electrons, the photon-photon process, if it occurs, quickly thermalizes the high energy photons and the destruction of light elements does not take place frequently. However, since there exists the threshold of the photon energy $\left(E_{t h} \sim m_{e} / 22 T\right)$, soft photons with energy less than the threshold can destroy the light elements. When the background temperature is so high $(T \gtrsim 0.01 \mathrm{MeV})$, $E_{t h}$ is smaller than the binding energy of D which is the most fragile, and all light elements survive. As the cosmic temperature decreases, $E_{t h}$ becomes larger. Then, the soft photons can destroy $\mathrm{D}$ at $T \lesssim 0.01 \mathrm{MeV},{ }^{3} \mathrm{He},{ }^{7} \mathrm{Li}$ at $T \lesssim 0.003 \mathrm{MeV}$, and ${ }^{4} \mathrm{He}$ at $T \lesssim 0.001 \mathrm{MeV}$. Therefore, roughly speaking, the constraint on the radiatively decaying particle comes from the destruction of D for lifetime less than $10^{6} \mathrm{sec}$ and from the overproduction of D and ${ }^{3} \mathrm{He}$ due to the destruction of ${ }^{4} \mathrm{He}$ for lifetime longer than $10^{6} \mathrm{sec}$.

\subsubsection{Hadronic decay}

When quarks or gluons are emitted in the decay of the massive particles, they firstly fragment into a lot of hadrons and form hadron jets. As a result, many high energy mesons and nucleons are injected into the cosmic plasma.

At earlier epochs $(t \lesssim 100 \mathrm{sec})$ the high energy mesons and nucleons lose their energy very quickly through electromagnetic interaction. Thus, they are completely stopped and reach to the kinetic equilibrium. Thus, the emitted hadrons do not directly destroy the light elements. After energy loss, they scatter off the background $p$ or $n$ through the strong interaction with their threshold cross sections. Then, they inter-convert the background 
$p$ and $n$ each other, even after the normal freeze-out time of the $n / p$ ratio of the weak interaction. Since the typical mean free time for strong interaction is $\mathcal{O}\left(10^{-8}\right)$ sec, only mesons with relatively long lifetimes such as $\pi^{ \pm}$and $K^{0, \pm}$ and nucleons $(p, n, \bar{p}, \bar{n})$ can cause the $p$ - $n$ inter-conversion. Since, at $T \lesssim 1 \mathrm{MeV}$, the proton is more abundant than the neutron, the conversion from $p$ to $n$ takes place more frequently than its inverse process, and hence the hadron injection extraordinarily tends to increase the ratio $n / p$. As a result, the produced ${ }^{4} \mathrm{He}$ and $\mathrm{D}$ would increase in the hadron injection scenario compared to the SBBN case.

At later epoch $(t \gtrsim 100 \mathrm{sec})$, mesons decay before they interact the background nucleons, and hence they become cosmologically irrelevant. On the other hand the emitted high energy protons and neutrons can scatter off the background $p, n$ and ${ }^{4} \mathrm{He}$ (which is synthesized in BBN). Since the energy loss due to the electromagnetic interaction is insufficient, the high energy $p$ and $n$ interact with background hadrons before they lose energy, and produce secondary hadrons through elastic and inelastic collisions. Such hadronic interactions occur successively and evolve hadronic showers. During evolution of the hadronic shower, a lot of ${ }^{4} \mathrm{He}$ 's are destroyed by the inelastic collisions, and $\mathrm{D}, \mathrm{T}$ and ${ }^{3} \mathrm{He}$ are produced from the ${ }^{4} \mathrm{He}$ dissociation. Then the energetic $\mathrm{T}$ and ${ }^{3} \mathrm{He}$ scatter off the background ${ }^{4} \mathrm{He}$, and produce ${ }^{6} \mathrm{Li}$ and ${ }^{7} \mathrm{Li}$. Since ${ }^{4} \mathrm{He}$ is much more abundant than the other light elements, those non-thermal production of $\mathrm{D},{ }^{3} \mathrm{He},{ }^{6} \mathrm{Li}$ and ${ }^{7} \mathrm{Li}$ drastically changes the prediction of SBBN. Thus, the severe constraint is imposed on the hadronic decay. Contrary to the radiative decay, non-thermal production due to ${ }^{4} \mathrm{He}$ dissociation is even important at $t \lesssim 10^{6}$ sec when the high energy photons quickly lose their energy by the photon-photon process and cannot destroy ${ }^{4} \mathrm{He}$.

Finally, we remark that the almost all of the energy of the primary hadrons are transferred to the electrons (positrons) and photons through the electromagnetic energy loss processes, and decays of mesons and heavy charged leptons. Then the energetic photons and electrons cause electromagnetic showers. In this sense, the hadronic decay also has the same effect as the radiative decay. This is indicated by dashed arrows in Fig. 3.

\section{Photodissociation}

We are at the position to discuss various processes induced by the hadronic (as well as the radiative) decay of the late-decaying particle $X$. In calculating of the abundances of the light elements, we take account of two types of dissociation processes of the light elements; one is the photodissociations induced by the energetic photons and the other is the hadrodissociations by the hadrons. Importantly, even if we consider hadronic decay modes of $X$, kinetic energy of the hadrons are eventually converted to radiation via the scattering processes. Thus, even in the case of the hadronic decay mode, it is important to consider the photodissociation processes. In this section, we first discuss the simple photodissociation reactions of the light elements.

Once the late-decaying particle $X$ decays in the thermal bath, most of the (visible) 
energy released by the decay of $X$ is eventually converted to the form of the photon for the situation we are interested in. Then, the electromagnetic cascade processes are induced. In order to study the photodissociation processes of the light elements, it is necessary to understand the spectrum of the high energy photon generated by the cascade process. In our study, we have calculated the photon spectrum taking account of effects of the following processes:

- Injection of the high energy photon from the radiative decay of $X$

- Double photon pair creation $\left(\gamma+\gamma_{\mathrm{BG}} \rightarrow e^{+}+e^{-}\right)$

- Photon-photon scattering $\left(\gamma+\gamma_{\mathrm{BG}} \rightarrow \gamma+\gamma\right)$

- Compton scattering off thermal electron $\left(\gamma+e_{\mathrm{BG}}^{-} \rightarrow \gamma+e^{-}\right)$

- Inverse Compton scattering off background photon $\left(e^{ \pm}+\gamma_{\mathrm{BG}} \rightarrow e^{ \pm}+\gamma\right)$

- Pair creation in background proton (and $\left.\alpha_{\mathrm{BG}}\right)\left(\gamma+p_{\mathrm{BG}} \rightarrow e^{+}+e^{-}+p\right)$

For details of the calculation of the photon spectrum, see Appendix A

One important point is that the energy distribution of the photon in the electromagnetic shower is mostly determined by the total amount of the injected energy and is insensitive to the detail of the primary spectrum of the injected high-energy particles. Thus, the photon spectrum depends on the temperature, number density of $X$, decay rate of $X$, and the total visible energy released by the single decay of $X$ which we call $E_{\text {vis }}$; once these parameters are fixed, the photon spectrum $f_{\gamma}$ is determined.

In addition, if the temperature is high enough, pairs of charged particles like $\mu^{+} \mu^{-}$, $\pi^{+} \pi^{-}$, and so on, may be produced by the photon-photon scattering. Such pair-production processes, however, do not significantly change the photodissociation rates since photon spectrum for the photon energy relevant for the photodissociation processes is determined by the Compton scattering and the $e^{+} e^{-}$pair creation in the nuclei. Thus, for the study of the photodissociation, we can neglect the pair production of charged particles heavier than the electron. If the hadronic particles are pair-produced, however, it may provide new sources of the hadronic particles. Such effects will be considered in the next section.

Effects of the photodissociation is taken into account by including the following terms into the Boltzmann equations describing the evolutions of the light elements:

$$
\begin{aligned}
{\left[\frac{d n_{A_{i}}}{d t}\right]_{\text {photodiss }}=} & -n_{A_{i}} \sum_{j} \int_{E_{\gamma}^{(\mathrm{th})}} d E_{\gamma} \sigma_{A_{i} \rightarrow A_{j}}\left(E_{\gamma}\right) f_{\gamma}\left(E_{\gamma}\right) \\
& +\sum_{j} n_{A_{j}} \int_{E_{\gamma}^{(\mathrm{th})}} d E_{\gamma} \sigma_{A_{j} \rightarrow A_{i}}\left(E_{\gamma}\right) f_{\gamma}\left(E_{\gamma}\right),
\end{aligned}
$$

where $\sigma_{A_{i} \rightarrow A_{j}}$ is the cross section for the process $A_{i}+\gamma \rightarrow A_{j}+\cdots$ with the threshold energy $E_{\gamma}^{(\text {th })}$, and the summations are over all the possible reactions. (In this paper, $A_{i}$ is 


\begin{tabular}{lll}
\hline \hline Reaction & Error & Reference \\
\hline$\gamma+\mathrm{D} \rightarrow n+p$ & $6 \%$ & {$[56]$} \\
$\gamma+\mathrm{T} \rightarrow n+\mathrm{D}$ & $14 \%$ & {$[57,[58]$} \\
$\gamma+\mathrm{T} \rightarrow p+n+n$ & $7 \%$ & {$[58]$} \\
$\gamma+{ }^{3} \mathrm{He} \rightarrow p+\mathrm{D}$ & $10 \%$ & {$[59]$} \\
$\gamma+{ }^{3} \mathrm{He} \rightarrow p+p+n$ & $15 \%$ & {$[59]$} \\
$\gamma+{ }^{4} \mathrm{He} \rightarrow p+\mathrm{T}$ & $4 \%$ & {$[60]$} \\
$\gamma+{ }^{4} \mathrm{He} \rightarrow n+{ }^{3} \mathrm{He}$ & $5 \%$ & {$[61,62]$} \\
$\gamma+{ }^{4} \mathrm{He} \rightarrow p+n+\mathrm{D}$ & $14 \%$ & {$[60]$} \\
$\gamma+{ }^{6} \mathrm{Li} \rightarrow$ anything & $4 \%$ & {$[63]$} \\
$\gamma+{ }^{7} \mathrm{Li} \rightarrow n+{ }^{6} \mathrm{Li}$ & $4 \%$ & {$[64$} \\
$\gamma+{ }^{7} \mathrm{Li} \rightarrow$ anything & $9 \%$ & {$[65]$} \\
$\gamma+{ }^{7} \mathrm{Be} \rightarrow p+{ }^{6} \mathrm{Li}$ & & \\
$\gamma+{ }^{7} \mathrm{Be} \rightarrow$ anything & & \\
\hline \hline
\end{tabular}

Table 1: Photodissociation reactions included in our analysis. We also write down the errors of each cross sections which are used in our Monte Carlo analysis.

used for light elements, i.e., $n, p$, D, T, ${ }^{3} \mathrm{He},{ }^{4} \mathrm{He},{ }^{6} \mathrm{Li},{ }^{7} \mathrm{Li}$, and ${ }^{7} \mathrm{Be}$.) The above equation for $i$-th nucleus can be also written as

$$
\left[\frac{d n_{A_{i}}}{d t}\right]_{\text {photodiss }}=-\sum_{j} \Gamma_{A_{i} \rightarrow A_{j}}^{(\text {photodiss })} n_{A_{i}}+\sum_{j} \Gamma_{A_{j} \rightarrow A_{i}}^{(\text {photodiss })} n_{A_{j}},
$$

where

$$
\Gamma_{A_{j} \rightarrow A_{i}}^{\text {(photodiss) }} \equiv \int_{E_{\gamma}^{\text {(th }}} d E_{\gamma} \sigma_{A_{i} \rightarrow A_{j}}\left(E_{\gamma}\right) f_{\gamma}\left(E_{\gamma}\right)
$$

Thus, the BBN reactions with the radiatively decaying particle can be followed once the quantities $\Gamma_{A_{j} \rightarrow A_{i}}^{\text {(photodis) }}$ are known.

In our analysis, we have calculated $\Gamma_{A_{i} \rightarrow A_{j}}^{\text {(photodiss) }}$ for all the relevant processes. In order to derive the constraint on the primordial abundance of $X$, we calculate the abundances of the light elements up to ${ }^{7} \mathrm{Li}$. All the processes included in our analysis are listed in Table 1. For the photodissociation of D, we use the cross section in the analytic form. For the other processes, the cross sections are taken from experimental data. For the photodissociation cross sections of ${ }^{7} \mathrm{Be}$, we could not find experimental data. Thus, we use the photodissociation rates of ${ }^{7} \mathrm{Li}$ for corresponding dissociation processes of ${ }^{7} \mathrm{Be}$; $\Gamma_{7 \mathrm{Be} \rightarrow A}^{\text {(photodiss })} \simeq \Gamma_{7 \mathrm{Li} \rightarrow A}^{\text {(photodiss })}$. We have checked that, in deriving the constraints on the properties of $X,{ }^{7}$ Be does not play a significant role. Thus, our results are not significantly affected by this assumption.

Since the abundance of ${ }^{4} \mathrm{He}$ is large, the photodissociation of ${ }^{4} \mathrm{He}$ may significantly change the abundances of nuclei with atomic number $A \leq 4$. Thus, in considering the 
photodissociation of nuclei with $A \leq 4$, we specify the final-state in order for precise calculation of the abundances of the light elements. The dissociation of Li, on the contrary, do not change the abundances of the nuclei lighter than Li because of the smallness of the abundances of ${ }^{6} \mathrm{Li}$ and ${ }^{7} \mathrm{Li}$. Thus, for most of the photodissociation processes of $\mathrm{Li}$, we do not specify the final-state particles. The only exception is the process ${ }^{7} \mathrm{Li}+\gamma \rightarrow n+{ }^{6} \mathrm{Li}$; this process may be important for the calculation of the ${ }^{6} \mathrm{Li}$ abundance; In our analysis, the effect of the ${ }^{6} \mathrm{Li}$ production through this process is properly taken into account.

So far, we have discussed the scatterings of the photons with the background nuclei. Importantly, since the photons are energetic, the final-state particles produced by the photodissociation processes may acquire sizable energy and participate in other class of non-thermal production processes of the light elements. In particular, energetic $\mathrm{T}$ and ${ }^{3} \mathrm{He}$ may scatter off the background $\alpha$ to produce ${ }^{6} \mathrm{Li}$. Since the observational upper bound on the primordial abundance of ${ }^{6} \mathrm{Li}$ is very stringent, non-thermal production of ${ }^{6} \mathrm{Li}$ may impose significant constraint on the properties of $X$ [13, 14. Such non-thermal production of ${ }^{6} \mathrm{Li}$ will be discussed in Section 8 in detail.

\section{Hadronic Decay of Massive Particle}

Now, we consider hadronic decay of $X$ and its effects on the light-element abundances. For this purpose, it is important to understand how the partons emitted by the decay of $X$ are hadronized and how the hadrons propagate in the thermal bath. Thus, let us discuss these subjects in this section.

\subsection{Hadronization}

We first consider the hadronization processes. Since we mostly consider the cases where the mass of $X$ is larger than the QCD scale, $X$ primarily decays into the quarks and/or gluons for the hadronic decay process. For the cosmic temperature we consider, however, the time scale for the hadronization is much shorter than that for the cosmic expansion. Thus, the partons emitted from the decay of $X$ are instantly hadronized and fragment into the mesons and nuclei $\left(\pi^{ \pm}, \pi^{0}, K^{ \pm}, K_{L, S}^{0}, n, p, \Lambda^{0}\right.$, and so on). In studying the evolution of the cascade processes, which will be discussed in the next section, those energetic nuclei and mesons (in particular, $p$ and $n$ ) become the primary particles which cause the hadronic shower.

In our study, the hadronization processes are followed by using the JETSET 7.4 Monte Carlo event generator [20, which computes the fragmentation of the hadrons for the processes with $q \bar{q}$. Importantly, predictions of the JETSET package well agree with the experimental data. Thus, in our analysis, we expect that the uncertainties arising from the hadronization processes are small enough to be neglected. To demonstrate the agreements of the results from the JETSET package with experiments, in Fig. [6] we plot the averaged charged-particle multiplicity $\left\langle N_{\mathrm{ch}}\right\rangle$ (which is the total number of the charged hadrons) as 


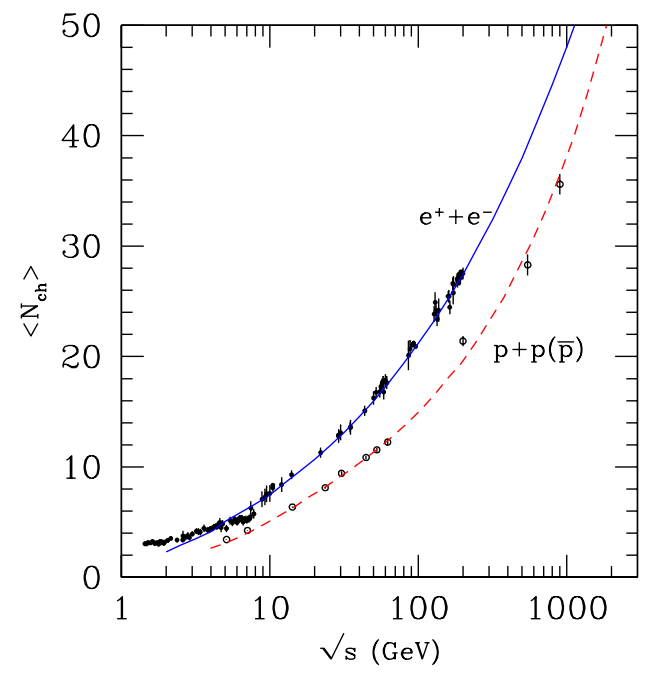

Figure 6: Charged-particle multiplicity $\left\langle N_{\mathrm{ch}}\right\rangle$ per two hadron jets as a function of the center of mass energy $\sqrt{s}$ [66. The solid line is the case with $e^{+} e^{-}$annihilation, while the dashed one is for the case with $p+p(\bar{p})$ collisions. For the $p+p(\bar{p})$ case, we use PYTHIA Monte Carlo event generator and do not include the single diffraction.

a function of the center-of-mass energy of the $q \bar{q}$ system $\sqrt{s}$. $\# 6$ From Fig. 6 we find that the predicted $\left\langle N_{\mathrm{ch}}\right\rangle$ well agrees with the experimental values. In addition, as we can see from the figure, $\left\langle N_{\mathrm{ch}}\right\rangle$ increases as $\sqrt{s}$ increases.

In addition, in Figs. 7 and 8, we plot the spectra of the mesons $\left(\pi^{+}+\pi^{-}, K^{+}+K^{-}\right.$, and $\left.K_{L}^{0}\right)$ and baryons $(n+\bar{n}$, and $p+\bar{p})$, respectively, as functions of their kinetic energy in the center-of-mass frame $E_{\text {kin }}^{(\mathrm{CM})}$. Here we take the total energy of the jets to be $\sqrt{s}=91.2$ $\mathrm{GeV}$, for which we see that the typical energy of the produced hadrons are $\mathcal{O}(10) \mathrm{GeV}$. In studying the effects of the hadronic decay of $X$, we calculate the spectra of the primary hadrons with the relevant total energy of the jets which is determined by the mass of $X$ and the decay mode. For $m_{X} \sim \mathcal{O}(100) \mathrm{GeV}-\mathcal{O}(100) \mathrm{TeV}$, we have found that the typical energy of the produced hadrons is about $\mathcal{O}(1-100) \mathrm{GeV}$

In Fig. 9 we also plot the averaged number of the produced hadron per two hadron jets as a function of the center-of-mass energy $\sqrt{s}$. From this figure, we can see that the hadrons directly emitted from the decay of $X$ is mostly pions. In addition, in Fig. 10 we plot the averaged numbers of the produced hadrons in $p+p(\bar{p})$ collision. We see that the number of the secondarily-produced nucleon-anti-nucleon pairs is small for the center-of-mass relevant for our study $\sqrt{s} \lesssim \mathcal{O}(10) \mathrm{GeV}$. Therefore, we ignore them in this

\footnotetext{
${ }^{\# 6}$ Here $\left\langle N_{\mathrm{ch}}\right\rangle$ is defined as the value after the decay of the $K_{S}$ and $\Lambda^{0}$ since their lifetimes $\left(\tau_{K_{S}^{0}}=\right.$ $0.89 \times 10^{-10}$ sec and $\tau_{\Lambda^{0}}=2.63 \times 10^{-10} \mathrm{sec}$, respectively 66 ) are much shorter than the cosmological time scale we are interested in. For details, see the subsection 5.2
} 


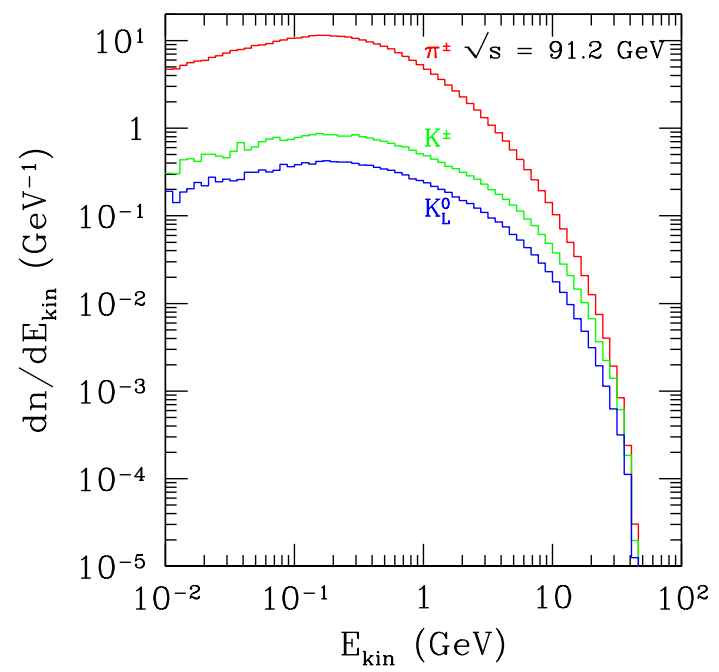

Figure 7: Spectra of the mesons $\left(\pi^{+}+\pi^{-}, K^{+}+K^{-}\right.$, and $\left.K_{L}^{0}\right)$ produced by the $e^{+} e^{-}$ annihilation process as functions of their kinetic energy $E_{\text {kin }}$. The center-of-mass energy is taken to be $\sqrt{s}=91.2 \mathrm{GeV}$.

paper.

In considering the hadronic processes in our analysis, we use the hadron fragmentation obtained for the process $e^{+}+e^{-} \rightarrow \bar{q}+q$ with $\sqrt{s}=2 E_{\text {jet }}$ as a primary spectrum of the hadrons generated from $X$. Here, $E_{\text {jet }}$ is the energy of the primary jet, and will be related to $m_{X}$ later. We have checked that our results are insensitive to the Lorentz and color structure of the $q \bar{q}$ system as far as the value of $\sqrt{s}$ is fixed.

As we mentioned in the previous section, there is another possible production process of hadrons via the scattering of the high energy photons emitted from $X$ with the background photons. In particular, the lightest charged mesons, $\pi^{ \pm}$, can be generated from the process $\gamma+\gamma_{\mathrm{BG}} \rightarrow \pi^{+}+\pi^{-}$. Such mesons may contribute to the inter-conversion processes between the proton and neutron, which will be discussed in Section 6 .

For the high energy photon with energy $E_{\gamma}$, the center-of-mass energy of such process is $\sqrt{s} \sim \sqrt{3 E_{\gamma} T}$. Thus, pair-creation rate of the pion becomes sizable only when the temperature is high enough. Importantly, if the pair creation of the charged pions are effective, electron-positron (and other lepton) pair can be also produced without kinematical suppression. Thus, we estimate the number of the pions produced by the radiative decay of a single $X$ as

$$
\xi_{\pi^{ \pm}}^{(\mathrm{rad})}=\left[\frac{\sigma_{\gamma+\gamma \rightarrow \pi^{+}+\pi^{-}}}{\sum_{l} \sigma_{\gamma+\gamma \rightarrow l^{+}+l^{-}}+\sigma_{\gamma+\gamma \rightarrow \pi^{+}+\pi^{-}}}\right]_{s=3 E_{\gamma}^{(0)} T},
$$

where $\sigma_{\gamma+\gamma \rightarrow \pi^{+}+\pi^{-}}$and $\sigma_{\gamma+\gamma \rightarrow l^{+}+l^{-}}$are cross sections of the pion and charged lepton pair 


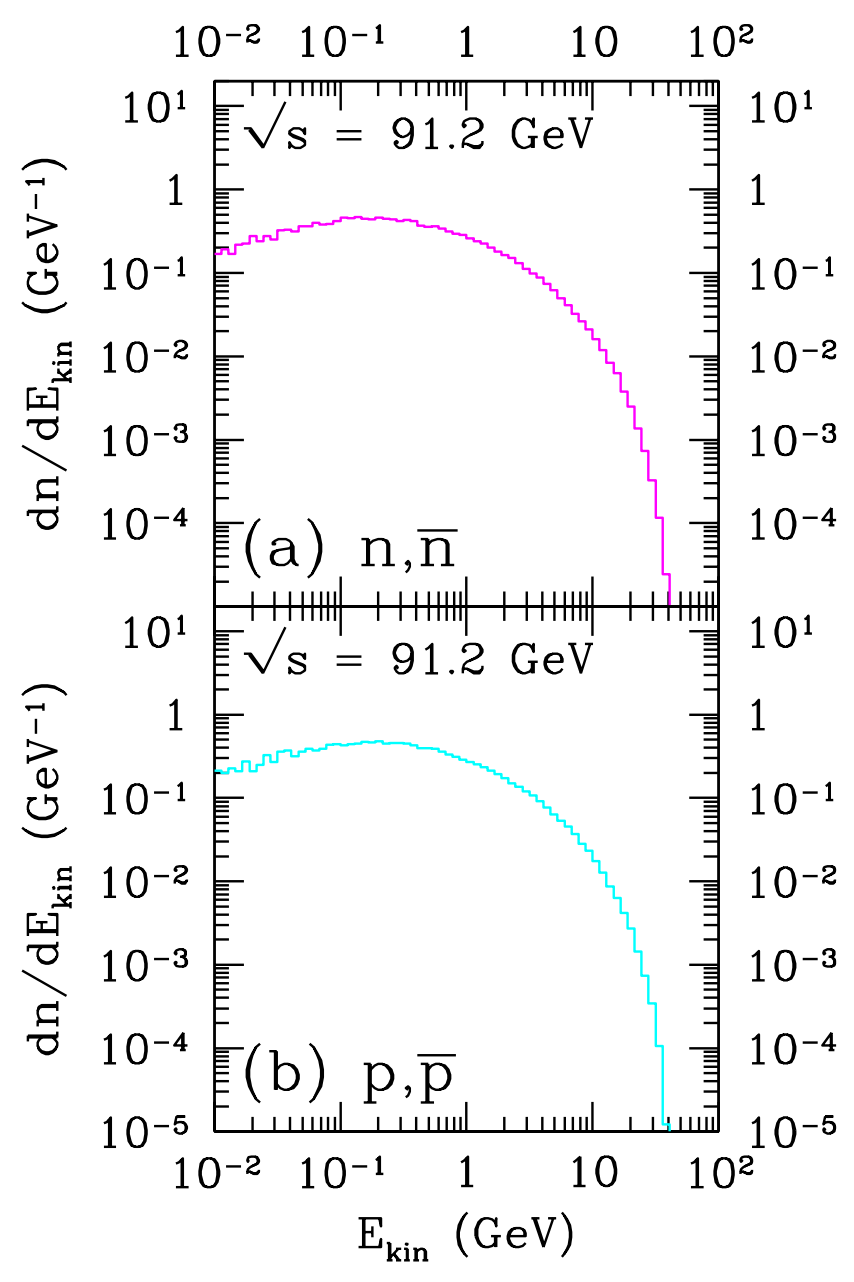

Figure 8: Spectra of the baryons ((a) $n+\bar{n}$ and (b) $p+\bar{p}$ ) produced by the $e^{+} e^{-}$annihilation process as functions of their kinetic energy $E_{\text {kin }}$. The center-of-mass energy is taken to be $\sqrt{s}=91.2 \mathrm{GeV}$. 


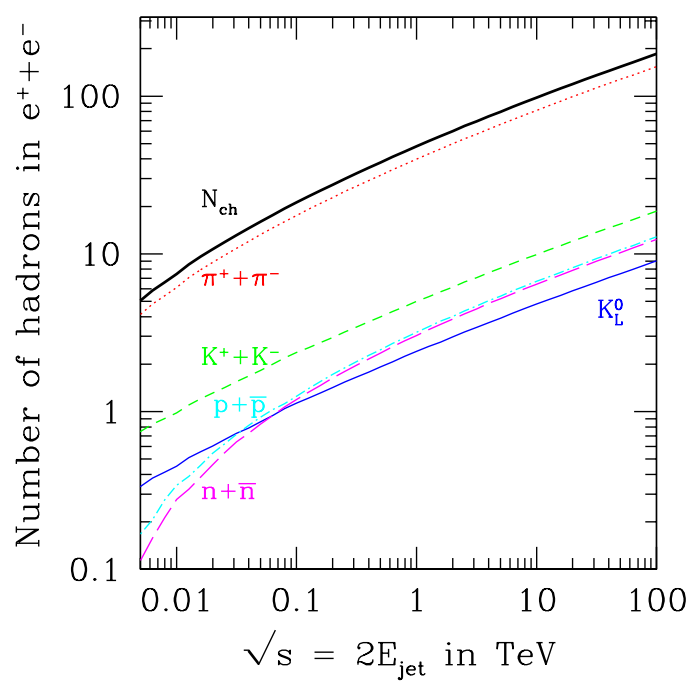

Figure 9: Number of the hadrons produced by the $e^{+} e^{-}$annihilation process as functions of the center-of-mass energy $\sqrt{s}$. The dotted, the short dashed, the thin solid, the dotdashed, and the long dashed lines are $\pi^{+}+\pi^{-}, K^{+}+K^{-}, K_{L}^{0}, p+\bar{p}$, and $n+\bar{n}$, respectively. We also plot the charged-particle multiplicity $N_{\text {ch }}$ by the thick solid line.

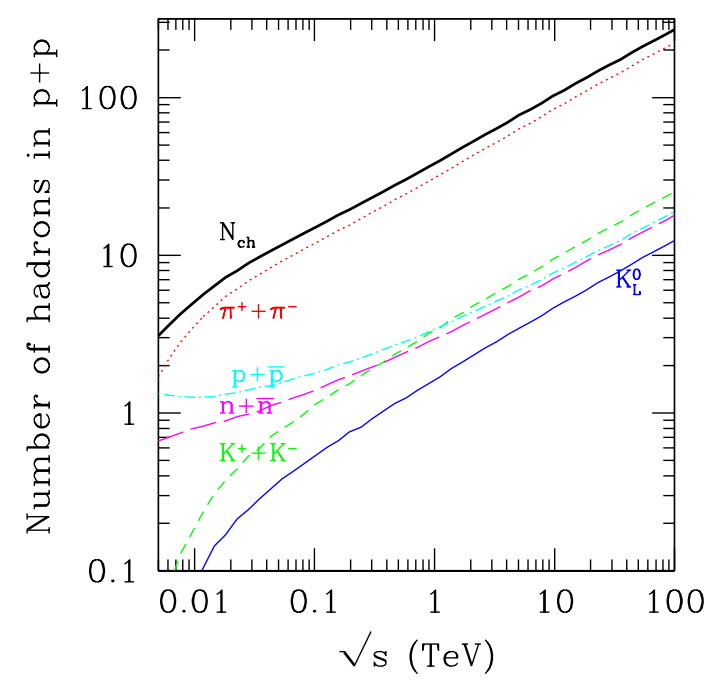

Figure 10: Number of the hadrons produced by the $p+p(\bar{p})$ scattering process. Meanings of the lines are the same as Fig. 9 . 
creation processes, respectively. Those cross sections are evaluated at $s=3 E_{\gamma}^{(0)} T$. For the explicit formulae for these cross sections, see [67, 68. Here, we only take account of the charged pion production by the primary photons directly emitted from the decay of $X$. Since the energy of the photon is drastically reduced after several steps of pair creations (and inverse Compton scattering of electron and positron), the pair-creation processes are mostly induced by the photons directly emitted from the decay process of $X$. In addition, if the energy of the primary photon become large, hadrons heavier than the pions may be produced by the photon-photon scattering processes. However, heavier hadrons mostly decay before inducing the inter-conversion processes. (Exceptions are kaons for some cases. Since the effects of the kaons are not included in discussing the inter-conversion effects, we do not consider the pair creation of the charged kaons here. For details, see Section 6.) In addition, productions of heavier hadrons are kinematically suppressed. Thus, we neglect the productions of those heavier hadrons.

\subsection{Various time scales}

Once energetic hadrons are emitted into the thermal bath, which consists of $\gamma, e^{ \pm}$, and nucleons, hadrons scatter off various background particles and induce cascade processes. Thus, in order to study the propagation of the hadronic particles in the expanding universe, it is important to understand the time scales of various processes; in particular, there are three important processes: (i) hadronic scatterings, (ii) decay (for unstable particles), and (iii) scatterings with thermal plasma through electromagnetic interactions. For the cosmic temperature we consider, the expansion rate is much smaller than the rates for the above processes and hence we can neglect the cosmic expansion in studying the effects of the processes (i) - (iii).

First, we discuss the typical time scale of hadronic scattering processes. The interaction rate for the scattering processes between the projectile hadron $H_{i}$ and the background nucleon $A_{j}$ through $H_{i}+A_{j} \rightarrow A_{k}+\cdots$ is estimated as

$$
\begin{aligned}
\Gamma_{A_{j} \rightarrow A_{k}}^{H_{i}} & =n_{A_{j}} \sigma_{A_{j} \rightarrow A_{k}}^{H_{i}} \beta_{H_{i}} \\
& \simeq\left(4.4 \times 10^{-8} \mathrm{sec}\right)^{-1} f_{A_{j}}\left(\frac{\eta_{10}}{6}\right)\left(\frac{\sigma_{A_{j} \rightarrow A_{k}}^{H_{i}} \beta_{H_{i}}}{40 \mathrm{mb}}\right)\left(\frac{T}{\mathrm{MeV}}\right)^{3},
\end{aligned}
$$

where, in this paper, $H_{i}$ is used for hadrons. Here, $\beta_{H_{i}}$ is the velocity of $H_{i}$, $n_{A_{j}}$ is the number density of the nucleon species $A_{j}, \eta_{10} \equiv \eta \times 10^{10}$, and $f_{A_{j}} \equiv n_{A_{j}} / n_{B}$ with $n_{B}$ being the total baryon number density. For nucleon-nucleon collision processes, experimental data suggest that the product of $\beta$ and $\sigma_{A_{j} \rightarrow A_{k}}^{H_{i}}$ is approximately constant $(\sim 40 \mathrm{mb})$ for large range of the beam energies (see Figs. 11] and 12). Thus, in the following discussion, we sometimes use $40 \mathrm{mb}$ as a typical value of the cross section $\sigma_{A_{j} \rightarrow A_{k}}^{H_{i}}$, although we use energy-dependent experimentally measured cross sections for our numerical calculations. 
The rate for the hadronic scattering process should be compared with the decay rate and also with the stopping rate in the thermal plasma. Among these two, we first consider the decay of the hadrons. Eq. (5.2) shows that the typical time scale for the hadronic scattering processes is longer than $\mathcal{O}\left(10^{-8}\right)$ sec for $T \lesssim 1 \mathrm{MeV}$. Thus, hadrons with lifetime longer than $\sim 10^{-8}$ sec participate in the hadron-hadron collision processes after the BBN starts. Thus, hereafter, we only consider such relatively long-lived mesons $\left(\pi^{ \pm}, K^{ \pm}\right.$, and $\left.K_{L}\right)$ and baryons $(p, \bar{p}, n$, and $\bar{n})$, whose lifetimes are 66.

$$
\begin{aligned}
\tau_{\pi^{ \pm}} & =(2.6033 \pm 0.0005) \times 10^{-8} \mathrm{sec} \\
\tau_{K^{ \pm}} & =(1.2384 \pm 0.0024) \times 10^{-8} \mathrm{sec}, \\
\tau_{K_{L}^{0}} & =(5.17 \pm 0.04) \times 10^{-8} \mathrm{sec} \\
\tau_{n} & =885.7 \pm 0.8 \mathrm{sec} .
\end{aligned}
$$

Other hadrons have very short lifetimes and decay before scattering off the background nuclei. \#

Since the lifetime of the neutron is relatively long, it is necessary to see if the nonthermally produced neutrons may decay before causing the hadrodissociation processes. As will be discussed in the next subsection, at low temperature $T \ll m_{e}$, energy-loss processes via the electromagnetic interaction becomes ineffective for non-relativistic neutrons. Then, such neutron effectively scatters off the background nuclei if the mean-free time is shorter than the lifetime. On the contrary, if the mean-free time is longer than the lifetime, most of the energetic neutrons decay and become protons. Since the proton can be stopped in the thermal bath more easily than the neutrons, the hadrodissociation processes are suppressed in this case.

Once the energy-loss processes via the electromagnetic interactions become ineffective, the effective lifetime of the neutron with energy $E_{n}$ is given by $\gamma_{n} \tau_{n}$ (where $\gamma_{n}=E_{n} / m_{n}$ is the Lorentz factor). Then, the condition for the mean-free time $1 / \Gamma_{A_{j} \rightarrow A_{k}}^{n}$ being shorter than the effective lifetime is given by

$$
T \gg 4.0 \times 10^{-1} \mathrm{keV}\left(\frac{E_{n}}{\mathrm{GeV}}\right)^{-1 / 3}\left(\frac{\sigma_{p \rightarrow N^{\prime}}^{n} \beta_{n}}{40 \mathrm{mb}}\right)^{-1 / 3}\left(\frac{\eta_{10}}{6}\right)^{-1 / 3}
$$

and, for the cosmic time,

$$
t \ll 0.83 \times 10^{7} \sec \left(\frac{E_{n}}{\mathrm{GeV}}\right)^{2 / 3}\left(\frac{\sigma_{p \rightarrow N^{\prime}}^{n} \beta_{n}}{40 \mathrm{mb}}\right)^{2 / 3}\left(\frac{\eta_{10}}{6}\right)^{2 / 3} .
$$

Before closing this subsection, we check that the effects of the cosmic expansion are negligible; this can be seen if the cosmic expansion rate is smaller than the scattering rate

\footnotetext{
${ }^{\# 7}$ For example, lifetimes of $\pi^{0}, K_{S}^{0}$, and $\Lambda^{0}$ are $\tau_{\pi^{0}}=8.4 \times 10^{-17} \mathrm{sec}, \tau_{K_{S}^{0}}=0.89 \times 10^{-10}$, and $\tau_{\Lambda^{0}}=$ $2.63 \times 10^{-10}$ sec, respectively [66].
} 
of the nuclei. Indeed, in the radiation dominated universe with $T \lesssim m_{e}$, the expansion rate is given by

$$
H \simeq(2.6 \mathrm{sec})^{-1} \times\left(\frac{T}{\mathrm{MeV}}\right)^{2}
$$

Thus, the condition $\Gamma_{N \rightarrow N^{\prime}}^{H_{i}} \gg H$ is satisfied when

$$
T \gg 1.7 \times 10^{-2} \mathrm{eV} \times f_{N}^{-1}\left(\frac{\eta_{10}}{6}\right)^{-1}\left(\frac{\sigma_{N \rightarrow N^{\prime}}^{H_{i}}}{40 \mathrm{mb}}\right)^{-1} .
$$

Since we only consider the cases where $X$ decays during the radiation-dominated epoch, this condition is automatically satisfied.

\subsection{Energy-loss of the hadrons via the electromagnetic interac- tions}

As we will discuss in the following sections, energy-loss of the energetic hadrons via the electromagnetic interactions is very important for the calculation of the abundances of the light elements. If energetic hadrons completely lose their energy before scattering off the background nuclei, they do not cause the dissociations of the background nuclei (in particular, $\left.\alpha_{\mathrm{BG}}\right)$. In this case, only exothermic reactions are allowed for the hadronic particles, and the extra-ordinary hadronic inter-converting reactions between protons and neutrons become important. Such processes, which occurs at $t \lesssim 100 \mathrm{sec}$, are discussed in Section [6. On the other hand, if the electromagnetic interactions do not stop the energetic hadrons (in particular, nucleons), they effectively scatter off the background nuclei and induce hadrodissociation. For $t \gtrsim 100 \mathrm{sec}$, this is the case. In particular, (i) $\alpha_{\mathrm{BG}}$ is destructed and various energetic debris nuclei $\left(n, p, \mathrm{D}, \mathrm{T}\right.$, and $\left.{ }^{3} \mathrm{He}\right)$ are produced, (ii) some of these energetic nuclei (in particular, $\mathrm{T},{ }^{3} \mathrm{He}$, and ${ }^{4} \mathrm{He}$ ) scatter off $\alpha_{\mathrm{BG}}$ to produce heavier nuclei $\left({ }^{6} \mathrm{Li},{ }^{7} \mathrm{Li}\right.$, and ${ }^{7} \mathrm{Be}$ ), and (iii) the energetic nucleons emitted during the evolution of hadronic shower inter-convert the background proton and neutron through hadronic collisions. These processes will be discussed in detail in Section 7 and Section 8 ,

In this subsection, we discuss the interaction of the energetic hadronic particles with the background (in particular, photon, electron, and positron) via the electromagnetic interaction. The energetic hadron $H_{i}$ scatters off the background particles via the electromagnetic interaction in particular with the following processes: the Coulomb scattering $\left(H_{i}+e^{ \pm} \rightarrow H_{i}+e^{ \pm}\right)$, the Compton scattering $\left(H_{i}+\gamma \rightarrow H_{i}+\gamma\right)$, the Bethe-Heitler scattering $\left(H_{i}+\gamma \rightarrow H_{i}+e^{+}+e^{-}\right)$, and the photo-pion process $\left(H_{i}+\gamma \rightarrow H_{i}^{\prime}+\pi\right)$. With these processes, energetic hadrons (gradually) lose their energy. The energy-loss rate of $H_{i}$ can be expressed in the following form:

$$
\left(\frac{d E_{H_{i}}}{d t}\right)=\left(\frac{d E_{H_{i}}}{d t}\right)_{\text {Coulomb }}+\left(\frac{d E_{H_{i}}}{d t}\right)_{\mathrm{CP}}+\left(\frac{d E_{H_{i}}}{d t}\right)_{\mathrm{BH}}+\left(\frac{d E_{H_{i}}}{d t}\right)_{\text {photo-pion }},
$$


which is the sum of the various processes listed above. (The concrete expressions of the energy loss rates are given in Appendix B,

For our purpose, it is important to estimate how the hadrons lose their energy in the thermal bath before scattering off the background nuclei. If the energy-loss rate via the electromagnetic processes is large enough, hadrons are stopped before scattering off the background nuclei via the hadronic interactions. To estimate whether a hadron $H_{i}$ is stopped or not through the electromagnetic interaction before scattering off the background proton or $\alpha$, we calculate $\# 8$

$$
R_{A_{j} \rightarrow A_{k}}^{H_{i}}\left(E_{H_{i}}^{(\mathrm{in})}, E_{H_{i}}^{\prime} ; T\right) \equiv n_{A_{j}} \int_{E_{H_{i}}^{(\mathrm{in})}}^{E_{H_{i}}^{\prime}} \sigma_{A_{j} \rightarrow A_{k}}^{H_{i}} \beta_{H_{i}}\left(\frac{d E_{H_{i}}}{d t}\right)^{-1} d E_{H_{i}}
$$

and

$$
R^{H_{i}}\left(E_{H_{i}}^{(\mathrm{in})}, E_{H_{i}}^{\prime} ; T\right) \equiv \sum_{j, k} R_{A_{j} \rightarrow A_{k}}^{H_{i}}\left(E_{H_{i}}^{(\mathrm{in})}, E_{H_{i}}^{\prime} ; T\right)
$$

where $\left(d E_{H_{i}} / d t\right)$ is the energy-loss rate via the electromagnetic interactions which is given in Eq. (5.11), and the sum in Eq. (5.13) is over all the possible hadronic processes. Since $p$ and $\alpha$ are the most abundant in the background among the nuclei, hadronic scattering processes are dominated by the scatterings with $p$ and $\alpha_{\mathrm{BG}}$; in our study, we use the approximation

$$
\sum_{j} n_{A_{j}} \sigma_{A_{j} \rightarrow A_{k}}^{H_{i}}=n_{p} \sigma_{H_{i}+p \rightarrow A_{k}+\cdots}+n_{\alpha} \sigma_{H_{i}+\alpha \rightarrow A_{k}+\cdots}
$$

$R^{H_{i}}\left(E_{H_{i}}^{(\mathrm{in})}, E_{H_{i}}^{\prime} ; T\right)$ parameterizes the number of the hadronic scatterings before the energy of the hadron $H_{i}$ with its initial energy $E_{H_{i}}^{(\text {in) }}$ decreases to $E_{H_{i}}^{\prime}$ via the electromagnetic interactions. Thus, if $R^{H_{i}}\left(E_{H_{i}}^{(\mathrm{in})}, E_{H_{i}}^{(\mathrm{th})} ; T\right) \lesssim 1$ (with $E_{H_{i}}^{(\text {th) }}$ being the threshold energy of some hadrodissociation process), the high energy hadron $H_{i}$ is (mostly) stopped and does not cause the hadrodissociation process. On the contrary, if $R^{H_{i}}\left(E_{H_{i}}^{(\mathrm{in})}, E_{H_{i}}^{(\mathrm{th})} ; T\right)$ is larger than unity, the high energy hadrons are not stopped through the electromagnetic interaction and cause hadrodissociation processes. In particular, if $R^{H_{i}}\left(E_{H_{i}}^{(\mathrm{in})}, E_{H_{i} \alpha}^{(\mathrm{th})} ; T\right) \gtrsim 1$ with $E_{H_{i} \alpha}^{(\text {th) }}$ being the threshold energy for the destruction process of $\alpha_{\mathrm{BG}}$, high energy projectile hadron with its initial energy $E_{H_{i}}^{(\mathrm{in})}$ effectively destroys $\alpha_{\mathrm{BG}}$. The number density of the $\alpha_{\mathrm{BG}}$ becomes abundant after the cosmic time $t \sim 200 \mathrm{sec}(T \sim 0.1 \mathrm{MeV})$. Thus, if $X$ decays after this epoch, abundances of the light elements (in particular, D and ${ }^{3} \mathrm{He}$ ) may be significantly changed by the direct destruction of $\alpha_{\mathrm{BG}}$.

Using the quantity $R^{H_{i}}$ given in Eq. (15.13) , we estimate the energy of $H_{i}$ just before scattering off the background proton or $\alpha_{\mathrm{BG}}$. For stable particles, such a energy for a

\footnotetext{
${ }^{\# 8} R^{H_{i}}$ depends also on the ${ }^{4}$ He fraction $Y$. We properly take into account the $Y$ dependence in our numerical calculations.
} 


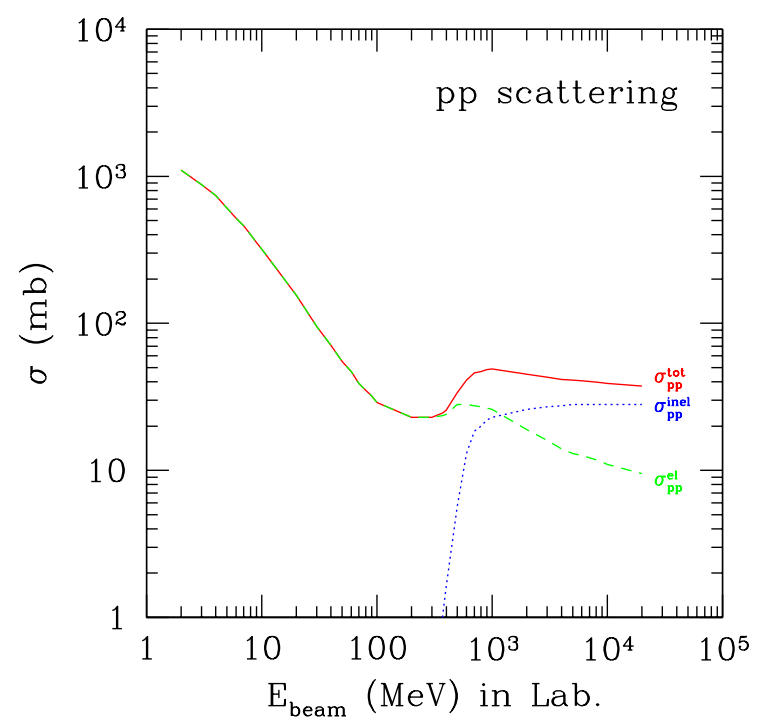

Figure 11: Cross sections of the $p p$ scattering processes as functions of the kinetic energy of the beam (projectile) proton in the laboratory system. The solid line is the total cross section while the dashed (dotted) line is the elastic (inelastic) cross section.

given initial energy $E_{H_{i}}^{(\mathrm{in})}$, which is denoted as $\tilde{E}_{H_{i}}^{(R=1)}\left(E_{H_{i}}^{(\mathrm{in})}\right)$, is estimated by solving the following relation

$$
R^{H_{i}}\left(E_{H_{i}}^{(\mathrm{in})}, \tilde{E}_{H_{i}}^{(R=1)} ; T\right)=1 .
$$

For the neutron, we should take account of the fact that the neutron may decay before scattering off the background nuclei. Thus, for the neutron, we define $\tilde{E}_{n}^{(R=1)}\left(E_{n}^{(\mathrm{in})}\right)$ as follows. For the given initial energy of the neutron $E_{n}^{(\mathrm{in})}$, we calculate the Lorentz factor $\gamma_{n}^{(\mathrm{in})} \equiv E_{n}^{(\mathrm{in})} / m_{n}$ as well as the total scattering rate for the hadronic processes $\Gamma_{\text {had }}^{n} \equiv$ $n_{p} \sigma_{n+p \rightarrow \cdots}+n_{\alpha} \sigma_{n+\alpha \rightarrow \cdots}$. (Here, the cross sections are estimated with the initial energy of the neutron.) Then, if $\Gamma_{\text {had }}^{n}>\left(\gamma_{n}^{(\text {in })} \tau_{n}\right)^{-1}$, we expect that the neutron scatters off the background nuclei before it decays, and we estimate $\tilde{E}_{n}^{(R=1)}\left(E_{n}^{(\mathrm{in})}\right)$ using Eq. (5.15). On the contrary, if $\Gamma_{\text {had }}^{n}<\left(\gamma_{n}^{(\text {in) }} \tau_{n}\right)^{-1}$, neutron is likely to decay before scattering off the background nuclei. In this case, the energetic neutron is equivalent to the proton for the calculation of the hadrodissociation processes. Thus, for this case we use the relation $\tilde{E}_{n}^{(R=1)}\left(E_{n}^{(\text {in })}\right)=\tilde{E}_{p}^{(R=1)}\left(E_{n}^{(\text {in })}\right) . \# 9$

For various nuclei, we calculate $\tilde{E}_{H_{i}}^{(R=1)}$ as functions of the initial energy $E_{H_{i}}^{(\mathrm{in})}$. In order to precisely calculate $\tilde{E}_{H_{i}}^{(R=1)}$, we need informations about the cross sections for the

${ }^{\# 9}$ Neutron loses its energy before it decays and hence the energy of the proton produced by the neutron is not exactly equal to $E_{n}^{(i n)}$. However, the energy-loss rate of the neutron via the electromagnetic processes is much less efficient than that of the proton and hence we can neglect the energy-loss of the neutron before it decays. 


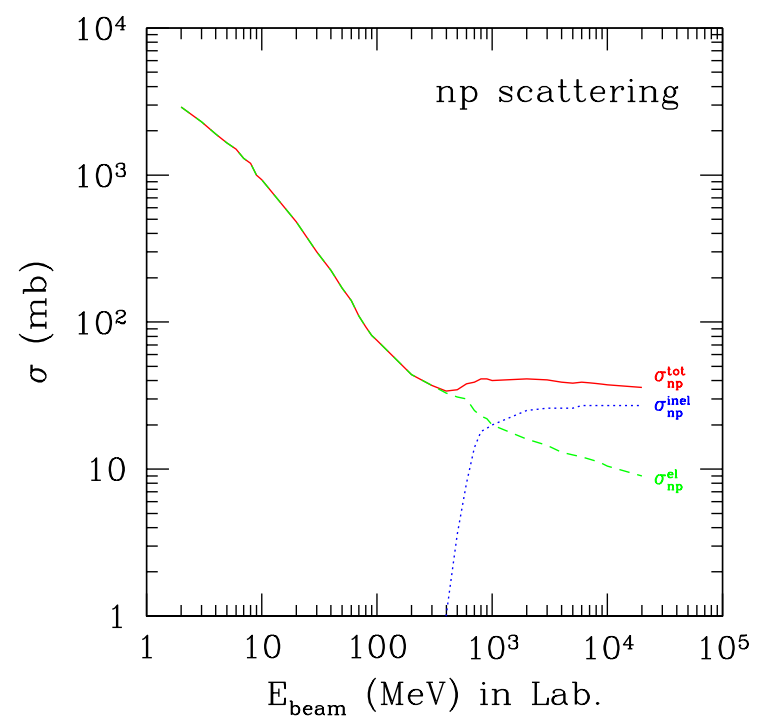

Figure 12: Cross sections of the $n p$ scattering processes as functions of the kinetic energy of the beam (projectile) proton in the laboratory system. The solid line is the total cross section while the dashed (dotted) line is the elastic (inelastic) cross section.

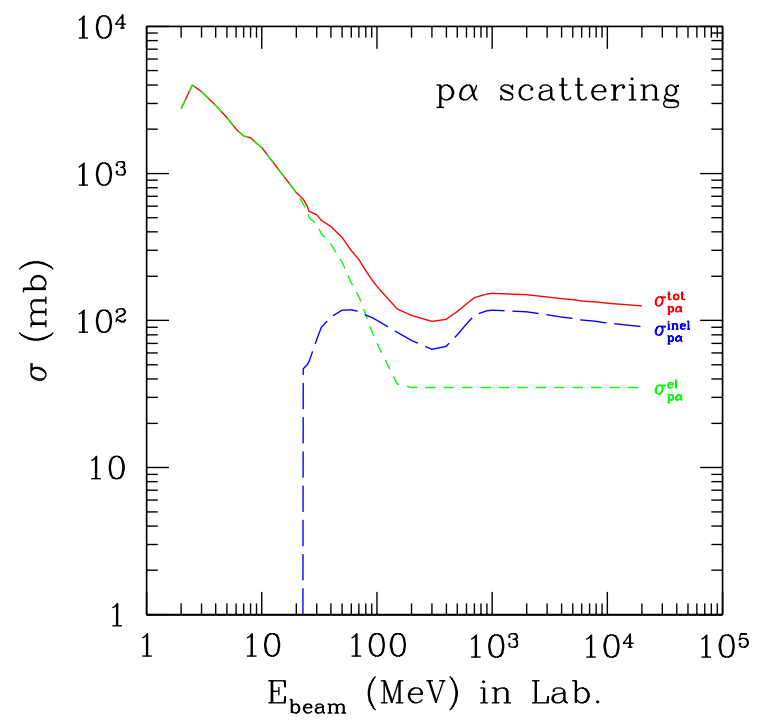

Figure 13: Cross sections of the $p \alpha$ scattering processes as functions of the kinetic energy of the beam (projectile) proton in the laboratory system. The solid line is the total cross section while the dashed (dotted) line is the elastic (inelastic) cross section. 


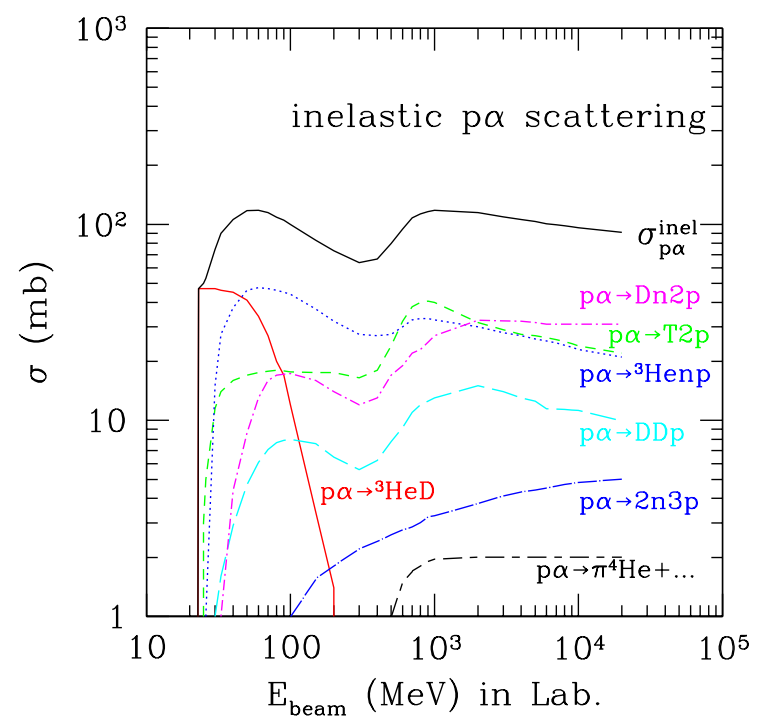

Figure 14: Cross sections of the inelastic $p \alpha$ scattering processes.

hadronic processes. For energetic proton and neutron, we use detailed experimental data of cross sections for $p p, n p$, and $p \alpha$ collisions [69]. In Figs. 11, 12, 13, and 14, we plot the data of total, elastic and inelastic cross sections for these collision processes, respectively.

In Fig. 15, we plot the contours of the constant $\tilde{E}_{p}^{(R=1)}$ on the $T$ vs. $E_{p}^{(i n)}$ plane. Here we use $\eta=6.1 \times 10^{-10}$ and $Y=0.25$. From this figure, we see that the protons are completely stopped when the temperature is sufficiently high. This is because the number of the background electron is not Boltzmann-suppressed at high temperature, resulting in enhanced energy-loss rate.

For a more quantitative discussion, it is convenient to define the typical energy-loss rate through the electromagnetic processes:

$$
\Gamma_{\mathrm{EM}}^{H_{i}} \equiv \frac{1}{E_{\mathrm{kin}}} \frac{d E_{H_{i}}}{d t} .
$$

If $\Gamma_{\mathrm{EM}}^{H_{i}}$ is larger than the hadronic scattering rate, energy loss is effective and the energy of the hadron is significantly reduced before scattering off the background nuclei. On the contrary, if $\Gamma_{\mathrm{EM}}^{H_{i}} \lesssim \Gamma_{A_{j} \rightarrow A_{k}}^{H_{i}}$, energy loss is not important.

At the temperature $T \sim \mathcal{O}(10-100) \mathrm{keV}$, Coulomb scattering is the most important for the energy loss. Using the energy-loss rates given in Appendix $\mathbb{B}$, we can estimate $\Gamma_{\mathrm{EM}}^{p}$ for the case where the Coulomb scattering is the dominant process:

$$
\left[\Gamma_{\mathrm{EM}}^{p}\right]_{\mathrm{Coulomb}} \simeq\left(1.4 \times 10^{-14} \mathrm{sec}\right)^{-1} \times \beta_{p}^{-1} \Lambda\left(\frac{E_{\mathrm{kin}}}{m_{N}}\right)^{-1} x_{e}^{-3 / 2} e^{-x_{e}},
$$

where $x_{e} \equiv m_{e} / T$. (Here, we consider protons with $\beta_{p}$ larger than the thermal velocity of the background electron, in which we are mostly interested at this temperature.) Since 


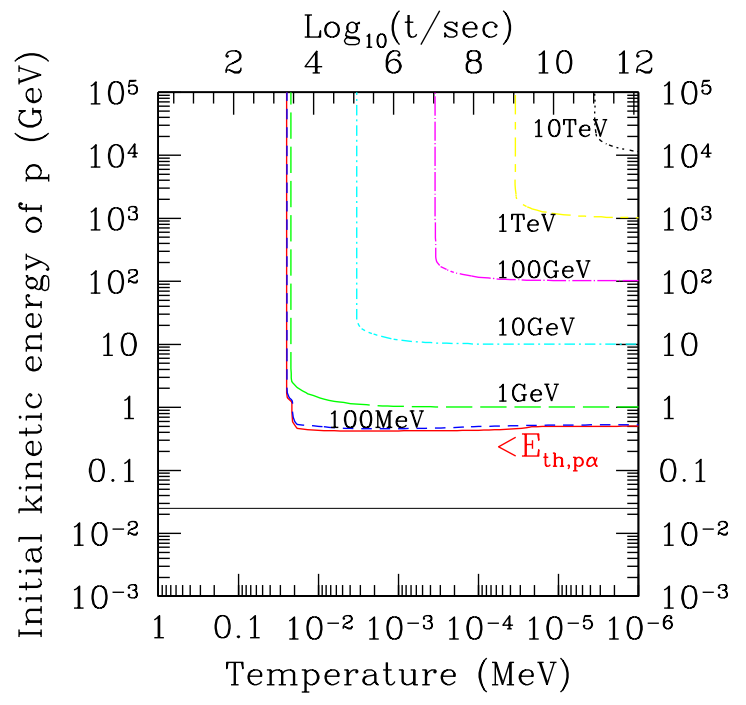

Figure 15: Contours of the constant $\tilde{E}_{p}^{(R=1)}$, the energy of the proton just before it scatters off the background nuclei ( $p$ or ${ }^{4} \mathrm{He}$ ). The horizontal axis is the temperature while the vertical one is the initial energy of the proton $E_{p}^{(\mathrm{in})}$. The numbers in the figure are the values of $\tilde{E}_{p}^{(R=1)}$, and the solid line with " $<E_{\mathrm{th}, p \alpha}$ " shows the contour for $\tilde{E}_{p}^{(R=1)}$ being equal to the threshold energy for the destruction process of ${ }^{4} \mathrm{He}, E_{p \alpha}^{(\mathrm{th})}=25 \mathrm{MeV}$. The horizontal thin-solid line denotes $E_{p}^{(\mathrm{in})}=E_{p \alpha}^{(\mathrm{th})}$. Here we use $\eta=6.1 \times 10^{-10}$ and $Y=0.25$.

the number density of the electron becomes smaller as the temperature gets decreased, $\Gamma_{\mathrm{EM}}^{p}$ in this case decreases as the temperature becomes lower. Comparing Eq. (5.17) with Eq. (5.2), we can see that the proton is completely stopped when $T \gtrsim \mathcal{O}(10) \mathrm{keV}$.

For ultra-relativistic protons, in fact, the Compton energy-loss (and the Bethe-Heitler process) becomes effective in particular at lower temperature. Typical behavior given in Fig. [15] is indeed understood by using the formula for, e.g., the Compton process. Using Eq. (B.10), we can obtain $\Gamma_{\mathrm{EM}}^{p}$ for the ultra-relativistic case:

$$
\left[\Gamma_{\mathrm{EM}}^{p}\right]_{\text {Compton }} \simeq(13 \mathrm{sec})^{-1} \times\left(\frac{E_{\mathrm{kin}}}{100 \mathrm{GeV}}\right)\left(\frac{T}{1 \mathrm{keV}}\right)^{4}
$$

Thus, for the ultra-relativistic protons, we can see that the energy loss is effective for the temperature $T \gtrsim \mathcal{O}(0.1) \mathrm{keV} \times\left(E_{\text {kin }} / 100 \mathrm{GeV}\right)^{-1}$.

For the neutron, we plot the contours of constant $\tilde{E}_{n}^{(R=1)}$ in Fig. 16. For the $n \alpha$ scatterings, we use the cross section for $p \alpha$ processes assuming the isospin symmetry as well as small number of data for $n \alpha$ scatterings. Empirically, the size of the isospin breaking in this case is estimated to be within $20 \%(10 \%)$ for $E_{n}^{(\text {in })}=25 \mathrm{MeV}(100 \mathrm{MeV})$. As we will discuss later, we will adopt $20 \%$ errors to all the hadronic cross sections in 


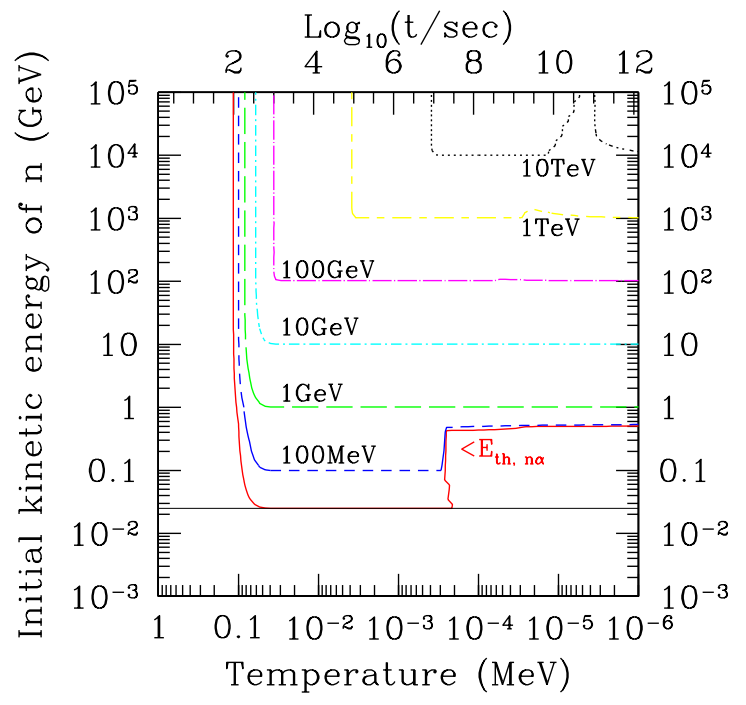

Figure 16: Same as Fig. 15, but for neutron. (The horizontal thin-solid line denotes $E_{n}^{(\mathrm{in})}=E_{n \alpha}^{(\mathrm{th})}$ accordingly.)

our Monte Carlo analysis, which will also take account of this uncertainty related to the isospin breaking.

As one can see, non-relativistic neutrons are effectively stopped when the temperature is so high that the number density of the background electron is large enough. For the complete stopping of the neutron, the temperature should be higher than $\sim 100 \mathrm{keV}$, which is slightly higher than the stopping temperature of the proton; using Eq. (B.19), one can calculate $\Gamma_{\mathrm{EM}}^{n}$ and see that $\Gamma_{\mathrm{EM}}^{n}$ for non-relativistic neutron becomes smaller than the hadronic scattering rate when $T \lesssim 100 \mathrm{keV}$. Thus, at lower temperature, energy-loss of the neutron becomes inefficient. When the temperature becomes low enough, however, time scale for the hadronic scattering becomes longer than the lifetime of the neutron. This is the reason of the drastic change of $\tilde{E}_{n}^{(R=1)}$ at the temperature $T \sim 0.3 \mathrm{keV}$ for non-relativistic neutron.

We also calculate the typical energy of D, T, ${ }^{3} \mathrm{He}$ and ${ }^{4} \mathrm{He}$ just before scattering off the background nuclei. We plot the contours of $\tilde{E}_{\mathrm{D}}^{(R=1)}, \tilde{E}_{\mathrm{T}}^{(R=1)}, \tilde{E}_{3}^{(R=1)}$ and $\tilde{E}_{4}^{(R=1)}$ in Figs. 17 , [18, 19] and 20, respectively. (For readers' convenience, we also plot the cross sections for the $p \mathrm{D}$ and $p^{3} \mathrm{He}$ processes in Figs. 21 and 22, respectively.) For these charged particles $A_{i}$, behavior of $\tilde{E}_{A_{i}}^{(R=1)}$ is similar to the case of the proton.

In this paper, we approximate that all the hadrons $H_{i}$ have the energy $\tilde{E}_{H_{i}}^{(R=1)}$ just before they scatter off the background proton or $\alpha_{\mathrm{BG}}$. 


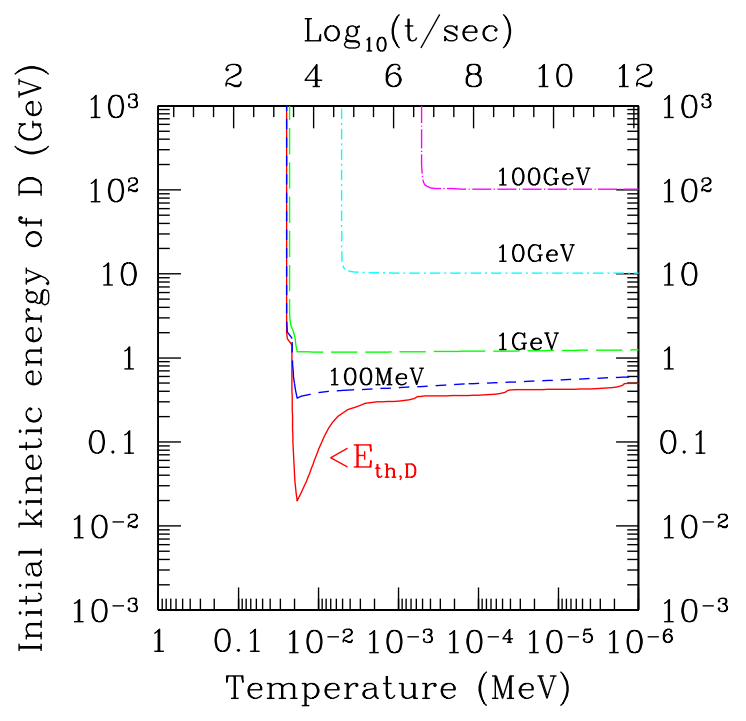

Figure 17: Same as Fig. 15, but for D. The solid line with " $<E_{\mathrm{th}, \mathrm{D}}$ " shows the contour for $\tilde{E}_{\mathrm{D}}^{(R=1)}$ being equal to the threshold energy for the destruction process $\mathrm{D}+p_{\mathrm{BG}} \rightarrow 2 p+n$.

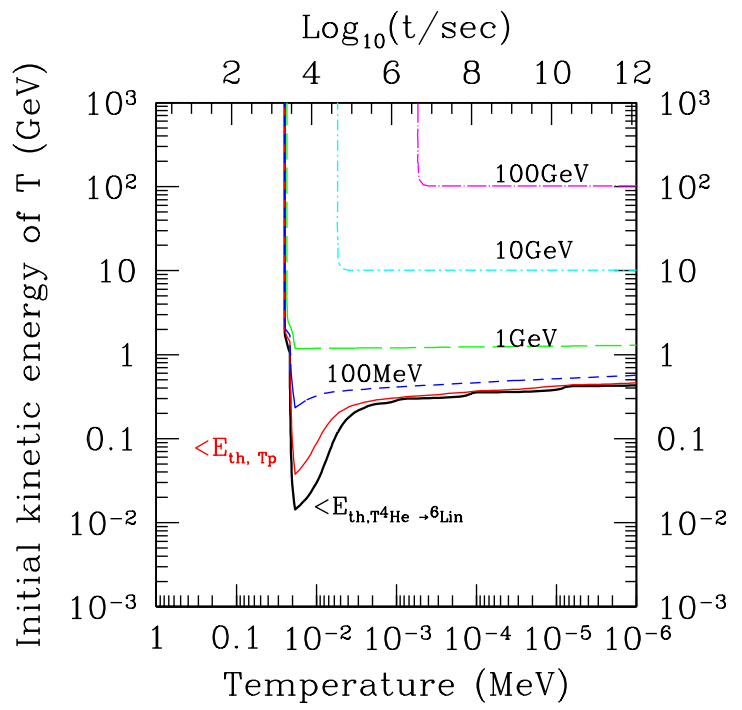

Figure 18: Same as Fig. 15, but for T. The solid line with " $<E_{\mathrm{th}, \mathrm{T} p}$ " shows the contour for $\tilde{E}_{\mathrm{T}}^{(R=1)}$ being equal to the threshold energy for the destruction process of the projectile $\mathrm{T}$ through $\mathrm{T}+p_{\mathrm{BG}}$, while the thick solid line is the contour for $\tilde{E}_{\mathrm{T}}^{(R=1)}$ being equal to the threshold energy for the process $\mathrm{T}+\alpha_{\mathrm{BG}} \rightarrow{ }^{6} \mathrm{Li}+n$. 


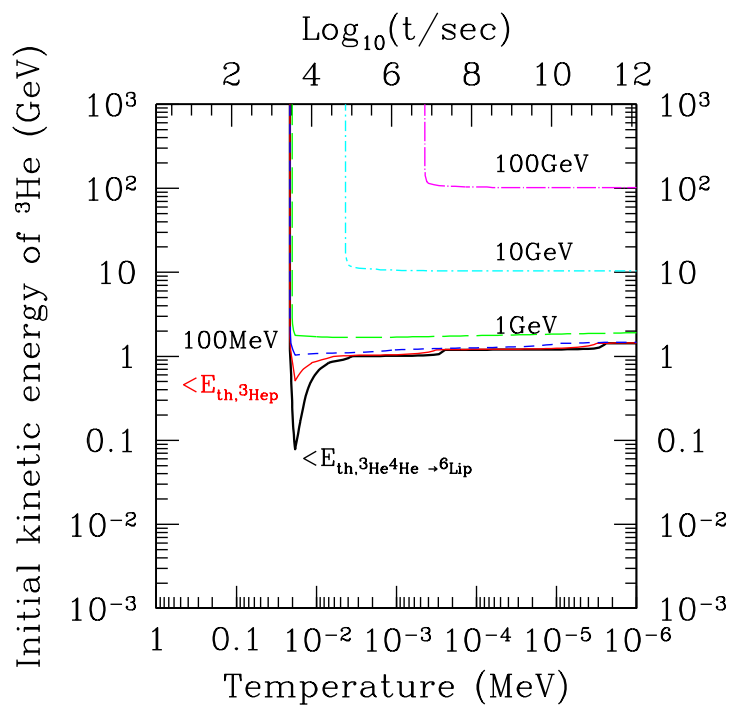

Figure 19: Same as Fig. 15, but for ${ }^{3} \mathrm{He}$. The solid line with " $<E_{\mathrm{th},{ }^{3} \mathrm{He} p}$ " shows the contour for $\tilde{E}_{3 \mathrm{He}}^{(R=1)}$ being equal to the threshold energy for the destruction process of the projectile ${ }^{3} \mathrm{He}$ through ${ }^{3} \mathrm{He}+p_{\mathrm{BG}}$, while the thick solid line is the contour for $\tilde{E}_{3}^{(R=1)}$ being equal to the threshold energy for the process ${ }^{3} \mathrm{He}+\alpha_{\mathrm{BG}} \rightarrow{ }^{6} \mathrm{Li}+p$.

\section{Inter-Conversion between Neutron and Proton}

So far, we have discussed the propagation of the hadronic particles in the expanding universe, paying particular attention to their energy-loss. Those particles cause various hadronic processes before and after being stopped by the electromagnetic scatterings and affect the abundances of the light elements.

The first effect we discuss is the inter-converting $p \leftrightarrow n$ reaction caused by hadrons emitted from decaying $X$. \#10 Implications of such effect was first considered in Ref. [7. In our study, we basically follow the strategy of Ref. [7] with several improvements, which are discussed in this section.

Especially, for relatively early epoch $T \gtrsim 0.1 \mathrm{MeV}$ (i.e., $t \lesssim 100 \mathrm{sec}$ ), the emitted hadrons extraordinarily inter-convert the ambient protons and neutrons by the strong interaction even after the conventional freeze-out time of the neutron in the SBBN. Thus, for massive particle $X$ with relatively short lifetime $\left(\tau_{X} \sim\left(10^{-2}-100\right)\right.$ sec $)$, the interconversion effect may induce a significant change of the light-element abundances. In particular, since the proton is more abundant than the neutron, $n / p$ ratio is enhanced if such inter-conversion is effective, resulting in overproduction of ${ }^{4} \mathrm{He}$. \#11 Thus, in this section,

${ }^{\# 10}$ Of course, pions generated from the high-energy photons via the process $\gamma+\gamma_{\mathrm{BG}} \rightarrow \pi^{+}+\pi^{-}$are also included in the present work.

${ }^{\# 11} \mathrm{D} / \mathrm{H}$ and ${ }^{7} \mathrm{Li} / \mathrm{H}$ are also sensitive to $n / p$ even at $t \gtrsim 100 \mathrm{sec}$. This effect is studied together with 


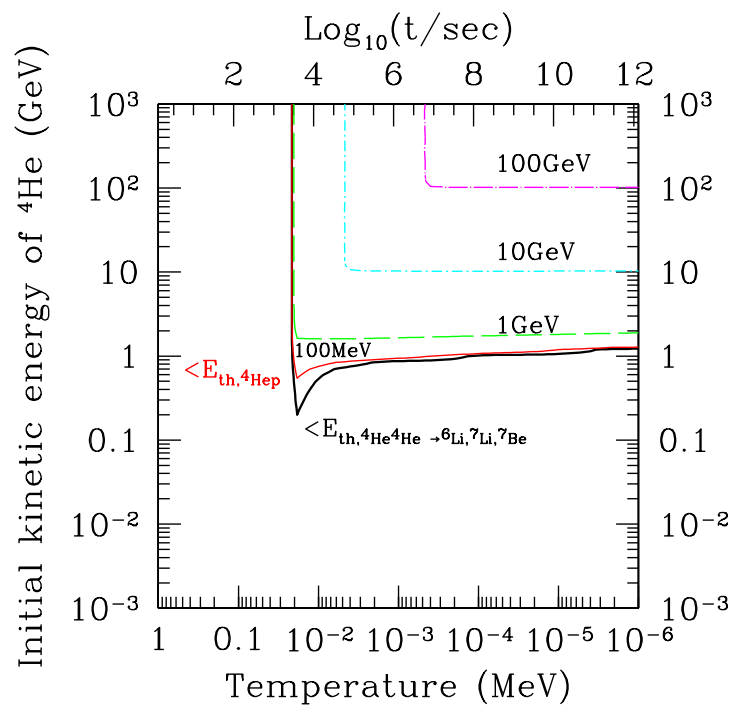

Figure 20: Same as Fig. [15] but for ${ }^{4} \mathrm{He}$. The solid line with " $<E_{\mathrm{th},{ }^{4} \mathrm{Hep}}$ " shows the contour for $\tilde{E}_{4_{\mathrm{He}}^{(R)}}^{(R)}$ being equal to the threshold energy for the destruction process of the projectile ${ }^{4} \mathrm{He}$ through ${ }^{4} \mathrm{He}+p_{\mathrm{BG}}$, while the thick solid line denotes the contour for $\tilde{E}_{4 \mathrm{He}}^{(R=1)}$ being equal to the threshold energy for the process ${ }^{4} \mathrm{He}+\alpha_{\mathrm{BG}} \rightarrow{ }^{7} \mathrm{Li}+\cdots$.

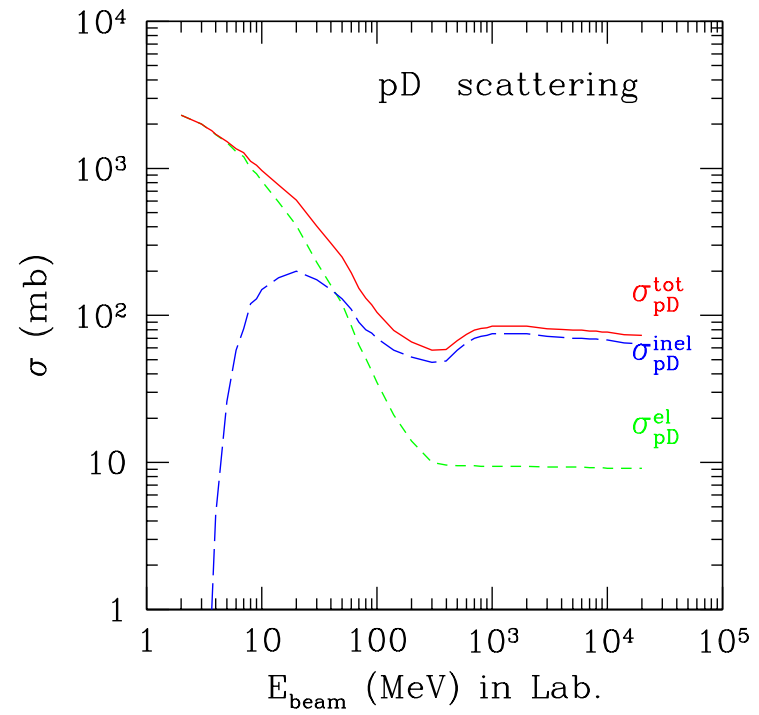

Figure 21: Cross sections of the $p \mathrm{D}$ scattering processes. 


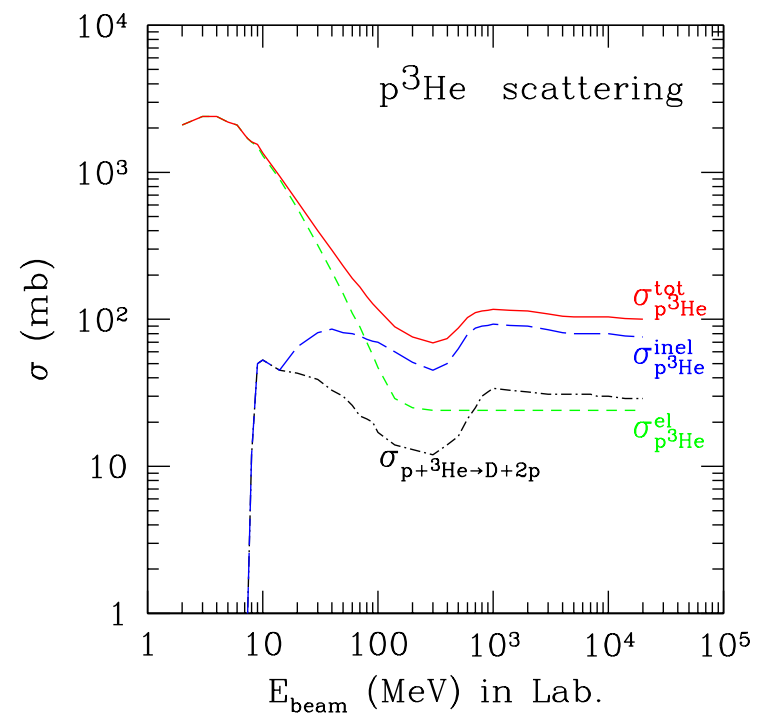

Figure 22: Cross sections of the $p^{3} \mathrm{He}$ scattering processes.

we discuss the inter-conversion processes. In particular, we present the thermally averaged cross sections for the relevant processes. In our analysis, for all the inter-conversion cross sections given in this section, we adopt $50 \%$ uncertainties when we perform the Monte Carlo analysis.

\subsection{Cross sections of hadron-nucleon scattering}

First, let us summarize the cross sections for the relevant reactions. As we have discussed in the previous sections, once a high energy hadron is injected into the thermal bath at the early stage of the BBN (more precisely, when $T \gtrsim 0.1 \mathrm{MeV}$ ), energy-loss processes via the electromagnetic interactions are very effective (except for the neutral kaon). Thus, even if the non-thermally produced hadrons (in particular, $\pi^{ \pm}, K^{ \pm}, p$, and $n$ ) are quite energetic when they are produced, they are quickly thermalized. In this case, the typical kinetic energy of these hadrons becomes $\sim T$. Thus, for the inter-conversion processes, only the exothermic reactions are relevant since the kinetic energy of the thermalized hadrons are expected to be too small to induce endothermic reactions. In addition, the inter-conversion processes occur mostly with very small velocity.

Concerning the exothermic reactions, it is well-known that the cross section $\sigma$ is approximately inversely proportional to the velocity $\beta$ when the velocity is very small; namely $\sigma \beta$ becomes almost constant. Thus, we use the threshold cross section. The cross sections given in the following are from Ref. [7].

hadrodissociation effect in the next section. 
The thermally-averaged cross sections for the processes $\pi^{+}+n \rightarrow p+\pi^{0}$ and $\pi^{-}+p \rightarrow$ $n+\pi^{0}$ are given by

$$
\begin{aligned}
& \langle\sigma \beta\rangle_{n \rightarrow p}^{\pi^{+}}=1.7 \mathrm{mb}, \\
& \langle\sigma \beta\rangle_{p \rightarrow n}^{\pi^{-}}=1.5 C_{\pi}^{2}(T) \mathrm{mb},
\end{aligned}
$$

respectively. Here, $C_{H_{i}}^{2}(T)$ is the Coulomb correction factor which takes account of the modification of the wave-function of the charged particle due to the Coulomb field. For the opposite-sign charged particles, Coulomb correction factor is given by [70]

$$
C_{H_{i}}^{2}(T)=\frac{2 \pi \alpha_{\mathrm{em}} \sqrt{\mu_{H_{i}} / 2 T}}{1-e^{-2 \pi \alpha_{\mathrm{em}} \sqrt{\mu_{H_{i}} / 2 T}}},
$$

where $\alpha_{\mathrm{em}}$ is the fine structure constant and $\mu_{H_{i}}$ is the reduced mass of the hadron $H_{i}$ and the target nucleon.

Next, we consider the effects of the nucleons and anti-nucleons directly produced by the decay of $X$. In our study, we only consider the case where the numbers of such $\bar{p}$ and $\bar{n}$ produced by the hadronic decay of $X$ are individually the same as those of $p$ and $n$.

When the temperature is high enough, nucleons are stopped in the thermal bath by the electromagnetic interactions. (Such temperature is given by $T \gtrsim 20 \mathrm{keV}$ and $100 \mathrm{keV}$ for (anti-) proton and (anti-) neutron, respectively.) In such a case, we treat nucleonanti-nucleon pair $N_{i} \bar{N}_{i}$ like a meson, following Ref. [7]. Then the $N_{i} \bar{N}_{i}$ meson induces the inter-conversion processes: $N_{i} \bar{N}_{i}+N_{j} \rightarrow N_{j}^{\prime}+\cdots$. If the nucleons are thermalized, we can use the threshold cross sections:

$$
\begin{aligned}
\langle\sigma \beta\rangle_{n \rightarrow n}^{n \bar{n}} & =37 \mathrm{mb}, \\
\langle\sigma \beta\rangle_{p \rightarrow n}^{n \bar{n}} & =28 \mathrm{mb}, \\
\langle\sigma \beta\rangle_{n \rightarrow p}^{p \bar{n}} & =28 \mathrm{mb}, \\
\langle\sigma \beta\rangle_{p \rightarrow p}^{p \bar{p}} & =37 C_{p}^{2}(T) \mathrm{mb} .
\end{aligned}
$$

When the temperature becomes lower, on the contrary, nucleons cannot be easily stopped. Then, they also induce the hadrodissociation processes and hence effects of the anti-nucleons cannot be studied just by taking account of the conversion effects. Importantly, however, once the hadrodissociation processes become effective, a large number of protons and neutrons are produced while the anti-nucleons are not produced in the hadronic shower. In this case, anti-nucleons directly produced by the decay of $X$ become irrelevant since their numbers are much smaller than those of non-thermally produced nucleons. Thus, when most of the energetic nucleons scatter off the background nuclei before being stopped, inter-conversion by the anti-nucleons becomes unimportant. When the anti-nucleons are not stopped, it is difficult to estimate their energies with which the inter-conversion cross sections should be evaluated. Thus, we do not include the interconversion process due to the anti-nucleon in such a case. Notice that we have numerically 
checked that the resultant constraints on the properties of $X$ does not change even if we include the inter-conversion by the anti-nucleons with the threshold cross sections at such a later epoch.

Finally, we comment on our treatment of the kaons. Since the kaons have relatively long lifetimes, they may also contribute to the inter-conversion processes. Unfortunately, however, cross sections of many of the conversion processes related to the kaons are not available. In addition, the neutral kaon $K_{L}^{0}$ is hardly stopped in the thermal bath and hence it is difficult to estimate its effects on the inter-conversion without making some assumptions. In fact, in Refs. [7, 15], effects of the inter-conversion by the kaons are studied with some assumptions and approximations. We have followed the method given in those references and estimated the effects of the kaons. Then, we have found that, with the procedure given in Refs. [7, 15], our resultant constraint (i.e., upper bound on the parameter $\left.m_{X} Y_{X}\right)$ from the over production of ${ }^{4} \mathrm{He}$ becomes slightly severer. In our analysis, however, we neglect the inter-conversion effects of the kaons since the inclusion of the kaons introduce some uncontrollable uncertainties. Effects of the kaons are expected to make the constraints severer and hence, in order to derive conservative constraints, our treatment of the kaons is justified.

\subsection{Formulation}

In this subsection, we formulate the time evolution equations with the late-time "meson" injection. As we have mentioned, the hadron injection at the beginning of the BBN epoch enhances the inter-converting reactions between neutron and proton, and the freeze-out value of $n / p$ may be increased. The inter-conversion term in the Boltzmann equations for the number density of the nucleon $N(=p, n)$ is written as

$$
\left[\frac{d n_{N}}{d t}\right]_{\mathrm{IC}}=-B_{h} \Gamma_{X} n_{X} \sum_{N^{\prime}}\left(K_{N \rightarrow N^{\prime}}-K_{N^{\prime} \rightarrow N}\right),
$$

where $K_{N \rightarrow N^{\prime}}$ denotes the averaged number of the transition $N \rightarrow N^{\prime}$ per one hadronic decay of $X$.

The averaged number of the transition $N \rightarrow N^{\prime}$ for one hadronic decay of $X$ is expressed as

$$
K_{N \rightarrow N^{\prime}}=\sum_{H_{i}} N^{H_{i}} P_{N \rightarrow N^{\prime}}^{H_{i}}
$$

where $H_{i}$ runs over the hadronic species which are relevant to the nucleon inter-converting reactions (i.e., $H_{i}=\pi^{+}, \pi^{-}, n \bar{n}$ and $p \bar{p}$ ). In addition, $N^{H_{i}}$ is the averaged number of $H_{i}$ produced by one hadronic decay of $X$. Note that we assume that two hadron jets are produced in one decay of $X$ in this section. $N^{H_{i}}$ is shown in Fig. 9] as a function of $2 E_{\text {jet }}$, where $E_{\text {jet }}$ is the energy of one hadron jet. ${ }^{\# 12}$ Furthermore, $P_{N \rightarrow N^{\prime}}^{H_{i}}$ denotes the probability

\footnotetext{
\#12 Here, we only consider the effects of the "mesons" $\left(\pi^{+}, \pi^{-}, n \bar{n}\right.$ and $\left.p \bar{p}\right)$ directly produced by the
} decay of $X$. Notice that, for the period when the inter-conversion becomes important, the background 


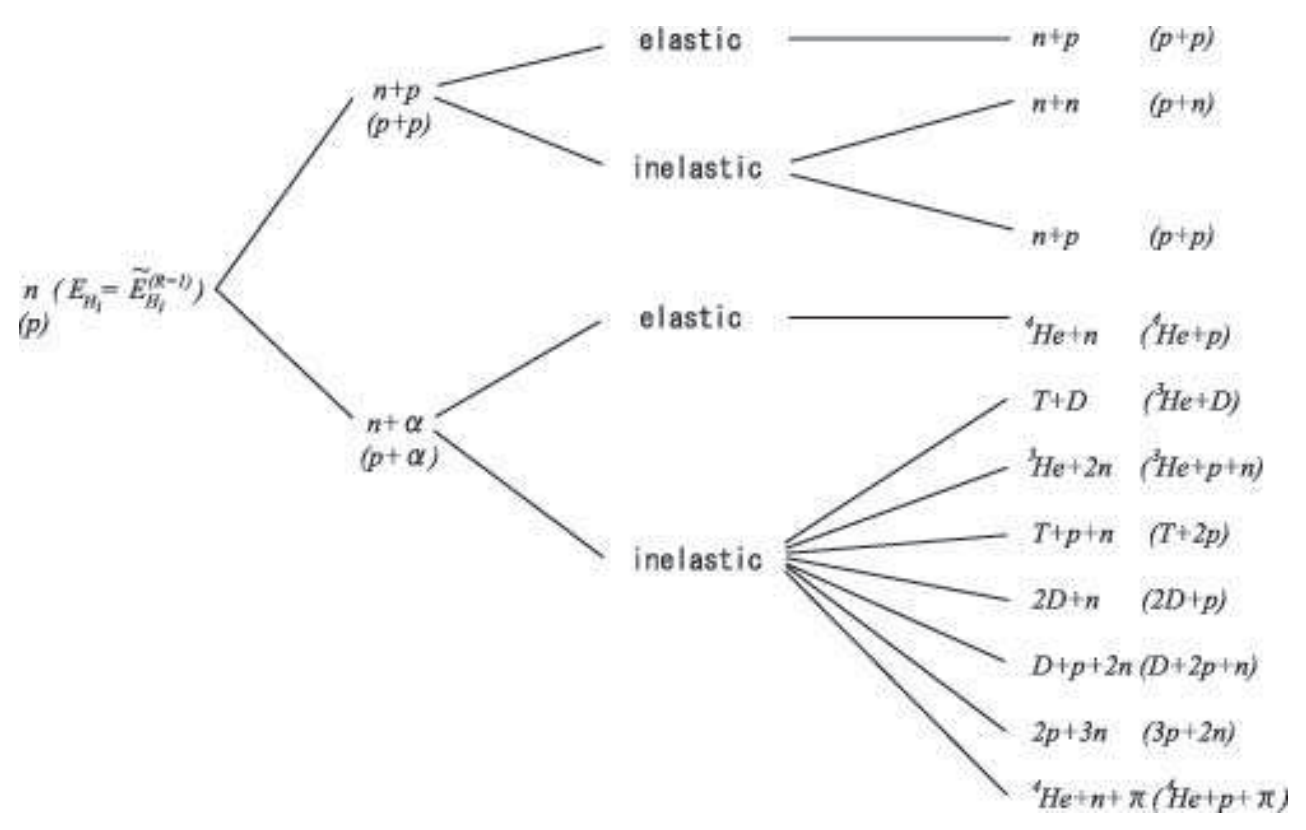

Figure 23: Schematic picture of hadron shower induced by a high energy neutron (proton) which scatters off the background proton or the background $\alpha_{\mathrm{BG}}$.

that a hadron species $H_{i}$ induces the nucleon transition $N \rightarrow N^{\prime}$ and is represented by

$$
P_{N \rightarrow N^{\prime}}^{H_{i}}=\frac{\Gamma_{N \rightarrow N^{\prime}}^{H_{i}}}{\Gamma_{\mathrm{dec}}^{H_{i}}+\Gamma_{\mathrm{abs}}^{H_{i}}},
$$

where $\Gamma_{\text {dec }}^{H_{i}}$ is the decay rate of $H_{i}$, and $\Gamma_{\text {abs }}^{H_{i}} \equiv \Gamma_{p \rightarrow p}^{H_{i}}+\Gamma_{p \rightarrow n}^{H_{i}}+\Gamma_{n \rightarrow p}^{H_{i}}+\Gamma_{n \rightarrow n}^{H_{i}}$ is the total absorption rate.

\section{$7 \quad$ Hadrodissociation of Background $\alpha$}

\subsection{Basic equations}

As was discussed in Section [5, when $R^{H_{i}}$ is more than unity, the hadronic scattering processes between the emitted high-energy nucleons and the background proton or ${ }^{4} \mathrm{He}$ (called $\alpha_{\mathrm{BG}}$ ) become effective. In particular, $\alpha_{\mathrm{BG}}$ can be destroyed and energetic nuclei, like $\mathrm{D}, \mathrm{T},{ }^{3} \mathrm{He}$ are produced. We call this type of the hadronic destruction "hadrodissociation."

temperature is so high that the hadrons we are interested in are stopped before scattering off the background nuclei. Thus, the hadronic shower does not occur in this case, and we do not have to consider the secondary production of the mesons and baryons. 
In order to study the effects of the hadrodissociation, we follow the approach of Ref. [8] with several improvements. In Fig. 23, we show the schematic picture of the hadronic shower induced by a high-energy neutron and proton. Hereafter, we discuss how we study the processes shown in Fig. 23.

Before going to the main discussion, however, let us comment on the treatment of our high-energy anti-nucleons. In our analysis, we neglect hadrodissociation processes induced by high-energy anti-protons and anti-neutrons since we do not have sufficient experimental data for the scattering processes between an energetic anti-nucleon and a nucleus. Such anti-nucleons may change the abundances of the light elements by dissociating background nuclei (as well as by the inter-conversion effect which was discussed in Section [6). We expect, however, that the resultant constraints do not change much by this approximation since the anti-nucleons are not produced secondarily in the hadronic shower. Of course, such energetic anti-nucleon directly produced by the decay of $X$ may produce energetic hadrons by scattering off the background nuclei, which may evolve into a hadronic shower. Number of such a process is at most the same as that of the hadronic shower induced by the energetic $p$ and $n$ and hence the numbers of the hadrodissociation processes may be underestimated at most by a factor of two. Since, as we often mentioned, we do not have sufficient experimental data to study such processes, we neglect the energetic anti-nucleons to derive conservative constraints.

Although the hadronic shower contains multiple scatterings of the hadrons in the thermal bath, the evolution of the shower can be followed by taking account of two types of elementally processes, which are both discussed in the previous sections: one is the electromagnetic processes through which hadrons gradually lose their kinetic energy, and the other is the hadronic scatterings which change the number of the hadronic particles.

Let us see what happens to a hadron injected into the thermal bath with the initial energy $E_{H_{i}}^{(\mathrm{in})}$ in more detail. Such a high energy hadron may be a direct decay product of $X$ or may be a daughter particle produced in the hadronic showers. As mentioned in the previous section, once a high energy hadron is injected into the thermal bath, it loses its energy down to $\sim \tilde{E}_{H_{i}}^{(R=1)}$ defined in Eq. (5.15). In our study, we approximate that the hadrons injected into the thermal bath with the initial energy $E_{H_{i}}^{(\text {in) }}$ scatters off the background nuclei (i.e., $p_{\mathrm{BG}}$ and $\alpha_{\mathrm{BG}}$ ) with the energy $\tilde{E}_{H_{i}}^{(R=1)}$ given above.

Since there are various hadronic processes, many possible final states exist even if we fix the initial-state particles. We specify the individual processes by denoting $(i, j ; k)$; here, $i$ and $j$ specify the projectile and target nuclei, respectively, while $k$ is for the final state. Then, we approximate the probability at which the projectile hadron $H_{i}$ scatters off a background nuclei via the process $(i, j ; k)$ as

$$
P_{(i, j ; k)}^{H_{i}}\left(E_{H_{i}}^{(\mathrm{in})} ; T\right) \equiv\left[\frac{n_{j}(T) \sigma_{(i, j ; k)}}{\sum_{m=p_{\mathrm{BG}}, \alpha_{\mathrm{BG}}} \sum n_{m}(T) \sigma_{(i, m ; l)}}\right]_{E_{H_{i}}=\tilde{E}_{H_{i}}^{(R=1)}}
$$

where $n_{j}$ is the number density of the background nucleus $j$, and $\sigma_{(i, j ; k)}$ is the cross section 
for the process $(i, j ; k)$ as a function of $E_{H_{i}}^{(\mathrm{in})}$. \#13

For each processes, we need to calculate the energy distribution of the final-state nuclei. The relevant final-state nuclei are $n, p, \mathrm{D}, \mathrm{T},{ }^{3} \mathrm{He}$, and ${ }^{4} \mathrm{He}$ in this case. ${ }^{\# 14}$ If we consider the scattering process $(i, j ; k)$ in which projectile hadron $H_{i}$ hits the target nucleon and produces the final-state hadron $H_{k}$, the energy distribution of $H_{k}$ is given by

$$
f_{H_{k}}^{(i, j ; k)}\left(E_{H_{i}}, E_{H_{k}}\right)=g_{H_{k}}^{(i, j ; k)} \frac{1}{\sigma_{(i, j ; k)}} \frac{d \sigma_{(i, j ; k)}}{d E_{H_{k}}}
$$

where $E_{H_{i}}$ here is the energy of $H_{i}$ at the time of the scattering while $E_{H_{k}}$ is the energy of $H_{k}$ just after being produced by the scattering process. In addition, $g_{H_{k}}^{(i, j ; k)}$ is the number of $H_{k}$ produced in the process $(i, j ; k)$. (For more details, see Appendix [C) Using $f_{H_{k}}^{(i, j ; k)}$, we can also obtain the total energy distribution of $H_{k}$ after the hadronic scattering of $H_{i}$, which is given by

$$
G_{H_{i} \rightarrow H_{k}}\left(E_{H_{i}}^{(\mathrm{in})}, E_{H_{k}} ; T\right)=\sum_{j=p, \alpha} \sum_{k} P_{(i, j ; k)}^{H_{i}}\left(E_{H_{i}}^{(\mathrm{in})} ; T\right) f_{H_{k}}^{(i, j ; k)}\left(\tilde{E}_{H_{i}}^{(R=1)}, E_{H_{k}}\right) .
$$

Notice that, if the initial-state particle has some energy distribution $F_{H_{i}}\left(E_{H_{i}} ; T\right.$ ) (where $E_{H_{i}}^{(\text {in) }}$ is the energy of $H_{i}$ just after being produced), then the distribution of the final-state particle $H_{k}$ is obtained as

$$
F_{H_{k}}^{\prime}\left(E_{H_{k}} ; T\right)=\sum_{i} \int d E_{H_{i}}^{(\mathrm{in})} F_{H_{i}}\left(E_{H_{i}}^{(\mathrm{in})} ; T\right) G_{H_{i} \rightarrow H_{k}}\left(E_{H_{i}}^{(\mathrm{in})}, E_{H_{k}} ; T\right) .
$$

With the relations given above, we can recursively follow the evolution of the hadronic shower. To make some image, let us consider the hadronic shower induced by a primary energetic nucleon $H_{i}^{(0)}$ generated by the decay of $X$. (To the primary nucleon, we assign the generation number "0.") We denote the initial energy of the primary nucleon as $E_{H_{i}^{(0)}}^{(\mathrm{in})}$. As mentioned, emitted nucleon loses its energy via the electromagnetic processes typically down to $\tilde{E}_{H_{i}^{(0)}}^{(R=1)}$, which satisfies $R^{H_{i}}\left(E_{H_{i}^{(0)}}^{(\mathrm{in})}, \tilde{E}_{H_{i}^{(0)}}^{(R=1)} ; T\right)=1$. Then, $H_{i}^{(0)}$ scatters of the background nuclei $\left(p_{\mathrm{BG}}\right.$ or $\left.\alpha_{\mathrm{BG}}\right)$ via the hadronic interactions. We call this "primary scattering." In our analysis, the primary scattering occurs with the energy $\tilde{E}_{H_{i}^{(0)}}^{(R=1)}$, and we obtain the energy distribution of the "1st generation" hadrons $H_{k}^{(1)}$ as $G_{H_{i}^{(0)} \rightarrow H_{k}^{(1)}}\left(E_{H_{i}^{(0)}}^{(\mathrm{in})}, E_{H_{k}^{(1)}} ; T\right)$. (Here and hereafter, the superscript for the hadronic species are to identify their generation number in the evolution of the hadronic shower.) Thus, once the energy distribution of the primary hadrons $F_{H_{i}}^{(0)}$ are known, we can calculate the \#13Notice that the probability $P_{(i, j ; k)}^{H_{i}}$ depends not only on $E_{H_{i}}^{(\mathrm{in})}$ and $T$ but also on the ${ }^{4} \mathrm{He}$ mass fraction $Y$. Such $Y$-dependence of $P_{(i, j ; k)}^{H_{i}}$ is taken into account in the numerical calculations.

\#14 In the hadronic scattering processes discussed so far, Li and Be are not produced. Treatment of those nuclei will be discussed in the next section. 
distribution of the first-generation hadrons by using the relation

$$
F_{H_{j}}^{(1)}\left(E_{H_{j}} ; T\right)=\sum_{i} \int d E_{H_{i}}^{(\mathrm{in})} F_{H_{i}}^{(0)}\left(E_{H_{i}}^{(\mathrm{in})} ; T\right) G_{H_{i} \rightarrow H_{k}}\left(E_{H_{i}}^{(\mathrm{in})}, E_{H_{j}} ; T\right) .
$$

In our study, the energy distributions of the primary (i.e., "0-th generation") hadrons are calculated by using the JETSET 7.4 Monte Carlo event generator. Similarly, the distribution functions for the $l$-th generation nuclei are recursively calculated by using the following relation:

$$
F_{H_{k}}^{(l)}\left(E_{H_{k}} ; T\right)=\sum_{i} \int d E_{H_{i}} F_{H_{i}}^{(l-1)}\left(E_{H_{i}} ; T\right) G_{H_{i} \rightarrow H_{k}}\left(E_{H_{i}}, E_{H_{k}} ; T\right),
$$

where $F_{H_{j}}^{(l)}$ is the distribution function of $H_{k}$ in $l$-th generation. After a large number of multiple scatterings, energy distribution function of any hadrons for energy region above the threshold energies of the hadrodissociation processes becomes negligibly small and the hadrodissociation processes stop.

\subsection{Approximations}

Evolution of the hadronic shower can be in principle understood by recursive procedure discussed in the previous subsection. In the actual calculation, however, it is difficult to obtain the resultant distributions of the shower particles without any simplification and approximation. One reason is that the number of hadrons contributing to the hadronic shower is enormous so it is difficult to track all the energy-loss processes of those hadrons. In addition, for some of the hadronic processes, experimental data for the cross sections are not available. Thus, in this subsection, we explain how we solve the basic equations given in the previous subsection with some simplifications and approximations.

Our primary purpose is to obtain conservative constraints on the properties of $X$. By adopting reasonable experimental and theoretical errors, the SBBN scenario predicts abundances of the light elements consistent with the observations, as discussed in Section 2. Thus, the non-standard processes usually make the theoretical constraints inconsistent with the observations. In particular, if hadronic scattering processes with the background $\alpha_{\mathrm{BG}}$ occurs too much, D and/or ${ }^{3} \mathrm{He}$ are overproduced. In addition, non-thermal processes, which will be discussed in the next section, may overproduce ${ }^{6} \mathrm{Li}$.

Importantly, for some processes, we do not have enough experimental informations. For such cases, we adopt some approximation or assumption so that the numbers of $\mathrm{D},{ }^{3} \mathrm{He}$, and ${ }^{6} \mathrm{Li}$ produced by the non-standard processes are minimized, resulting in conservative constraints. Thus, we should note that, for some case, the resultant abundances of $\mathrm{D},{ }^{3} \mathrm{He}$, and ${ }^{6} \mathrm{Li}$ obtained from our calculations are their lower bounds.

First of all, as we mentioned before, we simplify the treatment of the target (background) nuclei by approximating that the energetic hadrons scatter off only the background proton or $\alpha_{\mathrm{BG}}$. This is because most of the background nuclei are in the form 


\begin{tabular}{lccc}
\hline \hline Process & $i=n$ & $i=p$ & reaction type \\
\hline$\left(i, p_{\mathrm{BG}} ; 1\right)$ & $n+p_{\mathrm{BG}} \rightarrow n+p$ & $p+p_{\mathrm{BG}} \rightarrow p+p$ & elastic \\
$\left(i, p_{\mathrm{BG}} ; 2\right)$ & $n+p_{\mathrm{BG}} \rightarrow n+p+\pi$ & $p+p_{\mathrm{BG}} \rightarrow p+p+\pi$ & inelastic \\
$\left(i, p_{\mathrm{BG}} ; 3\right)$ & $n+p_{\mathrm{BG}} \rightarrow n+n+\pi$ & $p+p_{\mathrm{BG}} \rightarrow p+n+\pi$ & inelastic \\
\hline \hline
\end{tabular}

Table 2: Hadronic processes with background proton $p_{\mathrm{BG}}$

\begin{tabular}{lccc}
\hline \hline Process & $i=n$ & $i=p$ & reaction type \\
\hline$(i, \alpha ; 1)$ & $n+\alpha_{\mathrm{BG}} \rightarrow n+\alpha$ & $p+\alpha_{\mathrm{BG}} \rightarrow p+\alpha$ & elastic \\
$(i, \alpha ; 2)$ & $n+\alpha_{\mathrm{BG}} \rightarrow \mathrm{D}+\mathrm{T}$ & $p+\alpha_{\mathrm{BG}} \rightarrow \mathrm{D}+{ }^{3} \mathrm{He}$ & inelastic \\
$(i, \alpha ; 3)$ & $n+\alpha_{\mathrm{BG}} \rightarrow 2 n+{ }^{3} \mathrm{He}$ & $p+\alpha_{\mathrm{BG}} \rightarrow p+n+{ }^{3} \mathrm{He}$ & inelastic \\
$(i, \alpha ; 4)$ & $n+\alpha \rightarrow p+n+\mathrm{T}$ & $p+\alpha \rightarrow 2 p+\mathrm{T}$ & inelastic \\
$(i, \alpha ; 5)$ & $n+\alpha_{\mathrm{BG}} \rightarrow n+2 \mathrm{D}$ & $p+\alpha_{\mathrm{BG}} \rightarrow p+2 \mathrm{D}$ & inelastic \\
$(i, \alpha ; 6)$ & $n+\alpha_{\mathrm{BG}} \rightarrow p+2 n+\mathrm{D}$ & $p+\alpha_{\mathrm{BG}} \rightarrow 2 p+n+\mathrm{D}$ & inelastic \\
$(i, \alpha ; 7)$ & $n+\alpha_{\mathrm{BG}} \rightarrow 2 p+3 n$ & $p+\alpha_{\mathrm{BG}} \rightarrow 3 p+2 n$ & inelastic \\
$(i, \alpha ; 8)$ & $n+\alpha_{\mathrm{BG}} \rightarrow n+\alpha+\pi$ & $p+\alpha_{\mathrm{BG}} \rightarrow p+\alpha+\pi$ & inelastic \\
\hline \hline
\end{tabular}

Table 3: Hadronic processes with background $\alpha_{\mathrm{BG}}$

of the proton or ${ }^{4} \mathrm{He}$. Of course, some of the energetic hadrons may scatter off other light elements in the background which may be destructed by those processes. If such processes are effective, however, production of those light elements from the dissociation of the background $\alpha_{\mathrm{BG}}$ is far more important since $\alpha_{\mathrm{BG}}$ is more abundant than other light elements (except $p_{\mathrm{BG}}$ ). Thus, our constraints is not affected by our approximation on the target particles of the hadronic processes.

Second simplification is that, among various hadrons generated by hadronic scattering processes, only $p$ and $n$ are used as projectile nuclei in the next-step hadronic process (except for the non-thermal production processes of $\mathrm{Li}$ and $\mathrm{Be}$, which will be discussed in the next section). This is because most of the nuclei produced in the shower processes are $p$ or $n$, and also because we could not find sufficient experimental data for the cross sections for other nuclei. One might worry about the effects of the energetic ${ }^{4} \mathrm{He}$. However, energetic ${ }^{4} \mathrm{He}$ is much rarer than $p$ or $n$ in the hadronic shower since the energy transfer to ${ }^{4} \mathrm{He}$ in the elastic $p \alpha_{\mathrm{BG}}$ scattering process is fairly small. Notice also that the cross sections for the inelastic $p+\alpha_{\mathrm{BG}} \rightarrow p+\alpha+\cdots$ reactions are relatively small. Thus, the energetic ${ }^{4} \mathrm{He}$ has small effect on the evolution of the hadronic shower.

The hadronic scattering processes considered in our study are listed in Tables 2 and 3 , The experimental data of the cross sections are summarized in Refs. [66, 69. In addition, in our Monte Carlo analysis, we adopt $20 \%$ errors to all the hadronic cross sections.

In some case, we could not find sufficient experimental data and adopt some reasonable approximations or assumptions. In particular, the hadronic cross sections for the energy of 
the projectile higher than $\sim 20 \mathrm{GeV}$ cannot be found except for some $p p$ and $n p$ reactions. Fortunately, according to the existing data, however, the cross sections for the $p p$ and $n p$ reactions are known to become approximately constant at high-energy region [66]. Thus, in our analysis, the inelastic cross section for the $p \alpha$ process are assumed to be constant above $E>20 \mathrm{GeV}$. Our results are insensitive to this assumption since the evolution of the hadronic shower is mostly determined by the hadrons with energy less than $\sim \mathcal{O}(1)$ $\mathrm{GeV}$. In addition, sufficient experimental data are not available for the $n \alpha$ reactions. For these processes, we use the $\mathrm{SU}(2)$ isospin symmetry and use the cross sections of $p \alpha$ reactions for the $n \alpha$ reactions. Those cross section differ due to the Coulomb corrections. Using the familiar formula of the Coulomb correction factor [70, however, the Coulomb correction is estimated to be less than a few percent for the projectile energy larger than the threshold energy for the inelastic $n \alpha$ scattering process $(\sim 25 \mathrm{MeV})$. Thus, we neglect such a Coulomb correction.

In addition, the experimental data of the hadronic scattering processes for other processes are also insufficient. Thus, we make the following approximations for the daughter nuclei D, T, ${ }^{3} \mathrm{He}$, and ${ }^{4} \mathrm{He}$.

(i) In considering the hadronic process, the energetic daughter nuclei scatter off only the background proton and $\alpha_{\mathrm{BG}}$.

(ii) The daughter nucleus $A_{k}$ is assumed to survive only if (a) its typical energy just before the hadronic scattering (i.e., $\tilde{E}_{A_{k}}^{(R=1)}$ ) is smaller than the threshold energy for the dissociation of $A_{k}$ by scattering off the background proton, and (b) typical energy of the background photon in the center-of-mass frame is smaller than the threshold energy for the photodissociation process of $A_{k}$. (In fact, the second condition is not crucial; the resultant constraints on $X$ do not change much even if we do not include the condition (b).) That is, the surviving probability of the daughter particle $A_{k}\left(=\mathrm{D}, \mathrm{T},{ }^{3} \mathrm{He}\right.$ or $\left.{ }^{4} \mathrm{He}\right)$ is simply given by

$$
P_{A_{k} \rightarrow A_{k}}\left(E_{A_{k}} ; T\right)=\left\{\begin{array}{lll}
0 & : & \tilde{E}_{A_{k}}^{(R=1)}>E_{A_{k}}^{(\mathrm{th}, p)} \text { or } \sqrt{3 T E_{A_{k}}}>Q_{A_{k}} \\
1 & : & \text { otherwise }
\end{array}\right.
$$

where $E_{A_{k}}^{(\mathrm{th}, p)}$ is the threshold energy for the hadrodissociation process, while $Q_{A_{k}}$ is the binding energy of $A_{k}$.

(iii) The daughter nucleus $A_{k}$ is completely destroyed into energetic nucleons if $P_{A_{k} \rightarrow A_{k}}=$ 0. (For e.g., ${ }^{4} \mathrm{He}+p_{\mathrm{BG}} \rightarrow 3 p+2 n$.)

The approximation (i) is quite reasonable since almost all the baryons in the universe at the epoch we are interested are in the form of the proton or $\alpha_{\mathrm{BG}}$. The assumptions (ii) and (iii) are justified since our purpose is to obtain conservative constraints; indeed, the numbers of the non-thermally produced $\mathrm{D}, \mathrm{T},{ }^{3} \mathrm{He}$ and ${ }^{4} \mathrm{He}$ are underestimated with these approximations since the energetic nucleons produced by the dissociation of the light elements rarely dissociate $\alpha_{\mathrm{BG}}$ to produce other light elements. 
Since the target particle is $p_{\mathrm{BG}}$ or $\alpha_{\mathrm{BG}}$, we can rewrite $P_{(i, j ; k)}^{N_{i}}$ given in Eq. (7.1) as

$$
P_{(i, j ; k)}^{N_{i}}\left(E_{N_{i}}^{(\mathrm{in})} ; T\right)=\frac{n_{A_{j}}(T) \sigma_{(i, j ; k)}\left(\tilde{E}_{N_{i}}^{(R=1)}\right)}{n_{p}(T) \sigma_{N_{i} p}^{(\mathrm{tot})}\left(\tilde{E}_{N_{i}}^{(R=1)}\right)+n_{\alpha}(T) \sigma_{N_{i} \alpha}^{(\mathrm{tot})}\left(\tilde{E}_{N_{i}}^{(R=1)}\right)},
$$

where $\sigma_{N_{i} p}^{\text {(tot) }}$ and $\sigma_{N_{i} \alpha}^{\text {(tot) }}$ are total cross sections for the $N_{i} p_{\mathrm{BG}}$ and $N_{i} \alpha_{\mathrm{BG}}$ scattering processes, respectively. In our numerical calculations, distributions of the final-state hadrons (in particular, those of the light-elements) are calculated with this formula.

Since most of the final-state energetic hadrons are proton and neutron (as well as light mesons), we adopt an approximation such that only $p$ and $n$ are used as the initial-state energetic particles which cause hadrodissociations of $\alpha_{\mathrm{BG}}$. With such approximation, it is rather convenient to assign the generation numbers only to $p$ and $n$ by "integrating out" effects of other nuclei; using the distribution given in Eq. (7.3), we define the distribution of the $p$ and $n$ after taking account of the effects of other light elements as

$$
\begin{aligned}
\tilde{G}_{N \rightarrow N^{\prime}}\left(E_{N}^{(\mathrm{in})}, E_{N^{\prime}} ; T\right)= & G_{N \rightarrow N^{\prime}}\left(E_{N}^{(\mathrm{in})}, E_{N^{\prime}} ; T\right) \\
& +\sum_{A_{k} \neq p, n} \int d E_{A_{k}} G_{N \rightarrow A_{k}}\left(E_{N}^{(\mathrm{in})}, E_{A_{k}} ; T\right) P_{\left(A_{k}+\cdots \rightarrow N^{\prime}+\cdots\right)}^{A_{k}}\left(E_{A_{k}} ; T\right) \\
& g_{N^{\prime}}^{\left(A_{k}+\cdots \rightarrow N^{\prime}+\cdots\right)}\left(\tilde{E}_{A_{k}}^{(R=1)}, E_{N^{\prime}}\right),
\end{aligned}
$$

where $N$ and $N^{\prime}$ correspond to $p$ and $n$, and $g_{N^{\prime}}^{\left(A_{k}+\cdots \rightarrow N^{\prime}+\cdots\right)}\left(\tilde{E}_{A_{k}}^{(R=1)}, E_{N^{\prime}}\right)$ is the energy distribution of $N^{\prime}$ in the reaction $A_{k}+\cdots \rightarrow N^{\prime}+\cdots$ with the energy of $A_{k}$ being $\tilde{E}_{A_{k}}^{(R=1)}$. Although we have included the second term in Eq. (7.9), which take account of the effects of the nucleons generated from the secondary destruction of the non-thermally produced light elements, it is much smaller than the first term. Indeed, for one hadronic decay of $X$, numbers of $p$ and $n$ produced in the shower processes are of $\mathcal{O}(10-100)$ while the numbers of the destructed light elements are a few or less. Thus, our result is in fact insensitive to the approximation (iii) mentioned above. Furthermore, for other light elements $A_{j}=\mathrm{D}$, $\mathrm{T},{ }^{3} \mathrm{He}$, and ${ }^{4} \mathrm{He}$, we define

$$
\tilde{G}_{N \rightarrow A_{j}}\left(E_{N}^{(\mathrm{in})}, E_{A_{j}} ; T\right)=G_{N \rightarrow A_{j}}\left(E_{N}^{(\mathrm{in})}, E_{A_{j}} ; T\right) P_{A_{j} \rightarrow A_{j}}\left(E_{A_{j}} ; T\right) .
$$

Then, we define the distribution function of the light element $A_{j}$ after the hadronic scattering of $l$-th generation nucleons, which we denote $\tilde{F}_{A_{j}}$. Notice that $\tilde{F}_{A_{j}}$ obeys the recursion relation similar to Eq. (7.6):

$$
\tilde{F}_{A_{j}}^{(l)}\left(E_{A_{j}} ; T\right)=\sum_{N=p, n} \int d E_{N} \tilde{F}_{N}^{(l-1)}\left(E_{N} ; T\right) \tilde{G}_{N \rightarrow A_{j}}\left(E_{N}, E_{A_{j}} ; T\right)
$$

where, in the above expression, $A_{j}$ denotes all the possible light elements.

In our numerical analysis, scattering and energy-loss processes of the energetic nuclei are studied by using $\tilde{G}_{N \rightarrow N^{\prime}}$ and $\tilde{G}_{A_{i} \rightarrow A_{j}}$ defined above. (Details of the methods of our numerical calculation is explained in Appendix D.) 
With the distribution function given above, we calculate the number of produced or destructed nuclei by the decay of $X$. In our calculation, target of the energetic hadrons is $p_{\mathrm{BG}}$ or $\alpha_{\mathrm{BG}}$. In this case, numbers of the $h=\mathrm{D}, \mathrm{T}$ and ${ }^{3} \mathrm{He}$ are always increased by the decay of $X$ (as far as we neglect the subsequent thermal processes). Then, with the distribution function, we calculate the total number of the nuclei $A_{j}=\mathrm{D}, \mathrm{T}$ and ${ }^{3} \mathrm{He}$ produced by the hadronic decay of one $X$ :

$$
\begin{aligned}
\xi_{A_{j}}(T) & =\sum_{l=1}^{\infty} \int d E_{A_{j}} \tilde{F}_{A_{j}}^{(l)}\left(E_{A_{j}} ; T\right) \\
& =\int d E_{A_{j}} \int d E_{N} \sum_{N=n, p} \tilde{S}_{N}\left(E_{N} ; T\right) \tilde{G}_{N \rightarrow A_{j}}\left(E_{N}, A_{j} ; T\right)
\end{aligned}
$$

where

$$
\tilde{S}_{N}\left(E_{N} ; T\right) \equiv \sum_{l=0}^{\infty} \tilde{F}_{N}^{(l)}\left(E_{N} ; T\right) .
$$

We also calculate the total number of destroyed ${ }^{4} \mathrm{He}$ as

$$
\begin{aligned}
& \xi_{\alpha}(T)=\sum_{N=n, p} \int d E_{N} \tilde{S}_{N}\left(E_{N} ; T\right) \\
& {\left[\sum_{k=2}^{7} P_{(N, \alpha ; k)}^{N}\left(E_{N} ; T\right)+\int d E_{{ }^{4} \mathrm{He}} \tilde{G}_{N \rightarrow{ }^{4} \mathrm{He}}\left(E_{N}, E_{{ }^{4} \mathrm{He}} ; T\right)\left\{1-P_{{ }^{4} \mathrm{He} \rightarrow{ }^{4} \mathrm{He}}\left(E_{{ }^{4} \mathrm{He}} ; T\right)\right\}\right] .}
\end{aligned}
$$

We found that the hadrodissociation of the ${ }^{4} \mathrm{He}$ is dominated by the direct destruction in the hadronic process. Thus, although we have included the effects of the secondary destruction of the ${ }^{4} \mathrm{He}$ which is made energetic via the first hadronic scattering processes (i.e., the second term in Eq. (7.14)), such effect is subdominant and does not significantly change the constraints.

We should also consider the effects of the low-energy neutrons produced in the hadronic showers. Such neutrons may be energetic when they are produced, but they lose their energy as they propagate in the thermal bath. (The energetic neutrons mainly scatter off the background $p$ and $\alpha_{\mathrm{BG}}$.) Once the energy of the neutron becomes lower than the threshold energy of the destruction processes of the background $\alpha_{\mathrm{BG}}\left(E_{n \alpha}^{(\text {th })} \sim 25 \mathrm{MeV}\right)$, it no longer destruct the background $\alpha_{\mathrm{BG}}$. However, even after being thermalized, such extra-produced $n$ may affect the abundances of the light elements. In particular, $p$ may capture such lowenergy $n$ and $D$ may be produced. Furthermore, ${ }^{7}$ Be may be dissociated by the thermal neutron via the process ${ }^{7} \mathrm{Be}\left(n,{ }^{3} \mathrm{He}\right){ }^{4} \mathrm{He}$, which reduces the resultant abundance of ${ }^{7} \mathrm{Li}$. These processes are included in our BBN code. Importantly, at high enough temperature, the neutron with energy lower than $E_{n \alpha}^{(\text {th })}$ does not decay before being thermalized since its lifetime is much longer than the thermalization time. Thus, effects of the low-energy 


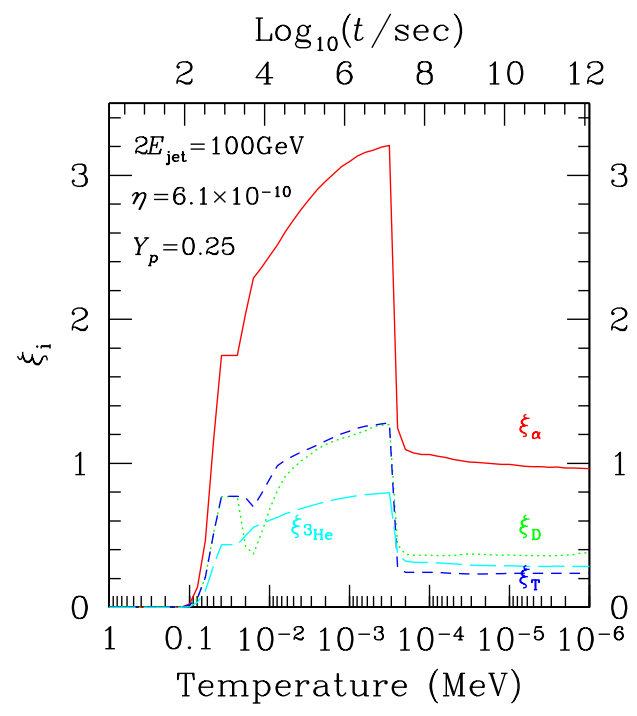

Figure 24: Number of produced or destructed hadrons per one hadronic decay of $X$ with $m_{X}=100 \mathrm{GeV} . \xi_{\alpha}$ (solid line) is the number of the destructed ${ }^{4}$ He while $\xi_{\mathrm{D}}$ (dotted line), $\xi_{\mathrm{T}}$ (dashed line), and $\xi_{3} \mathrm{He}$ (long dashed line) are the number of $\mathrm{D}, \mathrm{T}$ and ${ }^{3} \mathrm{He}$ produced by $X$-decay, respectively. We take $Y_{p}=0.25 \eta=6.1 \times 10^{-10}$, and $2 E_{\text {jet }}=m_{X}$.

neutron is taken into account by injecting thermal neutron into the thermal bath. The number of the neutrons produced by the hadronic decay of $X$ as

$$
\xi_{n}(T)=\int d E_{n} \tilde{F}_{n}^{(\infty)}\left(E_{n} ; T\right) .
$$

In Figs. 24, 25 and 26, we plot $\xi_{\mathrm{D}}, \xi_{\mathrm{T}}, \xi^{3} \mathrm{He}$ and $\xi_{\alpha}$ as functions of the temperature $T$ for $m_{X}=100 \mathrm{GeV}, 1 \mathrm{TeV}$ and $10 \mathrm{TeV}$. As one can see, the $\xi$-parameters almost vanishes at $T \gtrsim 0.1 \mathrm{MeV}$. This is because, for such high temperature, energetic hadrons are stopped by the electromagnetic processes before scattering off the background nuclei. Thus, in this case, hadrodissociation processes do not change the abundances of the light elements. As the temperature becomes lower, the $\xi$-parameters increases until $T \sim 0.3 \mathrm{keV}$. In this period, the hadrodissociation is dominated by the energetic neutron since the mean-free-path of the neutron is much longer than that of proton. Energy-loss of the neutron becomes less efficient as the temperature becomes lower, so the effects of the hadrodissociations become more effective as the temperature becomes lower. Once the cosmic temperature becomes lower than $T \sim 0.3 \mathrm{keV}$, however, the neutron decays before scattering off $\alpha_{\mathrm{BG}}$. Since the stopping process of the proton is more efficient than that of the neutron, hadrodissociation is suppressed at the low enough temperature. Thus, we see sharp drop-off of the $\xi$-parameters at $T \sim 0.3 \mathrm{keV}$.

We also plot $\xi_{n}$ in Fig. 27. Note that, in Fig. 27. we subtract the number of neutrons which are contained in the initial spectrum of neutrons $\tilde{F}_{n}^{(0)}$ from $\xi_{n}$ in order to show the 


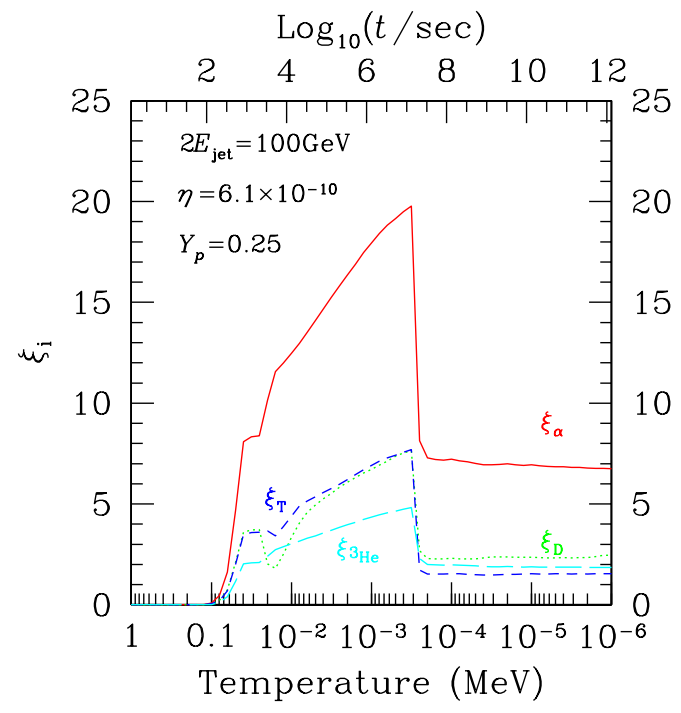

Figure 25: Same as Fig. 24, except for $m_{X}=1 \mathrm{TeV}$.

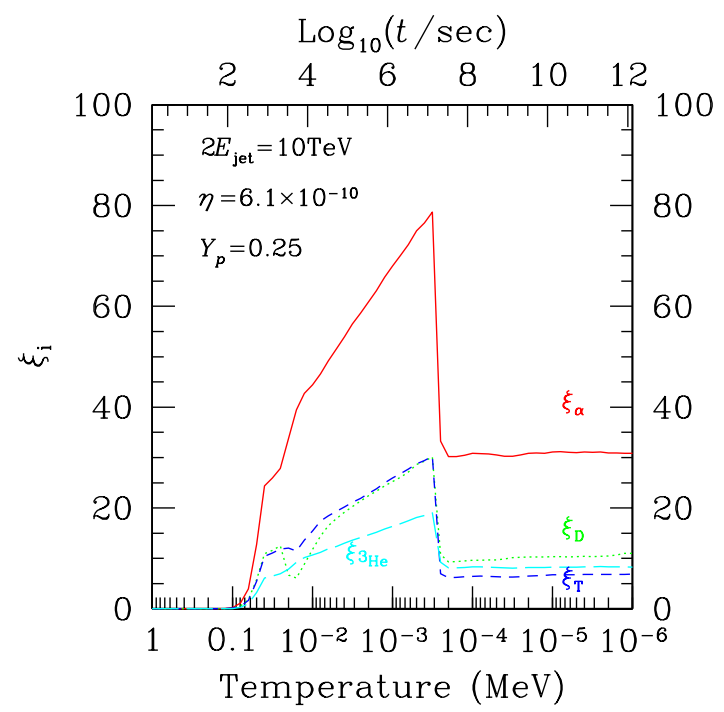

Figure 26: Same as Fig. 24, except for $m_{X}=10 \mathrm{TeV}$. 


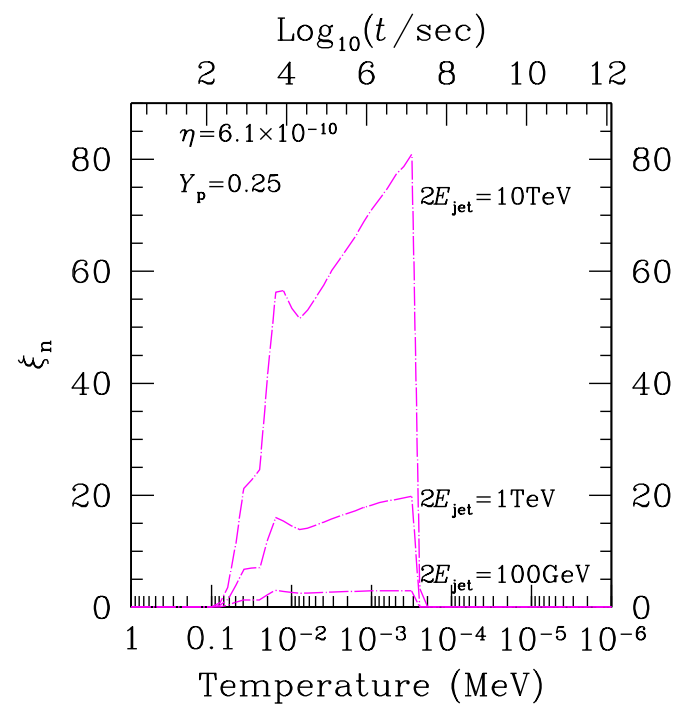

Figure 27: $\xi_{n}$ as a function of the temperature. Here, we take $Y_{p}=0.25$ and $\eta=6.1 \times 10^{-10}$, the total energy of the two hadronic jets is $2 E_{\text {jet }}=100 \mathrm{GeV}, 1 \mathrm{TeV}$ and $10 \mathrm{TeV}$.

number of the secondary produced neutrons. The drastic decrease at $T \sim 0.3 \mathrm{keV}$ is, again, due to the neutron decay.

\section{Non-thermal Production of Lithium and Beryllium}

In this section, we discuss the non-thermal production processes of $\mathrm{Li}$ and $\mathrm{Be}$. As we have discussed in the previous sections, energetic $\mathrm{T},{ }^{3} \mathrm{He}$ and ${ }^{4} \mathrm{He}$ can be produced by the hadronic or photodissociation processes with the background $\alpha_{\mathrm{BG}}$. Such energetic nuclei may scatter off the background $\alpha_{\mathrm{BG}}$ again and produce other nuclei, in particular, ${ }^{6} \mathrm{Li}$, ${ }^{7} \mathrm{Li}$, and ${ }^{7} \mathrm{Be}$. Although these collisions are not so frequent, they are important since the observations severely constrain the primordial abundances of ${ }^{6} \mathrm{Li}$ and ${ }^{7} \mathrm{Li}$.

First, we consider the non-thermal production of ${ }^{6} \mathrm{Li}$ by the energetic $\mathrm{T}$ and ${ }^{3} \mathrm{He}$. In this case, energetic $\mathrm{T}$ and ${ }^{3} \mathrm{He}$ are produced by

$$
p(n)+\alpha_{\mathrm{BG}} \rightarrow\left\{\begin{array}{l}
\mathrm{T}+\cdots \\
{ }^{3} \mathrm{He}+\cdots
\end{array},\right.
$$

and these $\mathrm{T}$ and ${ }^{3} \mathrm{He}$ scatter off the $\alpha_{\mathrm{BG}}$ to produce ${ }^{6} \mathrm{Li}$ :

$$
\begin{array}{r}
\mathrm{T}+\alpha_{\mathrm{BG}} \rightarrow{ }^{6} \mathrm{Li}+n, \\
{ }^{3} \mathrm{He}+\alpha_{\mathrm{BG}} \rightarrow{ }^{6} \mathrm{Li}+p .
\end{array}
$$




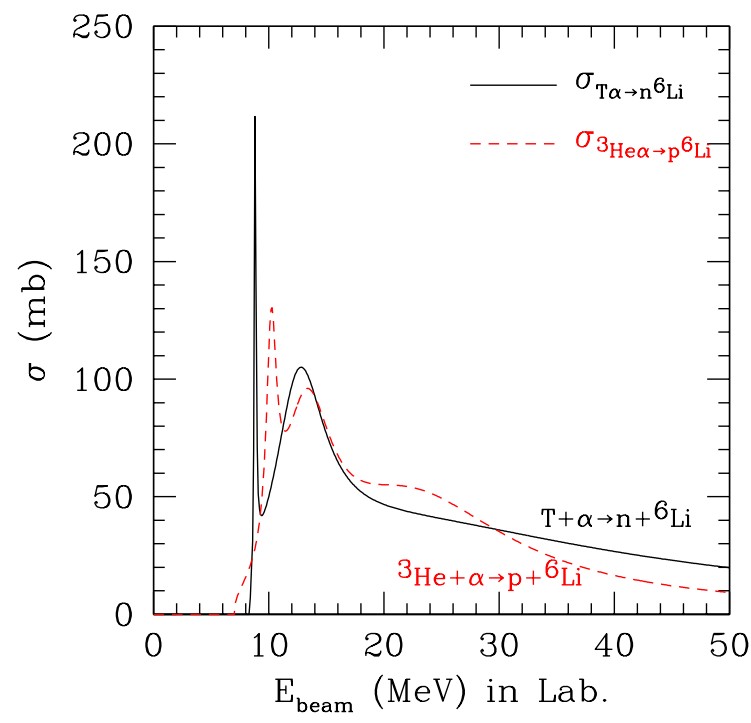

Figure 28: The cross sections of the ${ }^{6} \mathrm{Li}$ production processes. The solid (dashed) line is for $\mathrm{T}+{ }^{4} \mathrm{He} \rightarrow{ }^{6} \mathrm{Li}+n\left({ }^{3} \mathrm{He}+{ }^{4} \mathrm{He} \rightarrow{ }^{6} \mathrm{Li}+p\right)$.

Once the energetic nucleus $A_{i}=\mathrm{T}$ or ${ }^{3} \mathrm{He}$ is injected into the thermal bath, it loses its energy via the electromagnetic interactions by scattering of the background electron and photon while it also scatters off the background $\alpha_{\mathrm{BG}}$. With the energy-loss rate $\left(d E_{A_{i}} / d t\right)$ given in the previous section, number of ${ }^{6} \mathrm{Li}$ produced by one $A_{i}$ is

$$
\int_{\tilde{E}_{A_{i}}^{(R=1)}}^{E_{A_{i}}^{(\mathrm{in})}} d E_{A_{i}}\left(\frac{d E_{A_{i}}}{d t}\right)^{-1} n_{\alpha} \sigma_{A_{i}+\alpha_{\mathrm{BG}} \rightarrow{ }^{6} \mathrm{Li}+\cdots}\left(E_{A_{i}}\right) \beta_{A_{i}},
$$

where $E_{A_{i}}^{(\mathrm{in})}$ is the initial energy of $A_{i}, \beta_{A_{i}}$ is the velocity of $A_{i}$, and $\tilde{E}_{A_{i}}^{(R=1)}$ is the typical energy of $A_{i}$ just before its collision with background proton or $\alpha_{\mathrm{BG}}$ (see Section 5). In addition, $\sigma_{A_{i}+\alpha_{\mathrm{BG}} \rightarrow{ }^{6} \mathrm{Li}+\cdots}$ is the cross section of the process (8.2) or (8.3). (We plot the experimental data of them in Fig. 28, [11, 172, 16.) Summing up the contributions of T and ${ }^{3} \mathrm{He}$, the number of ${ }^{6} \mathrm{Li}$ produced by the above process per one hadronic decay of $X$ is given by

$$
\begin{aligned}
\xi_{6 \mathrm{Li}}^{\left(\mathrm{T},{ }^{3} \mathrm{He}\right)}= & \sum_{A_{i}=\mathrm{T},{ }^{3} \mathrm{He}} \int_{0}^{\infty} d E_{A_{i}}^{(\mathrm{in})} f_{A_{i}}\left(E_{A_{i}}^{(\mathrm{in})}\right) \\
& \int_{\tilde{E}_{A_{i}}^{(R=1)}}^{E_{A_{i}}^{(\mathrm{in})}} d E_{A_{i}}\left(\frac{d E_{A_{i}}}{d t}\right)^{-1} n_{\alpha} \sigma_{A_{i}+\alpha_{\mathrm{BG}} \rightarrow{ }^{6} \mathrm{Li}+\cdots}\left(E_{A_{i}}\right) \beta_{A_{i}} P_{6} \mathrm{Li} \rightarrow{ }^{6} \mathrm{Li}
\end{aligned}
$$

where $P^{{ }^{6} \mathrm{Li} \rightarrow{ }^{6} \mathrm{Li}}$ is the surviving rate of the non-thermally produced ${ }^{6} \mathrm{Li},{ }^{\# 15} f_{A_{i}}$ is the cumulative energy-distribution function of energetic $\mathrm{T}$ and ${ }^{3} \mathrm{He}$ during whole period of ${ }^{\# 15}$ In fact, non-thermally produced ${ }^{6} \mathrm{Li}$ can be also destroyed by the process ${ }^{6} \mathrm{Li}\left(p_{\mathrm{BG}},{ }^{4} \mathrm{He}\right){ }^{3} \mathrm{He}$ after being 


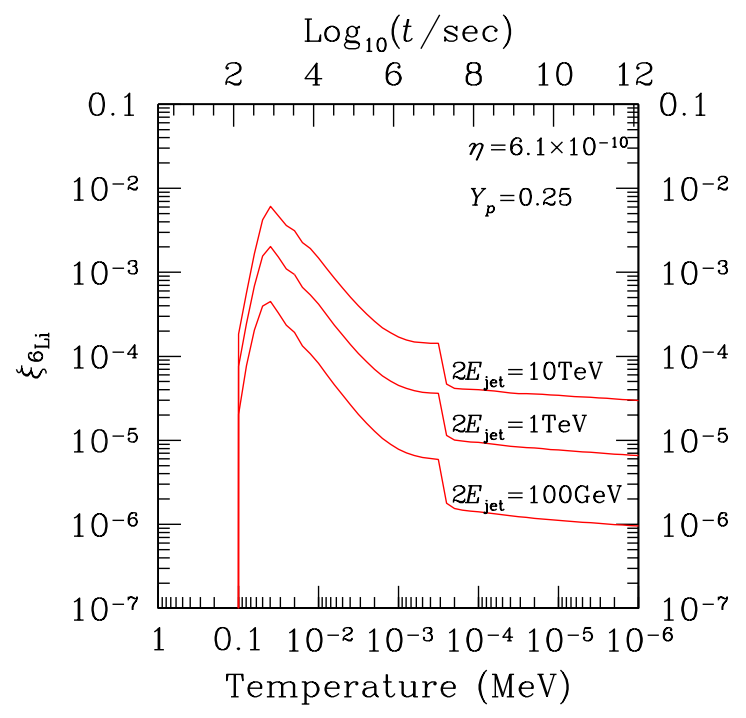

Figure 29: $\xi_{6 \mathrm{Li}}^{\left(\mathrm{T},{ }^{3} \mathrm{He}\right)}$ as a function of the temperature for $2 E_{\text {jet }}=100 \mathrm{GeV}, 1 \mathrm{TeV}$ and $10 \mathrm{TeV}$. Here, we take $Y_{p}=0.25$ and $\eta=6.1 \times 10^{-10}$.

the evolution of the hadronic shower. For the energy distribution of $\mathrm{T}$ and ${ }^{3} \mathrm{He}$ produced by the hadrodissociation processes, we use the experimental data [69] (see Appendix [C). Importantly, experimental result suggests that the energy distribution of the final-state $\mathrm{T}$ (and ${ }^{3} \mathrm{He}$ ) is almost independent of the energy of the initial state energetic neutron. Thus, we use the following formula for $f_{A_{i}}$ (with $A_{i}=\mathrm{T}$ and ${ }^{3} \mathrm{He}$ )

$$
f_{A_{i}}\left(E_{A_{i}}\right)=\frac{\xi_{A_{i}}}{\sigma_{N+\alpha \rightarrow A_{i}+\cdots}} \frac{d \sigma_{N+\alpha \rightarrow A_{i}+\cdots}}{d E_{A_{i}}}
$$

Fitting formula for the differential cross section obtained from the experimental data, which is used in our analysis, is given in Eq. (C.25).

The non-thermally produced ${ }^{6} \mathrm{Li}$ is still energetic with their kinetic energies of $\mathcal{O}(1-$ 10) $\mathrm{MeV}$ and might be destroyed by scattering off the background nuclei (in particular, protons) before it is thermalized. To estimate the number of ${ }^{6} \mathrm{Li}$ destroyed after the non-thermal production, we calculate the surviving probability $P^{{ }^{6} \mathrm{Li} \rightarrow{ }^{6} \mathrm{Li}}$. (For details, see Appendix[E]) Then, for the cosmic temperature we are interested in (i.e., $T \lesssim 100 \mathrm{keV}$ ) we have found that the surviving rate is very close to 1 . Thus, almost all the non-thermally produced ${ }^{6} \mathrm{Li}$ survive until being thermalized. (Same is true for ${ }^{6} \mathrm{Li},{ }^{7} \mathrm{Li},{ }^{7} \mathrm{Be}$ produced by the non-thermal processes with energetic ${ }^{4} \mathrm{He}$, which will be discussed below.)

Using the cumulative energy-distribution function obtained by following the evolution of the hadronic shower, we calculate the $\xi_{6}^{\left(\mathrm{T},{ }^{3} \mathrm{He}\right)}$ parameters for various background temthermalized. Such an effect is taken in account in the standard code of the BBN calculation we used [50], and hence is not included in $P_{6} \mathrm{Li} \rightarrow{ }^{6} \mathrm{Li}$. 


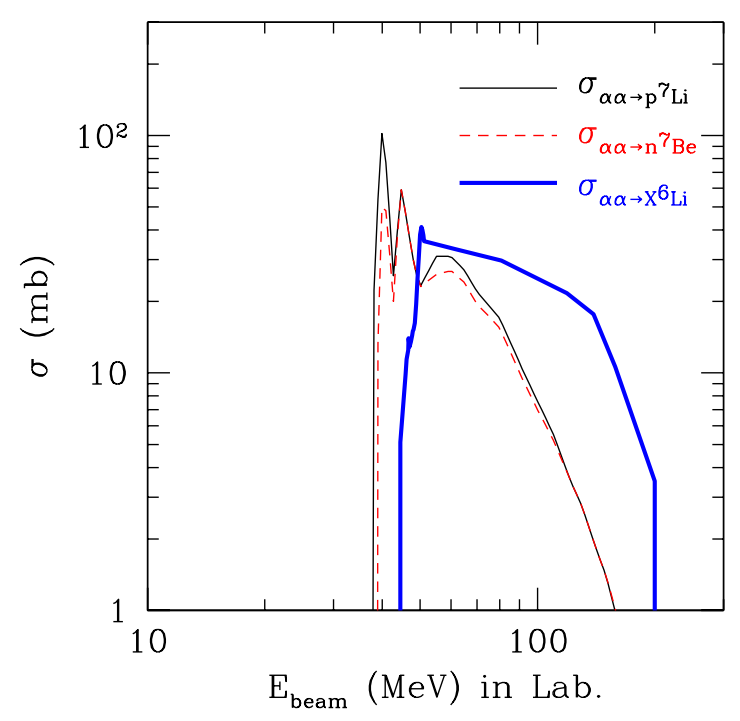

Figure 30: The cross sections of the ${ }^{6} \mathrm{Li},{ }^{7} \mathrm{Li}$ and ${ }^{7} \mathrm{Be}$ production process. The solid (dashed) line is for ${ }^{4} \mathrm{He}+{ }^{4} \mathrm{He} \rightarrow{ }^{7} \mathrm{Li}+p\left({ }^{3} \mathrm{He}+{ }^{4} \mathrm{He} \rightarrow{ }^{7} \mathrm{Be}+n\right)$, while thick solid line is the total cross section for the process ${ }^{4} \mathrm{He}+{ }^{4} \mathrm{He} \rightarrow{ }^{6} \mathrm{Li}+\cdots$.

peratures (as well as other cosmological and model parameters). The numerical result is shown in Fig. 20. As one can see, $\xi_{6 \mathrm{Li}}^{\left(\mathrm{T}^{3} \mathrm{He}\right)}$ is suppressed when $T \gtrsim 100 \mathrm{keV}$. This cut off is from the fact that, at such high temperature, all the hadronic particles are stopped by the electromagnetic processes before scattering off $\alpha_{\mathrm{BG}}$. (In addition, at high temperature, surviving rate of ${ }^{6} \mathrm{Li}$ is also suppressed.) In addition, we see a sharp drop-off of $\xi_{6 \mathrm{Li}}^{\left(\mathrm{T},{ }^{3} \mathrm{He}\right)}$ at $T \sim 0.3 \mathrm{keV}$, which is due to the decay of the neutron during the propagation in the universe.

Next we consider the non-thermal production of ${ }^{6} \mathrm{Li},{ }^{7} \mathrm{Li}$, and ${ }^{7} \mathrm{Be}$ through the collision of energetic ${ }^{4} \mathrm{He}$ with background $\alpha_{\mathrm{BG}}$. Such energetic ${ }^{4} \mathrm{He}$ is produced by the elastic and inelastic scatterings between high-energy nucleons and background $\alpha$. The number of the non-thermally produced nuclei per one decaying $X$ is expressed as

$$
\begin{aligned}
& \xi_{A_{k}}^{\left({ }^{4} \mathrm{He}\right)}=\int_{0}^{\infty} d E_{{ }^{4} \mathrm{He}}^{(\mathrm{in})} f_{{ }^{4} \mathrm{He}}\left(E_{4}^{(\mathrm{in})}\right)
\end{aligned}
$$

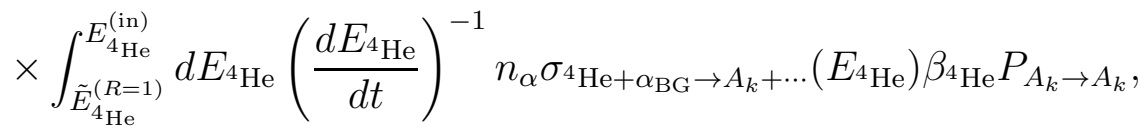

where, here, $A_{k}={ }^{6} \mathrm{Li},{ }^{7} \mathrm{Li}$, and ${ }^{7} \mathrm{Be}$. In Fig. 30 the experimental data of the differential cross sections for ${ }^{4} \mathrm{He}+{ }^{4} \mathrm{He} \rightarrow{ }^{7} \mathrm{Li}+p,{ }^{7} \mathrm{Be}+n$, and ${ }^{6} \mathrm{Li}+X$ are plotted [73, 74, 75, 72, As will be shown in Appendix [C, the elastic scattering of high-energy nucleons with $\alpha_{\mathrm{BG}}$ $\left(N+\alpha_{\mathrm{BG}} \rightarrow N+\alpha\right)$ is not important for these production processes of ${ }^{6} \mathrm{Li},{ }^{7} \mathrm{Li}$, and ${ }^{7} \mathrm{Be}$. That is because the transfered energy to ${ }^{4} \mathrm{He}$ in the elastic scattering is much smaller than 


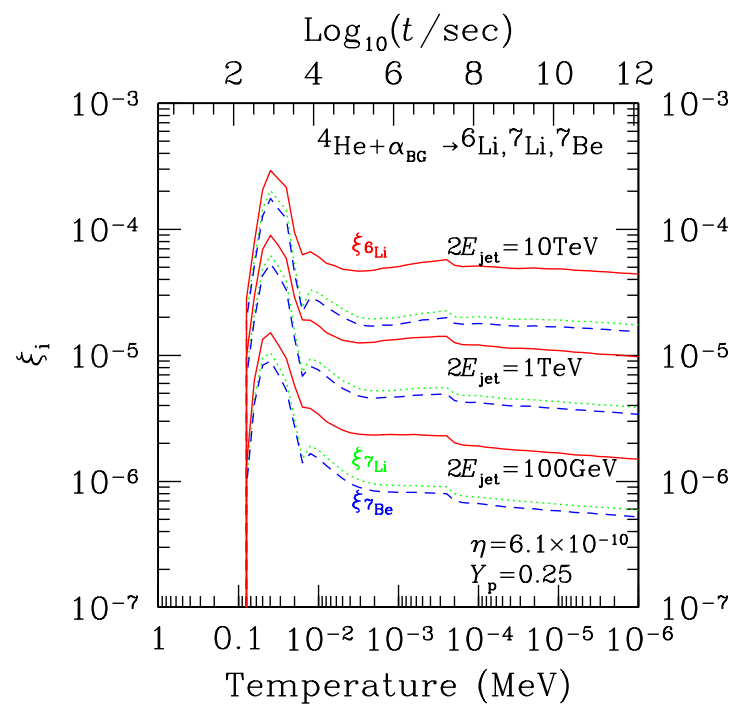

Figure 31: $\xi_{6 \mathrm{Li}}^{\left({ }^{4} \mathrm{He}\right)}, \xi_{7 \mathrm{Li}}^{\left({ }^{4} \mathrm{He}\right)}$, and $\xi_{7 \mathrm{Be}}^{\left({ }^{4} \mathrm{He}\right)}$ as functions of the temperature for $2 E_{\text {jet }}=100 \mathrm{GeV}$, $1 \mathrm{TeV}$ and $10 \mathrm{TeV}$. Here, we take $Y_{p}=0.25$ and $\eta=6.1 \times 10^{-10}$.

the case of the inelastic scattering $\left(N+\alpha_{\mathrm{BG}} \rightarrow N+\alpha+\pi^{\prime} s\right)$, although the cross section is fairly large.

Although the expressions (8.5) and (8.7) have the same structure, estimation of $\xi_{A_{k}}^{\left({ }^{4} \mathrm{He}\right)}$ is rather difficult. This is because we only have insufficient data for the transfered energies to ${ }^{4} \mathrm{He}$ in the non-elastic scattering processes to produce energetic ${ }^{4} \mathrm{He}$. In particular, for the process $p / n+{ }^{4} \mathrm{He} \rightarrow p / n+{ }^{4} \mathrm{He}+\pi+\cdots$, energy distribution of ${ }^{4} \mathrm{He}$ in the final state is quite uncertain at around the threshold energy of this scattering process. The number of the non-thermally produced $\mathrm{Li}$ and Be, however, depend on the energy distribution of ${ }^{4} \mathrm{He}$. At the present stage, we have to conclude that the reliable estimation of the number of non-thermally produced $\mathrm{Li}$ and Be from the process ${ }^{4} \mathrm{He}+\alpha_{\mathrm{BG}} \rightarrow \mathrm{Li} / \mathrm{Be}+\cdots$ is difficult. Thus, we will not include the non-thermally produced Li and Be from this class of processes when we derive the constraint on $X$.

We can, however, estimate the number of $\mathrm{Li}$ and Be produced from the process ${ }^{4} \mathrm{He}+$ $\alpha_{\mathrm{BG}} \rightarrow \mathrm{Li} / \mathrm{Be}+\cdots$ by adopting the energy distribution of ${ }^{4} \mathrm{He}$ generated from our shower algorithm (see Appendix C):

$$
f_{{ }^{4} \mathrm{He}}\left(E_{{ }^{4} \mathrm{He}}\right)=\sum_{N=p, n} \sum_{l=0}^{\infty} \int d E_{N} F^{(l)}\left(E_{N}\right) \tilde{G}_{N \rightarrow{ }^{4} \mathrm{He}}\left(E_{N}, E^{{ }^{4} \mathrm{He}}\right) .
$$

Using this relation, we estimate $\xi_{6}^{\left({ }^{4} \mathrm{He}\right)}, \xi_{7}^{\left({ }^{4} \mathrm{He}\right)}$, and $\xi_{7_{\mathrm{Be}}}^{\left({ }^{4} \mathrm{He}\right)}$. The results are shown in Fig. 31. The results indicate that, by comparing with Fig. 29, the effects of ${ }^{4} \mathrm{He}-\alpha_{\mathrm{BG}}$ collision are less significant than those of the T- $\alpha_{\mathrm{BG}}$ and ${ }^{3} \mathrm{He}-\alpha_{\mathrm{BG}}$ collisions. In addition, we have 
checked that, even if we adopt $\xi_{6}^{\left({ }^{4} \mathrm{He}\right)}, \xi_{7}^{\left({ }^{4} \mathrm{He}\right)}$, and $\xi_{{ }^{7} \mathrm{Be}}^{\left({ }^{4} \mathrm{He}\right)}$ obtained above, no significant change of the resultant constraints on $X$ is seen.

So far, we have discussed the case where the parent nucleus inducing the non-thermal production of $\mathrm{Li}$ and $\mathrm{Be}$ is produced by the hadronic scattering processes. Energetic nuclei are, in fact, also produced by the photodissociation processes of $\alpha_{\mathrm{BG}}$. Thus, now we consider the effects of the non-thermal production of $\mathrm{Li}$ and Be induced by the energetic nucleus generated by the photodissociation processes 13 .

Using energetic photons produced in the electromagnetic shower processes, energetic $\mathrm{T}$ and ${ }^{3} \mathrm{He}$ can be produced by the processes

$$
\gamma+\alpha_{\mathrm{BG}} \rightarrow\left\{\begin{array}{l}
\mathrm{T}+p \\
{ }^{3} \mathrm{He}+n
\end{array} .\right.
$$

Then, using $\mathrm{T}$ and ${ }^{3} \mathrm{He}$ produced by the above process, non-thermal production of ${ }^{6} \mathrm{Li}$ is possible with the process (8.2) and (8.3).

We take account of these effects including the following term in the Boltzmann equation:

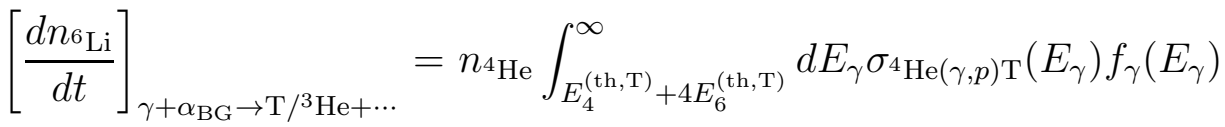

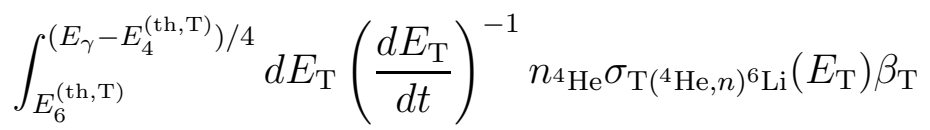

$$
\begin{aligned}
& +n^{4} \mathrm{He} \int_{E_{4}^{\left(\mathrm{th},{ }^{3} \mathrm{He}\right)}+4 E_{6}^{\left(\mathrm{th},{ }^{3} \mathrm{He}\right)}}^{\infty} d E_{\gamma} \sigma^{4} \mathrm{He}(\gamma, p)^{3} \mathrm{He}\left(E_{\gamma}\right) f_{\gamma}\left(E_{\gamma}\right)
\end{aligned}
$$

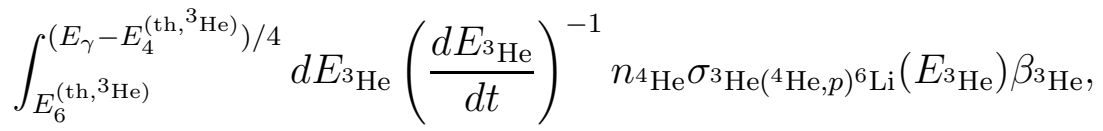

where $E_{4}^{(\mathrm{th}, \mathrm{T})}$ and $E_{6}^{(\mathrm{th}, \mathrm{T})}\left(E_{4}^{\left(\mathrm{th},{ }^{3} \mathrm{He}\right)}\right.$ and $\left.E_{6}^{\left(\mathrm{th},{ }^{3} \mathrm{He}\right)}\right)$ are threshold energies for the processes $\gamma+{ }^{4} \mathrm{He} \rightarrow \mathrm{T}+p$ and $\mathrm{T}+{ }^{4} \mathrm{He} \rightarrow{ }^{6} \mathrm{Li}+n\left(\gamma+{ }^{4} \mathrm{He} \rightarrow{ }^{3} \mathrm{He}+p\right.$ and ${ }^{3} \mathrm{He}+{ }^{4} \mathrm{He} \rightarrow{ }^{6} \mathrm{Li}+p$, respectively. \#16 In addition, $\sigma_{\mathrm{T}\left({ }^{4} \mathrm{He}, n\right)^{6} \mathrm{Li}}\left(E_{\mathrm{T}}\right)\left(\sigma^{\sigma_{3} \mathrm{He}\left({ }^{4} \mathrm{He}, p\right){ }^{6} \mathrm{Li}}\left(E^{3} \mathrm{He}\right)\right)$ is the cross section for the process $\mathrm{T}+{ }^{4} \mathrm{He} \rightarrow{ }^{6} \mathrm{Li}+n\left({ }^{3} \mathrm{He}+{ }^{4} \mathrm{He} \rightarrow{ }^{6} \mathrm{Li}+p\right)$ with $E_{\mathrm{T}}\left(E^{3} \mathrm{He}\right)$ being the energy of the injected $\mathrm{T}\left({ }^{3} \mathrm{He}\right)$. For these cross sections, we use the formula given in Ref. [16]; $E_{\mathrm{T}}$ dependence of $\sigma_{\mathrm{T}\left({ }^{4} \mathrm{He}, n\right){ }^{6} \mathrm{Li}}\left(E_{\mathrm{T}}\right)$ is shown in Fig. 28 .

\footnotetext{
\#16 Effects of the non-thermal production of ${ }^{6} \mathrm{Li}$ by $\mathrm{T}$ and ${ }^{3} \mathrm{He}$ produced by the photodissociations become important at relatively low temperature where $\tilde{E}_{6 \mathrm{Li}}^{(R=1)}$ becomes smaller than the threshold energies. Thus, in Eq. (8.9), the threshold energies of the ${ }^{6} \mathrm{Li}$ productions are used for the lower bound of the integration.
} 


\section{General Results}

\subsection{Outline}

In this section, we present our numerical results. In particular, we compare the theoretical predictions on the abundances of the light elements with the observations and derive constraints on the properties of $X$.

In our analysis, we first calculate the evolution of the number density of $X$ using

$$
\frac{d n_{X}}{d t}=-3 H n_{X}-\Gamma_{X} n_{X}
$$

At each temperature, photodissociation rates are calculated by numerically integrating the photon spectrum and the relevant cross sections. In addition, we also calculate the $\xi$-parameters defined in the previous sections.

Then, we obtain the Boltzmann equations for the light elements. Evolution of the nucleons $N(=p, n)$ is governed by

$$
\frac{d n_{N}}{d t}=\left[\frac{d n_{N}}{d t}\right]_{\mathrm{SBBN}}+\left[\frac{d n_{N}}{d t}\right]_{\text {photodis }}+B_{h} n_{X} \Gamma_{X} \xi_{N}+\left[\frac{d n_{N}}{d t}\right]_{\mathrm{IC}} .
$$

For other light elements with atomic number 2 or 3 (i.e., $A_{i}=\mathrm{D}, \mathrm{T},{ }^{3} \mathrm{He}$ ), we obtain

$$
\frac{d n_{A_{i}}}{d t}=\left[\frac{d n_{A_{i}}}{d t}\right]_{\mathrm{SBBN}}+\left[\frac{d n_{A_{i}}}{d t}\right]_{\text {photodis }}+B_{h} n_{X} \Gamma_{X} \xi_{A_{i}}
$$

while for ${ }^{4} \mathrm{He}$,

$$
\frac{d n^{4} \mathrm{He}}{d t}=\left[\frac{d n^{4} \mathrm{He}}{d t}\right]_{\mathrm{SBBN}}+\left[\frac{d n^{4} \mathrm{He}}{d t}\right]_{\text {photodis }}-B_{h} n_{X} \Gamma_{X} \xi_{\alpha}
$$

For ${ }^{6} \mathrm{Li}$, we include the non-thermal secondary production process discussed in the previous section and hence we obtain

$$
\left.\frac{d n^{6} \mathrm{Li}}{d t}=\left[\frac{d n_{6} \mathrm{Li}}{d t}\right]_{\mathrm{SBBN}}+\left[\frac{d n_{6} \mathrm{Li}}{d t}\right]_{\text {photodis }}+\left[\frac{d n_{6} \mathrm{Li}}{d t}\right]_{\gamma+\alpha_{\mathrm{BG}} \rightarrow \mathrm{T} /{ }^{3} \mathrm{He}+\cdots}+B_{h} n_{X} \Gamma_{X} \xi^{(\mathrm{T} \mathrm{Li}}{ }^{3} \mathrm{He}\right) .
$$

Here, the terms with the subscript "SBBN" represent the SBBN contributions to the Boltzmann equations (including the effect of the cosmic expansion).

In order to solve these equations, we have modified the Kawano Code [50] to include the effects of the non-thermal processes. Then, we numerically solve the above Boltzmann equations (as well as the evolutions of other cosmological quantities) and calculate the light-element abundances. 


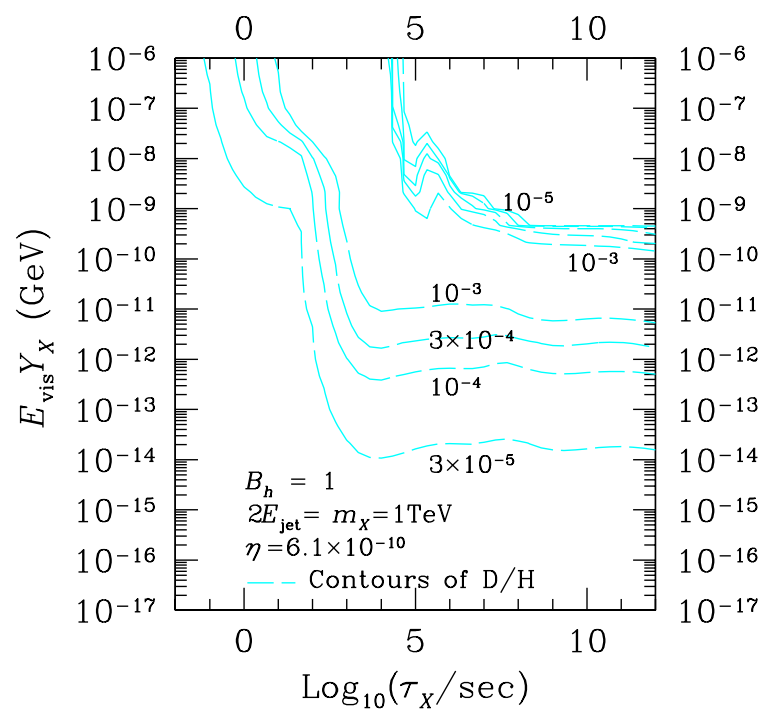

Figure 32: Contours of constant D/H on the $\tau_{X}$ vs. $E_{\mathrm{vis}} Y_{X}$ plane for $m_{X}=1 \mathrm{TeV}$. Here we take $B_{h}=1, E_{\text {vis }}=m_{X}$, and $X$ is assume to decay into two hadronic jets with $2 E_{\text {jet }}=m_{X}$. Here, we take $\eta=6.1 \times 10^{-10}$. In the $\mathrm{SBBN}$, the theoreical predication is $(\mathrm{D} / \mathrm{H})_{\mathrm{SBBN}}=2.78 \times 10^{-5}$.

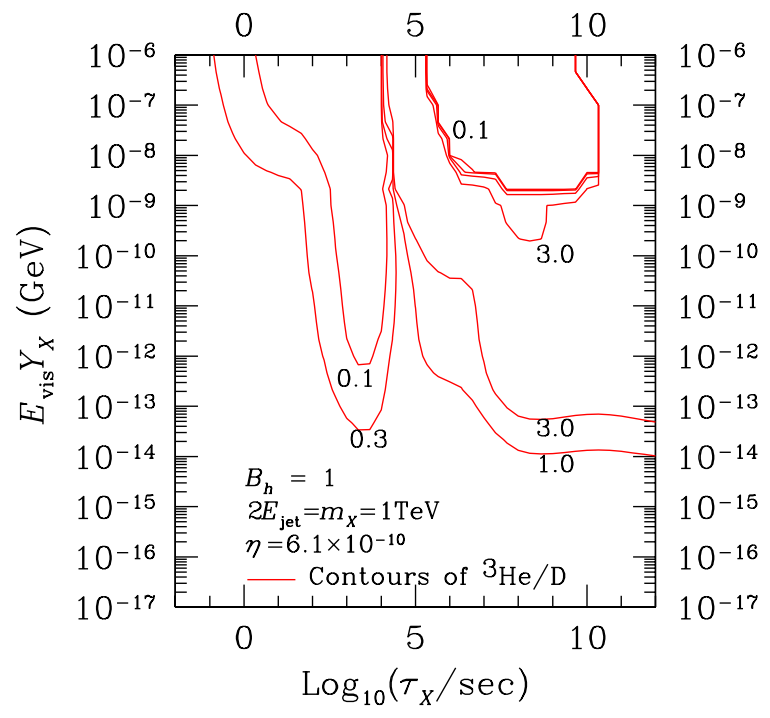

Figure 33: Contours of constant ${ }^{3} \mathrm{He} / \mathrm{D}$. Cosmological and model parameters are the same as Fig. 32, In the $\mathrm{SBBN}$, the theoreical predication is $\left({ }^{3} \mathrm{He} / \mathrm{D}\right)_{\mathrm{SBBN}}=0.335$. 


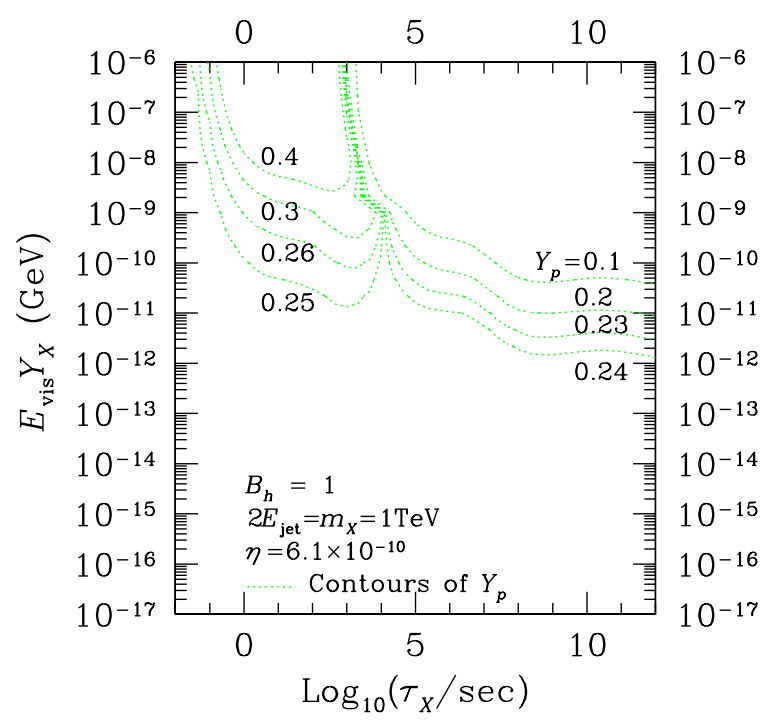

Figure 34: Contours of constant $Y_{p}$. Cosmological and model parameters are the same as Fig. 32. In the SBBN, the theoreical predication is $\left(Y_{p}\right)_{\mathrm{SBBN}}=0.249$.

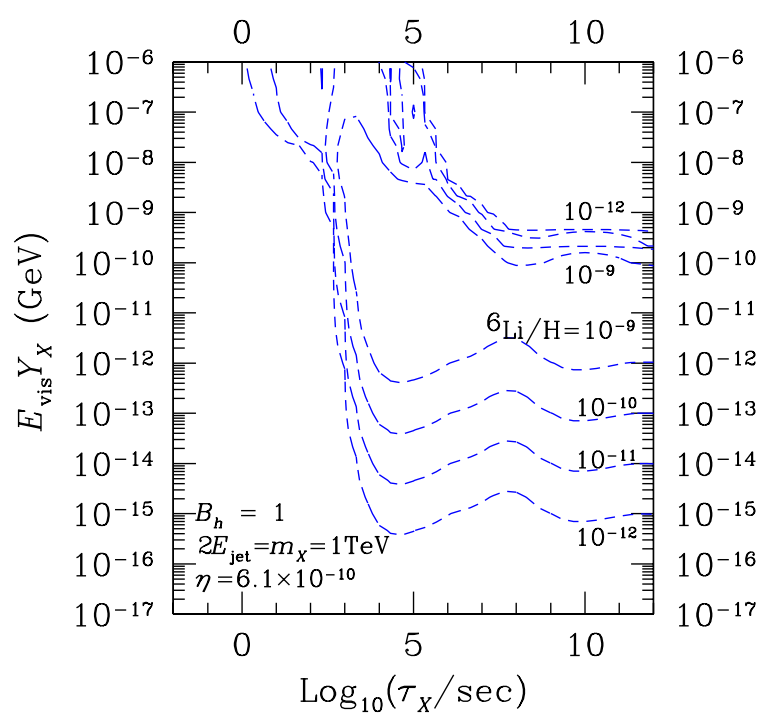

Figure 35: Contours of constant ${ }^{6} \mathrm{Li} / \mathrm{H}$. Cosmological and model parameters are the same as Fig. 32. In the $\mathrm{SBBN}$, the theoreical predication is $\left({ }^{6} \mathrm{Li} / \mathrm{H}\right)_{\mathrm{SBBN}}=1.30 \times 10^{-14}$. 


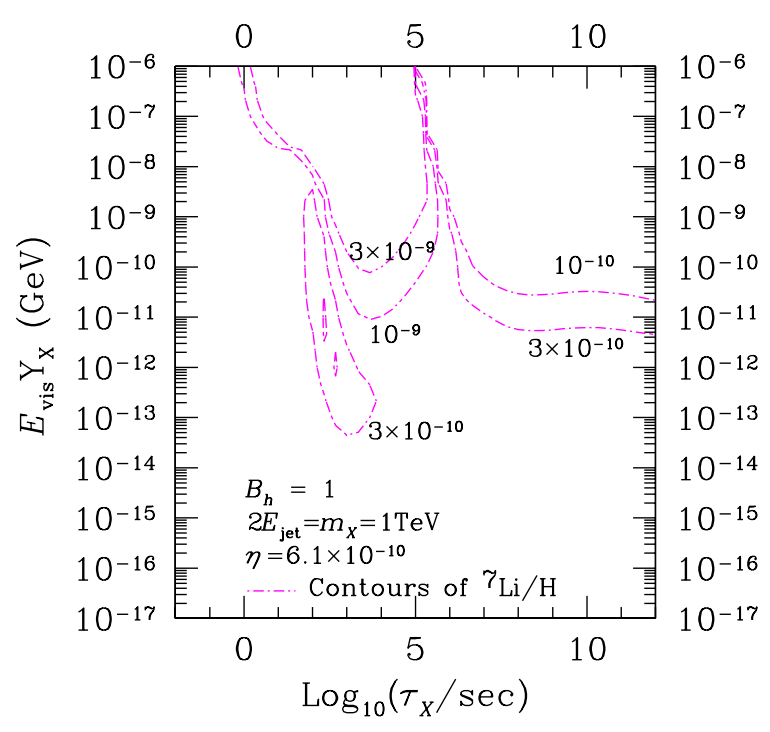

Figure 36: Contours of constant ${ }^{7} \mathrm{Li} / \mathrm{H}$. Cosmological and model parameters are the same as Fig. 32. In the SBBN, the theoreical predication of the abundance is $\left({ }^{7} \mathrm{Li} / \mathrm{H}\right)_{\mathrm{SBBN}}=$ $3.81 \times 10^{-10}$.

\subsection{Predicted light-element abundances}

To see how the abundances of the light elements behave, in Figs. 32, 33, 34, 35] and 36, we plot contours of $\mathrm{D} / \mathrm{H},{ }^{3} \mathrm{He} / \mathrm{D}, Y_{\mathrm{p}},{ }^{6} \mathrm{Li} / \mathrm{H}$, and ${ }^{7} \mathrm{Li} / \mathrm{H}$, in $\left(\tau_{X}, m_{X} Y_{X}\right)$ plane. Here, we used the center values of the cross sections and model parameters; their uncertainties are neglected. In addition, we take $m_{X}=1 \mathrm{TeV}$ and $B_{h}=1$, and consider the case where $X$ decays into two hadronic jets with with the energy $2 E_{\text {jet }}=m_{X}$.

As one can see, for $\tau_{X} \gtrsim 10^{3}-10^{4}$ sec, ${ }^{4} \mathrm{He}$ abundance decreases as the primordial abundance of $X$ becomes larger. This is because, as $Y_{X}$ increases, hadrodissociation and photodissociation processes of ${ }^{4} \mathrm{He}$ become more effective and hence larger number of ${ }^{4} \mathrm{He}$ is destroyed. In addition, since the destruction processes of ${ }^{4} \mathrm{He}$ are followed by the creation processes of $\mathrm{D},{ }^{3} \mathrm{He}$, and ${ }^{6} \mathrm{Li}$, abundances of these light elements first enhanced as $Y_{X}$ increases. If $Y_{X}$ is extremely large, however, all the light elements are destroyed; in this case, abundances of $\mathrm{D},{ }^{3} \mathrm{He}$, and ${ }^{6} \mathrm{Li}$ are also decreased. Contrary to $\mathrm{D},{ }^{3} \mathrm{He}$ and ${ }^{6} \mathrm{Li},{ }^{7} \mathrm{Li}$ is hardly produced. Thus, for larger value of $Y_{X}$, more ${ }^{7} \mathrm{Li}$ is destructed and hence the abundance of ${ }^{7} \mathrm{Li}$ decreases as the primordial abundance of $X$ increases.

On the other hand, for shorter lifetime $\left(\tau_{X} \lesssim 10^{3} \mathrm{sec}\right)$, inter-conversion between the proton and the neutron becomes more effective. In this case, $Y$ and $\mathrm{D} / \mathrm{H}$ increase as $Y_{X}$ increases.

In Fig. 36. we can see a distinctive trend of decrease of ${ }^{7} \mathrm{Li} / \mathrm{H}$ at $\tau_{X} \sim 10^{3}$ sec and $E_{\text {vis }} Y_{X} \gtrsim 10^{-13} \mathrm{GeV}$. That is because free neutrons produced by hadronic showers are 


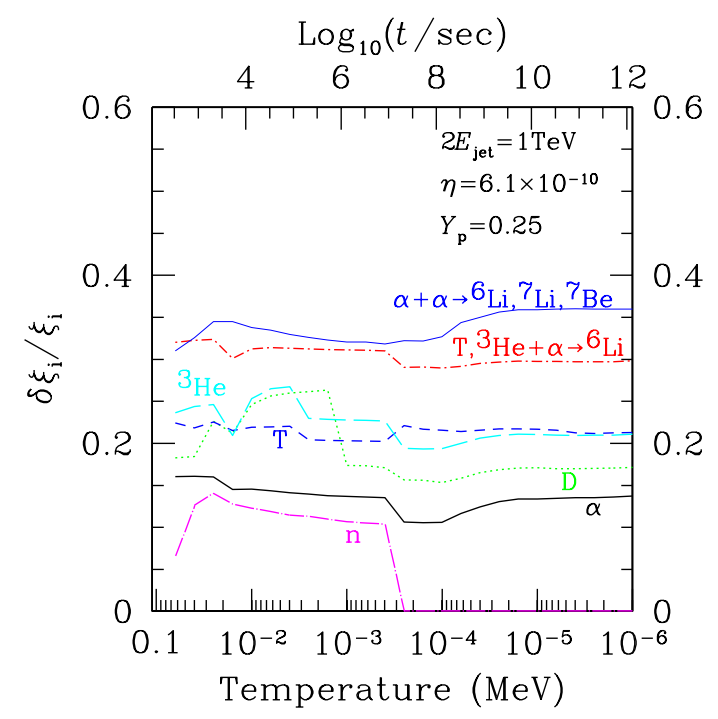

Figure 37: Estimated theoretical errors of the $\xi$-parameters for $m_{X}=1 \mathrm{TeV}, Y_{p}=0.25$ and $\eta=6.1 \times 10^{-10}$.

captured by ${ }^{7} \mathrm{Be}$ through ${ }^{7} \mathrm{Be}\left(n,{ }^{3} \mathrm{He}\right){ }^{4} \mathrm{He}$, which reduces the resultant abundance of ${ }^{7} \mathrm{Li}$. This phenomenon was also pointed in [76].

\subsection{Constraints}

Now we show the constraints on the primordial abundances of $X$, taking into account the theoretical and observational errors. For the execution of Monte Carlo simulation in BBN computation, we should understand the error of all of the reaction rates concerning both radiative and hadronic decay processes. For radiative decay processes, we have shown them in Table 1 .

As for the reaction rates related with the hadrodissociation processes, which arediscussed in Section 7 and Section [8] we should estimate their errors in advance of executing the Monte Carlo simulation. In this work, we assume that the cross sections and the other model parameters of transfered energies, which are used for computing $\xi$ 's, obey the Gaussian distribution with their $1 \sigma$ errors. Computing $\xi$ 's with such errors sufficiently many times (in our caes, one thousand times), we statistically evaluate the errors of $\xi$ 's. Here we adopt $20 \%$ error for all the hadronic cross sections $(\delta \sigma / \sigma=0.2)$, which is larger than typical errors of their experimental data. For the errors of the transfered energies to nuclei in final states after the collisions (the inverse slope parameter $K_{T}$ in inelastic nucleon- $\alpha$ scattering, and slope parameters $B_{\text {sl }}$ in elastic nucleon-nucleon and nucleon- $\alpha$ scatterings (see, Appendix C), we adopt $20 \%$ errors. In addition, as we mentioned in Appendix $\mathrm{C}$, the $\xi$ parameters do not change much with the variations of the inelasticities 
$\left(\kappa_{p}\right.$ and $\left.\kappa_{\alpha}\right)$. Thus, we neglect their uncertainties. Furthermore, we have checked that $\xi$ 's are insensitive to the variation of $\eta$. Thus, we also neglect its uncertainty in evaluating the errors of $\xi$ parameters.

In Fig. 37 we plot the errors of $\xi$ 's as functions of the temperature. These are the case of $m_{X}=1 \mathrm{TeV}, Y_{\mathrm{p}}=0.25$ and $\eta=6.1 \times 10^{-10}$. We have checked that the errors do not change much even if we change the values of $m_{X}$ and $Y_{\mathrm{p}}$. Based on this result, we use the following errors in our Monte Carlo analysis: $\delta \xi_{n} / \xi_{n}=0.15, \delta \xi_{\mathrm{D}} / \xi_{\mathrm{D}}=0.2, \delta \xi_{\mathrm{T}} / \xi_{\mathrm{T}}=0.2$, $\delta \xi^{3} \mathrm{He} / \xi^{3} \mathrm{He}=0.2, \delta \xi_{\alpha} / \xi_{\alpha}=0.2$, and $\delta \xi_{6 \mathrm{Li}}^{\left(\mathrm{T},{ }^{3} \mathrm{He}\right)} / \xi_{6}^{\left(\mathrm{T},{ }^{,} \mathrm{He}\right)}=0.3$.

For the hadron-nucleon inter-conversion reaction rate which was discussed in Section 6 . we adopt $50 \%$ error for each cross section because there are not any adequate experimental data for the uncertainties of cross sections. Therefore, we take the larger errors to get a conservative bound here, for the details, see Ref. [15.

To study the abundance of the light elements with $X$, we have included the effects of the photodissociation, inter-conversion, and the hadrodissociation processes into the BBN calculation. In order to estimate the theoretical uncertainties, we performed the Monte Carlo simulation. Here, we follow the basic procedure explained in [12. In addition, for the BBN calculation, we take account of the observational error of $\eta$ reported by the WMAP collaborations [21] (see Eq. (2.15)).

In our statistical analysis, with a given set of the model parameters, we calculate the theoretical values of the light-element abundances and calculate $\chi_{i}^{2}$, the likelihood variable for individual statistical variable $x_{i}$. For $x_{i}=\left(n_{\mathrm{D}} / n_{\mathrm{H}}\right)$, and $Y$, we use both the upper and lower bounds from the observation, and hence

$$
\chi_{i}^{2}=\frac{\left(\bar{x}_{i}^{\mathrm{th}}-\bar{x}_{i}^{\mathrm{obs}}\right)^{2}}{\left(\sigma_{i}^{\mathrm{th}}\right)^{2}+\left(\sigma_{i}^{\mathrm{obs}}\right)^{2}} \text { for } x_{i}=\left(n_{\mathrm{D}} / n_{\mathrm{H}}\right) \text { and } Y,
$$

where $\bar{x}_{i}^{\text {th }}$ and $\bar{x}_{i}^{\text {obs }}$ are the center values of $x_{i}$ determined from the theoretical calculation and observations, while $\sigma_{i}^{\text {th }}$ and $\sigma_{i}^{\text {obs }}$ are their errors, respectively. In our analysis, $\left(\sigma_{i}^{\text {th }}\right)^{2}$ is calculated by the Monte Carlo analysis. Notice that the $\chi^{2}$ depends on the model parameters through $x_{i}^{\text {th }}$ and $\sigma_{i}^{\text {th }}$. For $x_{i}=r_{3,2}\left(n_{6} \mathrm{Li} / n_{\mathrm{H}}\right)$ and $\log _{10}\left[\left(n_{7} \mathrm{Li} / n_{\mathrm{H}}\right)\right]$ we only use the upper bound. In this case case, we define $\chi_{i}^{2}$ as

$$
\chi_{i}^{2}= \begin{cases}\frac{\left(\bar{x}_{i}^{\mathrm{th}}-\bar{x}_{i}^{\mathrm{obs}}\right)^{2}}{\left(\sigma_{i}^{\mathrm{th}}\right)^{2}+\left(\sigma_{i}^{\mathrm{obs}}\right)^{2}} & : \bar{x}_{i}^{\mathrm{th}}<\bar{x}_{i}^{\mathrm{obs}} \text { for } x_{i}=r_{3,2},\left(n_{6} \mathrm{Li} / n_{\mathrm{H}}\right) \text { and } \log _{10}\left[\left(n_{7 \mathrm{Li}} / n_{\mathrm{H}}\right)\right] . \\ 0 & : \text { otherwise }\end{cases}
$$

Notice that, contrary to the case of SBBN, we do not use the lower bound on $\left(n_{7} \mathrm{Li} / n_{\mathrm{H}}\right)$. This is because we do not include the non-thermal ${ }^{7} \mathrm{Li}$ production processes through $\alpha-\alpha$ collisions. All the observational constraints on primordial abundances of the light elements have been summarized in Section 2 .

In Figs. 38, 39] and 40, we plot the results of the $\chi^{2}$ analysis at $95 \%$ C.L. (i.e., $\chi_{i}^{2}=3.84$ for $x_{i}=\left(n_{\mathrm{D}} / n_{\mathrm{H}}\right)$ and $Y ; \chi_{i}^{2}=2.71$ for $x_{i}=r_{3,2},\left(n_{6 \mathrm{Li}} / n_{\mathrm{H}}\right)$ and $\left.\log _{10}\left[\left(n_{7} \mathrm{Li}_{1} / n_{\mathrm{H}}\right)\right]\right)$ on the 


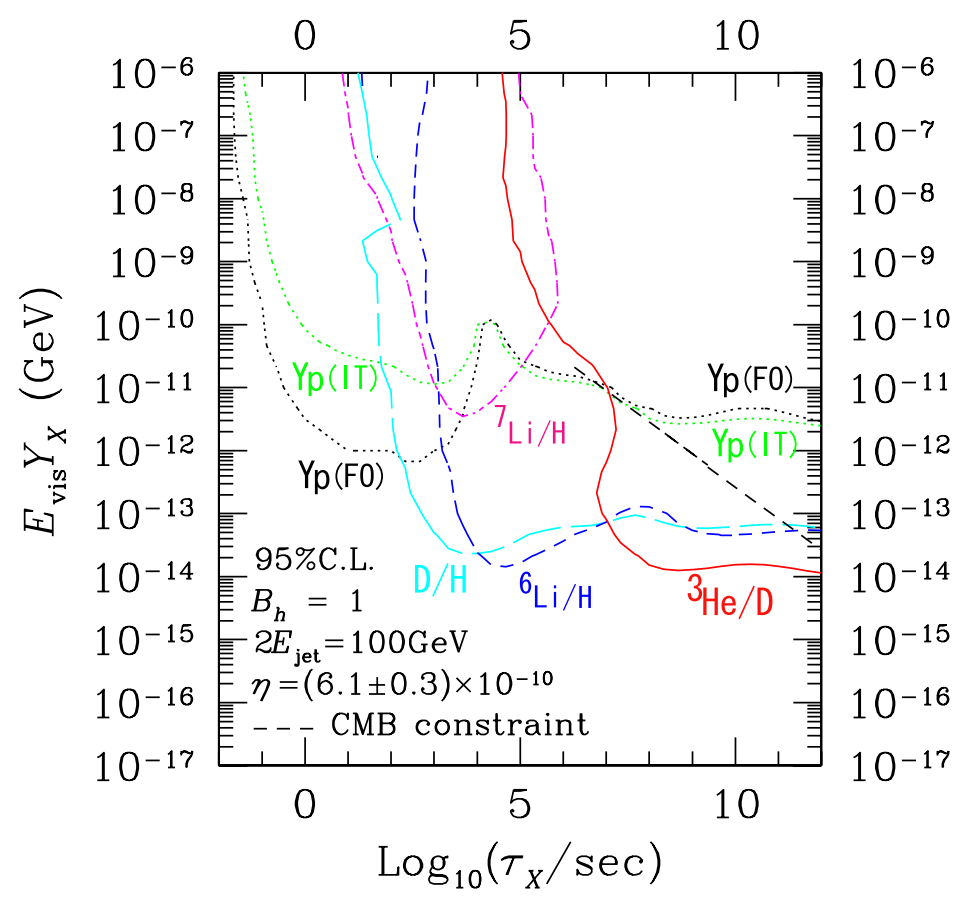

Figure 38: Upper bounds on $m_{X} Y_{X}$ at $95 \%$ C.L. for $B_{h}=1$ and $m_{X}=100 \mathrm{GeV}$. The horizontal axis is the lifetime of $X$. Here we take $m_{X}=100 \mathrm{GeV}$. The straight dashed line is the upper bound by the deviation from the Planck distribution of the CMB.

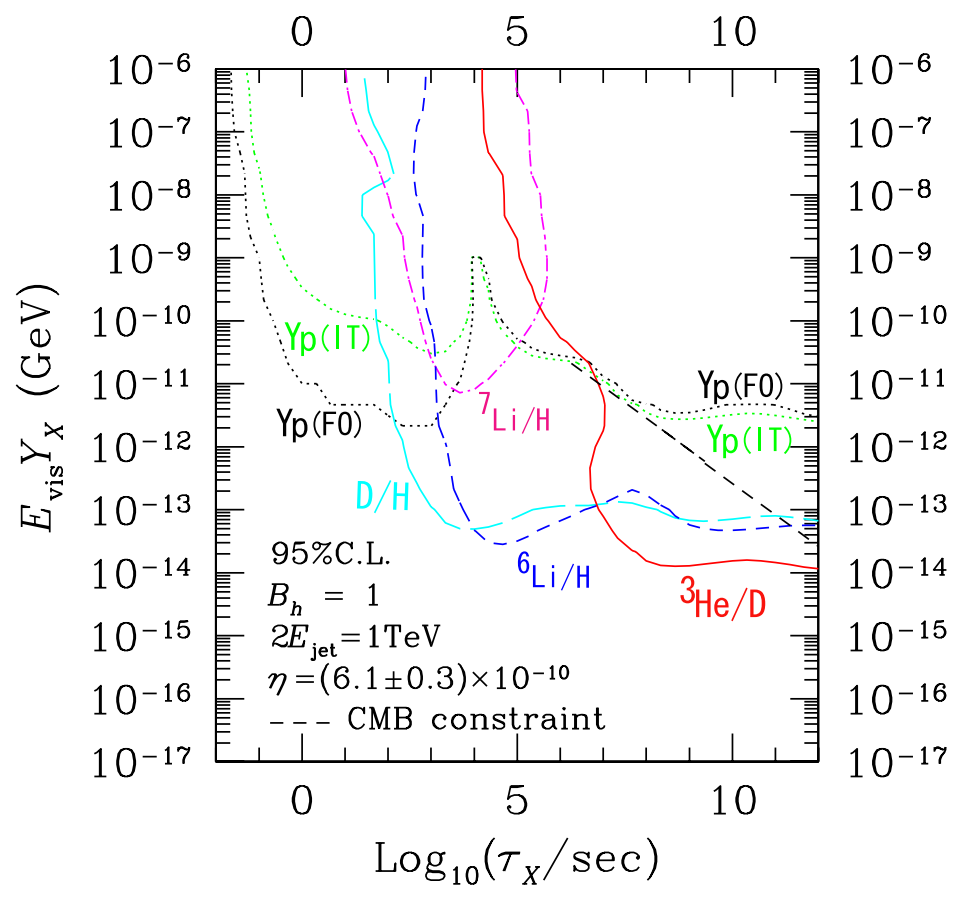

Figure 39: Same as Fig. [38, but for $m_{X}=1 \mathrm{TeV}$. 


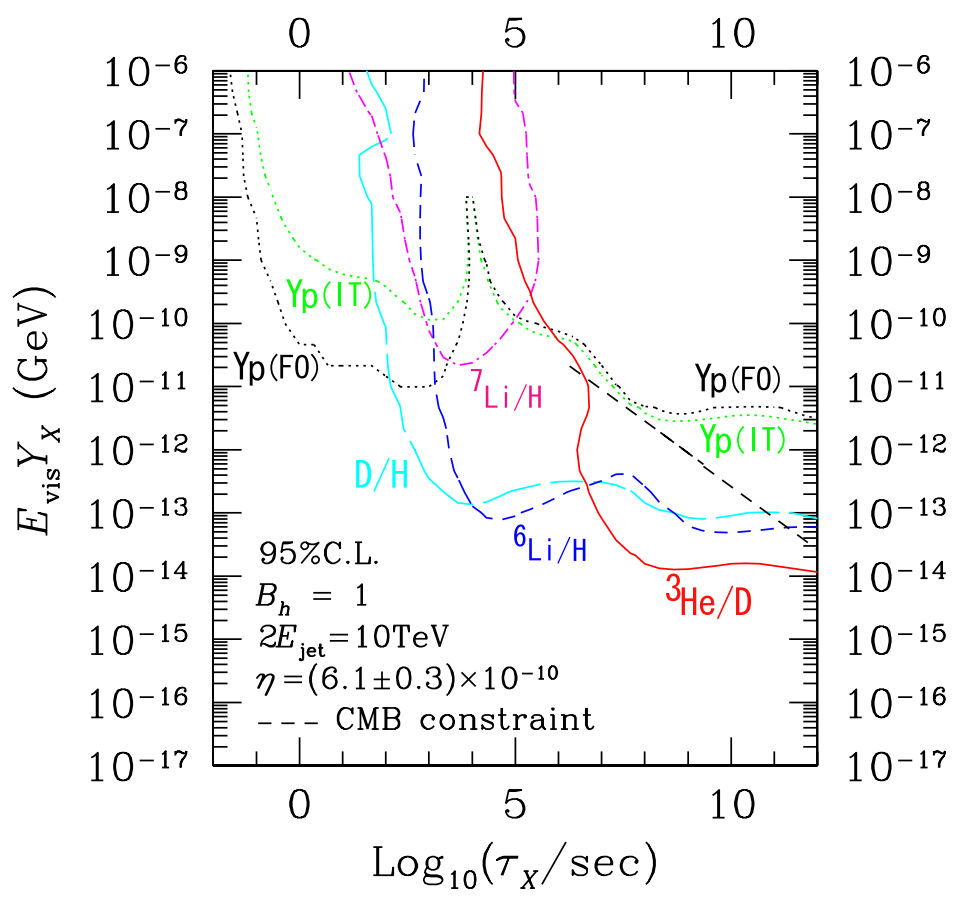

Figure 40: Same as Fig. 38, but for $m_{X}=10 \mathrm{TeV}$.

$\tau_{X}$ vs. $E_{\text {vis }} Y_{X}$ plane for $m_{X}=100 \mathrm{GeV}, 1 \mathrm{TeV}$, and $10 \mathrm{TeV}$, respectively. Here, the hadronic branching ratio is unity, and $X$ decays into two hadronic jets with the energy $2 E_{\text {jet }}=m_{X}$. We also plot the upper bound not to disturb the Planck distribution of the cosmic microwave background. As one can see, constraints on the combination $E_{\mathrm{vis}} Y_{X}$ is quite insensitive to the mass of $X$.

In order to see the dependence on hadronic branching ratio, we also show the results for $B_{h}=10^{-3}$ and $B_{h}=0$ in Figs. 41 and 42 , respectively. $\# 17$ As one can see, with larger value of the hadronic branching ratio, upper bounds on $E_{\mathrm{vis}} Y_{X}$ become severer. In addition, even with a relatively small value of the hadronic branching ratio (i.e., $B_{h}=10^{-3}$ ), the hadronic decay mode may significantly affect the light-element abundances.

With the hadronic decay modes, the most significant constraint on $E_{\mathrm{vis}} Y_{X}$ depends on the lifetime $\tau_{X}$ :

- For $10^{-2}$ sec $\lesssim \tau_{X} \lesssim 10^{2}$ sec, the inter-conversion processes are efficient. In this case, significant amount of $p$ may be converted to $n$ and, consequently, $Y$ may be enhanced. In this case, the constraint from the overproduction of ${ }^{4} \mathrm{He}$ is the most significant.

\footnotetext{
\#17 Our constraint for the case of $B_{h}=0$ is in a reasonable agreement with the results obtained by previous studies (in particular, by Cyburt et al. [16]). Cyburt et al. did not consider the constraint on $n_{3} \mathrm{He} / n_{\mathrm{D}}$ and, for $n_{\mathrm{D}} / n_{\mathrm{H}}$, observational constraint milder than ours is used $\left(1.3 \times 10^{-5}<\left(n_{\mathrm{D}} / n_{\mathrm{H}}\right)^{\text {obs }}<5.3 \times 10^{-5}\right)$. If we adopt $\left(n_{\mathrm{D}} / n_{\mathrm{H}}\right)^{\text {obs }}$ used in [16, the difference between the upper bound on $Y_{X}$ from our analysis and theirs is within the factor of $\sim 3$ or so and is quite mild. It may be due to the difference of the photon spectrum used in the analysis. (For the comparison, notice that we have normalized the yield valiable $Y_{X}$ by the entropy density $s$, and also that $E_{\text {vis }} \simeq \frac{1}{2} m_{X}$ is assumed in [16].)
} 


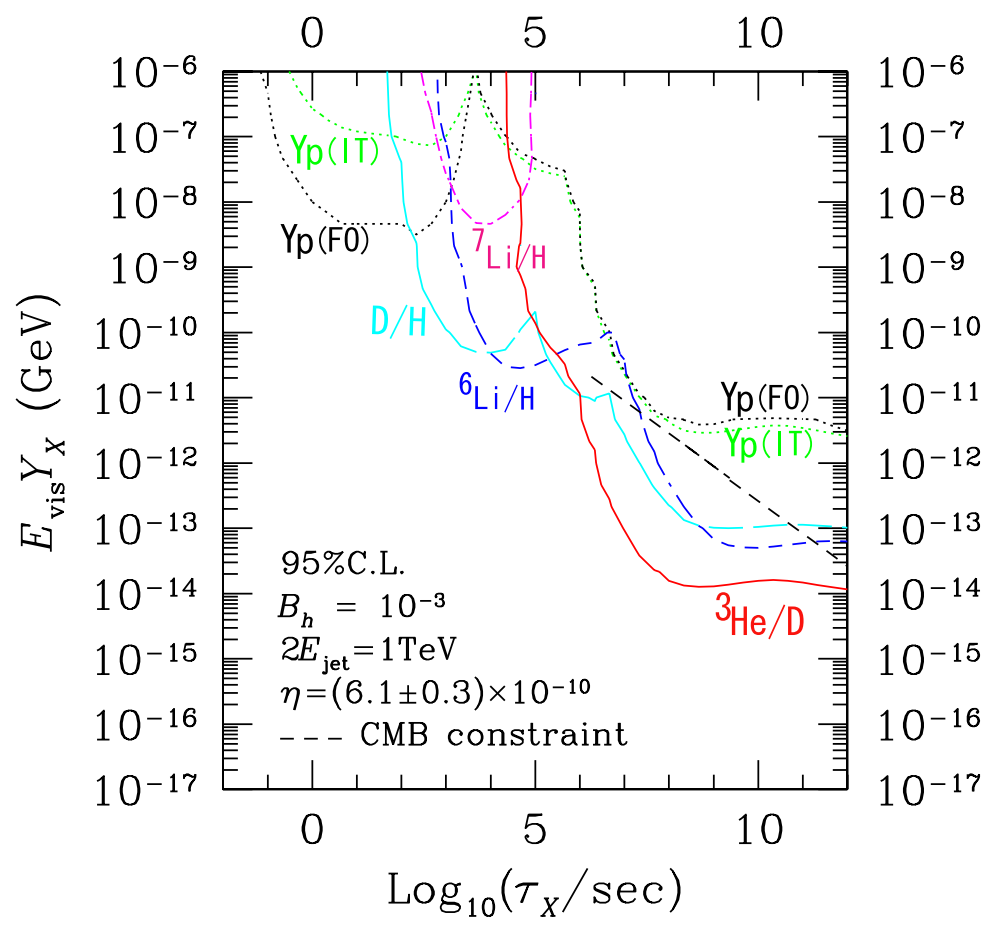

Figure 41: Same as Fig. [38, but for $m_{X}=1 \mathrm{TeV}$ and $B_{h}=10^{-3}$.

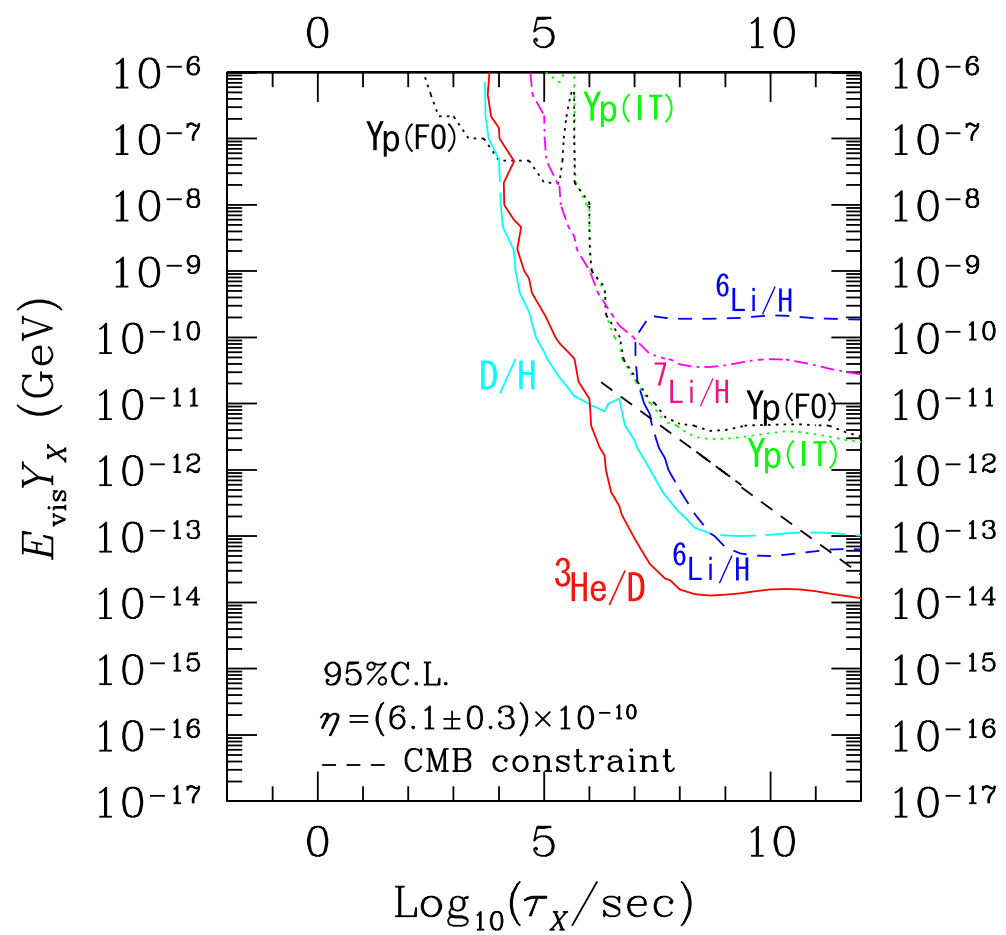

Figure 42: Same as Fig. 38, but for $m_{X}=1 \mathrm{TeV}$ and $B_{h}=0$ (no hadronic decay mode). 
- For $10^{2}$ sec $\lesssim \tau_{X} \lesssim 10^{7}$ sec, energetic hadrons (in particular, neutron) is hardly stopped by the electromagnetic processes and hence the hadrodissociation processes become the most efficient. In particular, in this case, non-thermal productions of D and ${ }^{6} \mathrm{Li}$ provide the most stringent constraint.

- For $10^{7}$ sec $\lesssim \tau_{X} \lesssim 10^{12}$ sec, energetic neutron is likely to decay before scattering off the background nuclei. In this case, effects of the hadronic decay modes become less significant compared to the case with shorter lifetime. Furthermore, in this case, effects of the photodissociation becomes comparable to or more significant than the hadrodissociation. Then, the strongest constraint is from the overproduction of ${ }^{3} \mathrm{He}$.

Importantly, for the case with relatively short lifetime (i.e., $\tau_{X} \lesssim 10^{7} \mathrm{sec}$ ), the hadrodissociation and the inter-conversion processes are the most important. Consequently, the constraints strongly depend on the hadronic branching ratio. In this case, the upper bounds on $E_{\text {vis }} Y_{X}$ are approximately proportional to $B_{\mathrm{h}}$. On the contrary, for longer lifetime (i.e., $\tau_{X} \gtrsim 10^{7} \mathrm{sec}$ ), the most significant constraint is from the overproduction of ${ }^{3} \mathrm{He}$ by the photodissociation of $\alpha_{\mathrm{BG}}$. Then, the upper bound on $E_{\mathrm{vis}} Y_{X}$ becomes insensitive to $B_{\mathrm{h}}$.

Figs. 38 - 42 are our main results and one can read off the constraints on the primordial abundance of long-lived exotic particles. Since our analysis does not assume any special properties of $X$, our results can be applied to various classes of the long-live particles. In the next section, we will discuss one important application of our results, i.e., the application to the gravitino problem.

\section{Application to Unstable Gravitino}

In the previous section, we have derived constraints on the primordial abundance of the late-decaying particle $X$. Now, we apply our results to one of the most important cases, i.e., the case with unstable gravitino. In supergravity theory, the gravitino, which is the superpartner of the graviton, exists. The gravitino acquires a mass $m_{3 / 2}$ from the effect of the supersymmetry breaking. In large class of the models, the gravitino mass is comparable to or larger than $\sim \mathcal{O}(100) \mathrm{GeV}$. Importantly, the interaction of the gravitino is suppressed by inverse powers of the (reduced) Planck scale and hence its interaction is very weak. Thus, if the gravitino is unstable, its lifetime becomes very long. This fact means that, if the gravitino dominantly decays into visible-sector particle and its superpartner, the decay of the gravitino in the early universe may significantly change the predictions of the SBBN unless the primordial abundance of the gravitino is small enough [1].

With the inflation in the early stage of the universe, the primordial gravitino is once diluted but it is produced after the reheating starts. Thus, even in the inflationary models, we may still have the gravitino problem [77]. We can calculate the abundance of the gravitino $Y_{3 / 2}$ as a function of the reheating temperature $T_{R}$, which is defined in this 
paper as

$$
T_{\mathrm{R}} \equiv\left(\frac{10}{g_{*} \pi^{2}} M_{*}^{2} \Gamma_{\text {inf }}^{2}\right)^{1 / 4},
$$

with $\Gamma_{\text {inf }}$ being the decay rate of the inflaton and $g_{*}$ is the effective number of massless degrees of freedom. (Here, we use $g_{*}=228.75$.) In our analysis, we reanalyzed the gravitino production processes in the early universe. In particular, we have used the thermally-averaged gravitino production cross section given in Ref. [78, which properly takes account of the effect of the thermal mass of the gauge bosons, and numerically solved the Boltzmann equation for the gravitino production. (Thus, the following fitting formula is different from the previous ones given in Refs. 9, 78.) The details are discussed in Appendix [F. Then, we find that the gravitino abundance after the inflation is well-fitted by the following formula:

$$
\begin{aligned}
Y_{3 / 2} \simeq & 1.9 \times 10^{-12} \\
& \times\left(\frac{T_{\mathrm{R}}}{10^{10} \mathrm{GeV}}\right)\left[1+0.045 \ln \left(\frac{T_{\mathrm{R}}}{10^{10} \mathrm{GeV}}\right)\right]\left[1-0.028 \ln \left(\frac{T_{\mathrm{R}}}{10^{10} \mathrm{GeV}}\right)\right] .
\end{aligned}
$$

Importantly, the primordial abundance of the gravitino $Y_{3 / 2}$ is approximately proportional to the reheating temperature $T_{\mathrm{R}}$. Consequently, for the inflation models with high reheating temperature, the gravitino abundance may become so large that the light-element abundances are too much affected by the decay of the gravitino. Thus, we obtain a upper bound on the reheating temperature after inflation.

In order to derive the upper bound on $T_{\mathrm{R}}$, we have to specify the decay mode of the gravitino to calculate its lifetime, $B_{h}$, and so on. In this paper, we consider two typical cases. The first case is that the gravitino can directly decay into the pair of colored particles. In particular, we consider the case where the gravitino can decay into gluon and gluino pair: $\psi_{\mu} \rightarrow g+\tilde{g}$ (see Fig. 41). Assuming that this is the dominant decay mode, the lifetime of the gravitino is given by

$$
\tau_{3 / 2}\left(\psi_{\mu} \rightarrow g+\tilde{g}\right) \simeq 6 \times 10^{7} \sec \times\left(\frac{m_{3 / 2}}{100 \mathrm{GeV}}\right)^{-3}
$$

where we have neglected other decay modes (in particular, decay into the lightest neutralino and its superpartner). In this case, the hadronic branching ratio is expected to be very large, so we use $B_{h}=1$. In addition, since the gluino is a colored particle, most of its initial energy is expected to be converted to that of radiation. Thus, even though it is expected that some fraction of the initial energy of gluino is carried away by the lightest neutralino (which is assumed to be the LSP), we use $E_{\text {vis }}=m_{3 / 2}$ in this case.

Direct decay of the gravitino into the colored superparticles may be, however, kinematically blocked. Thus, we also consider the case where the gravitino dominantly decays 
into the photon and the lightest neutralino (which we call "photino" in this section): $\psi_{\mu} \rightarrow \gamma+\tilde{\gamma}$. In this case, the lifetime of the gravitino is obtained as

$$
\tau_{3 / 2}\left(\psi_{\mu} \rightarrow \gamma+\tilde{\gamma}\right) \simeq 4 \times 10^{8} \sec \left(\frac{m_{3 / 2}}{100 \mathrm{GeV}}\right)^{-3}
$$

Even if the gravitino dominantly decays into a photon and a photino, the hadronic branching ratio is non-vanishing since the quark-anti-quark pair can be attached at the end of the virtual photon line. (See Fig. 5.) In this case, $B_{h}$ is expected to be of order $\mathcal{O}\left(\alpha_{\mathrm{em}} / 4 \pi\right)$, and hence we adopt $B_{h}=10^{-3}$. In addition, almost half of the initial energy is carried away by the photino in this case, we use the relation $E_{\text {vis }}=\frac{1}{2} m_{3 / 2}$.

In Figs. 43 and 44, we plot the upper bound on the reheating temperature for the cases where the gravitino dominantly decays into gluon-gluino and photon-photino pairs at $95 \%$ C.L., respectively. For comparison, we also calculated the light-element abundances for the case neglecting the effects of the hadronic decay modes (i.e., for $B_{\mathrm{h}}=0$ ), which only takes into account the effects of the photodissociation. Excluded region for such a case is also shown in the figures by the shaded region.

If the hadronic branching ratio is large (i.e, $B_{h} \sim 1$ ), the constraint on the reheating temperature is very stringent. For example, in the gravity-mediated supersymmetry breaking scenario, the gravitino mass becomes comparable to the masses of the superpartners of the standard-model particles, and hence $m_{3 / 2} \sim \mathcal{O}(100) \mathrm{GeV}$ is expected. In this case, the reheating temperature should be lower than $10^{5}-10^{7} \mathrm{GeV}$. In the anomaly-mediated supersymmetry breaking scenario [79], however, the gravitino mass can be two to three orders of magnitude larger than the masses of the squarks and sleptons. In this case, the upper bound is slightly relaxed, and we obtain $T_{\mathrm{R}} \lesssim 10^{7}-10^{10} \mathrm{GeV}$.

For the case where the gravitino dominantly decays into the photon-photino pair, the upper bound becomes higher. However, the constraint is still much more stringent than the case with $B_{h}=0$. For $m_{3 / 2} \sim \mathcal{O}(100) \mathrm{GeV}$, the upper bound is given by $10^{6}-10^{8} \mathrm{GeV}$. With larger gravitino mass, the constraint becomes less stringent. Since we are assuming $B_{h}=10^{-3}$ here, however, colored superparticles should be heavier than $m_{3 / 2}$. Such a mass spectrum looks quite unnatural from the naturalness point of view.

Note that the above upper bounds on the reheating temperature can be alleviated if the primordial gravitinos are diluted by the decay of particles (such as the thermal inflaton or the moduli fields). Although again gravitino may be also produced directly by the decay or after the reheating via the scattering processes, there are a set of model parameters to make the bounds milder and resolve the gravitino problem. (For the recent study, see, for e.g., Ref. [80]).

The upper bound on the reheating temperature provides significant information about the evolution of the universe, in particular, for the successful scenario of baryogenesis. Since the primary baryon asymmetry is diluted by the inflation, baryogenesis should occur at the temperature lower than $T_{\mathrm{R}}$. For some scenario of baryogenesis, this becomes a very stringent constraint. In particular, for the Fukugita-Yanagida scenario for baryogenesis [81] where the lepton asymmetry generated by the decay of the right-handed neutrino is 


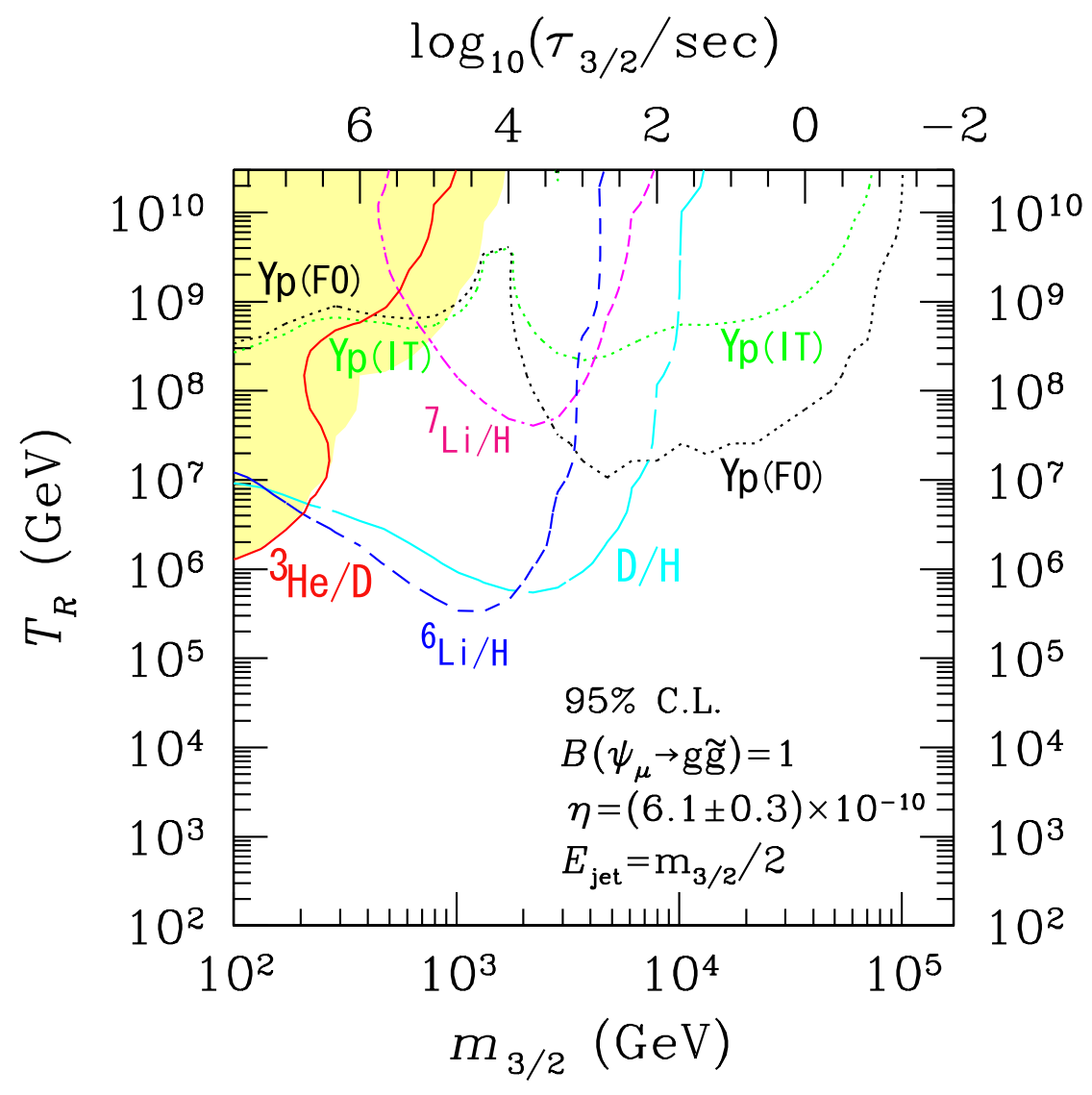

Figure 43: Upper bounds on the reheating temperature as a function of the gravitino mass for the case where the gravitino dominantly decays into gluon-gluino pair. Here, we take $B_{h}=1, E_{\text {vis }}=m_{3 / 2}$, and $E_{\text {jet }}=\frac{1}{2} m_{3 / 2}$. The shaded region is the excluded region for the case with $B_{\mathrm{h}}=0$.

converted to the baryon asymmetry via the spharelon process, mass of the right-handed neutrino should be heavier than $10^{9}-10^{10} \mathrm{GeV} 82$. Thus, for successful scenario of leptogenesis, the reheating temperature is required to be higher than $10^{9}-10^{10} \mathrm{GeV}$. Based on our analysis of the BBN, however, such high reheating temperature is allowed in a very limited case, if the gravitino is unstable. Thus, in order to realize the FukugitaYanagida scenario, we may have to make some assumption on the property of the gravitino, like a very heavy gravitino, a light stable gravitino, or an invisible decay of the gravitino (like decay into the axion-axino pair).

\section{Conclusions and Discussion}

In this paper, we have studied the BBN scenario with exotic late-decaying particle $X$. If there exist an exotic particle with the lifetime longer than $\sim 0.1 \mathrm{sec}$, it decays after 


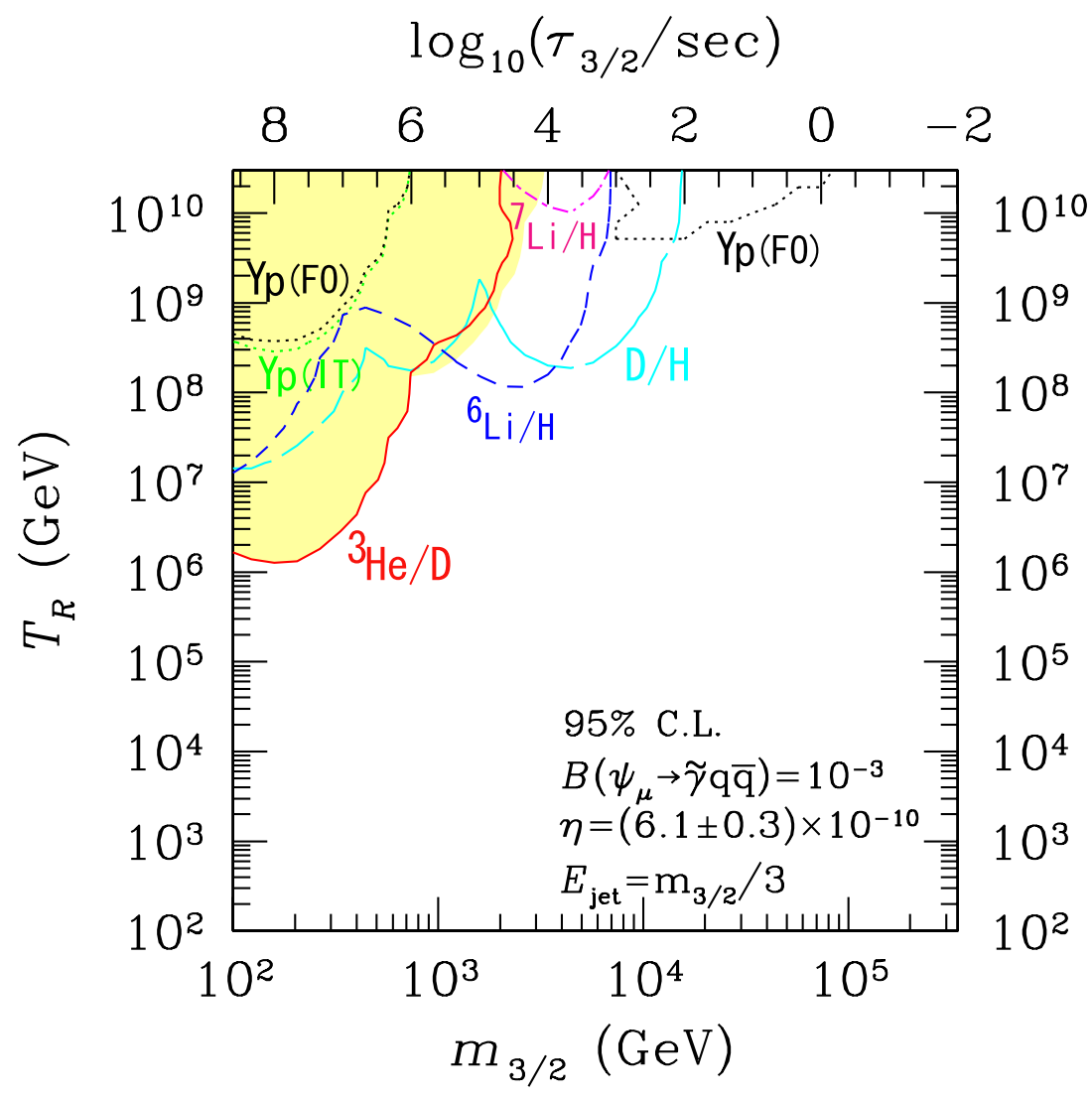

Figure 44: Upper bounds on the reheating temperature as a function of the gravitino mass for the case where the gravitino dominantly decays into photon-photino pair. Here, we take $B_{h}=10^{-3}, E_{\text {vis }}=\frac{1}{2} m_{3 / 2}$, and $E_{\text {jet }}=\frac{1}{3} m_{3 / 2}$. The shaded region is the excluded region for the case with $B_{\mathrm{h}}=0$.

the BBN starts. Decay products of $X$ may cause various non-standard processes, like the photodissociation and hadrodissociation of the background nuclei, and the inter-conversion between the proton and neutron. As a result, abundances of the light elements are affected by the decay of $X$. Since the theoretical predictions of the SBBN scenario is in a reasonable agreements with the observation, decay of such long-lived particle during/after the BBN may spoil the success of the SBBN scenario.

We have derived upper bound on the primordial abundance $X$, paying particular attention to the hadronic decay mode of $X$; as we have seen, as the hadronic branching ratio $B_{\mathrm{h}}$ becomes larger, upper bound on the primordial abundance of $X$ becomes smaller. In particular, for the case where the lifetime of $X$ is $10^{-2}$ sec $\lesssim \tau_{X} \lesssim 10^{2}$ sec, inter-conversion induced by the emitted mesons and nucleons provides the most stringent constraint. For longer lifetime $\left(10^{2} \mathrm{sec} \lesssim \tau_{X} \lesssim 10^{7} \mathrm{sec}\right)$, hadrodissociation of the background $\alpha$ becomes 
effective, resulting in non-thermal productions of various light elements (in particular, D and $\left.{ }^{6} \mathrm{Li}\right)$. If we consider the case with the long enough lifetime $\left(10^{7} \mathrm{sec} \lesssim \tau_{X} \lesssim 10^{12} \mathrm{sec}\right)$, ${ }^{3} \mathrm{He}$ is overproduced by the photodissociation and hadrodissociation of $\alpha_{\mathrm{BG}}$, which gives the most stringent constraint on the primordial abundances of $X$. (See figures in Section 9.)

Since our analysis has been done without specifying the detailed properties of $X$, our results can be applied to various exotic long-lived particles. Some of the examples are the gravitinos, cosmological moduli fields, and the NLSP for the case where the gravitino is the LSP. As an application of our analysis, we considered the unstable gravitino and applied our results to the gravitino problem. In particular, for the cases where the gravitino dominantly decays into gluon-gluino and photon-photino pairs, we derived the upper bound on the reheating temperature after inflation. For the case of the gravity-mediated supersymmetry breaking, gravitino mass is expected to be $\sim \mathcal{O}(100) \mathrm{GeV}$. In this case, even if the hadronic branching ratio is $\sim \mathcal{O}\left(10^{-3}\right)$, the reheating temperature is constrained to be smaller than $10^{6}-10^{8} \mathrm{GeV}$. If the gravitino mass is much larger than $\sim \mathcal{O}(100) \mathrm{GeV}$, the constraint on $T_{\mathrm{R}}$ may be relaxed. With such gravitino, however, the hadronic branching ratio would be close to 1 since, in such a case, all the superpartners of the standard-model particles are expected to be lighter than the gravitino from the naturalness point of view. (Such a mass spectrum may be realized in the anomaly-mediated supersymmetry breaking scenario.) For $m_{3 / 2} \sim \mathcal{O}(10-100) \mathrm{TeV}$ with $B_{\mathrm{h}} \sim 1$, the upper bound is given by $T_{\mathrm{R}} \lesssim 10^{7}-10^{10} \mathrm{GeV}$. (See figures in Section [10])

\section{Acknowledgements}

The authors wish to thank J. Arafune, A. Brandenburg, R.H. Cyburt, T. Hatsuda, J.E. Kim, A. Kohama and K. Maki for valuable discussions and suggestions. K.K. also thanks T. Asaka, O. Biebel, S. Mihara, M.M. Nojiri, H. Sakai, T. Sjöstrand, F. Takahara and J. Yokoyama for useful comments. This work was partially supported by the Grants-in Aid of the Ministry of Education, Science, Sports, and Culture of Japan No. 14540245 (MK), 15-03605 (KK) and 15540247 (TM).

\section{A Photon Spectrum}

In this appendix, we summarize the properties of the photon spectrum generated by the high energy photon from radiative decay of a heavy unstable particle. (Details of the calculation of the photon spectrum is discussed in 9.)

Once the high energy photon is injected into the thermal bath consisting of the photon, (non-relativistic) electron, and nuclei, the high energy photon induces cascade processes. We have calculated the photon spectrum taking account of effects of the following processes: 
- Injection of the high energy photon from the radiative decay of $X$

- Double photon pair creation $\left(\gamma+\gamma_{\mathrm{BG}} \rightarrow e^{+}+e^{-}\right)$

- Photon-photon scattering $\left(\gamma+\gamma_{\mathrm{BG}} \rightarrow \gamma+\gamma\right)$

- Compton scattering off thermal electron $\left(\gamma+e_{\mathrm{BG}}^{-} \rightarrow \gamma+e^{-}\right)$

- Inverse Compton scattering off background photon $\left(e^{ \pm}+\gamma_{\mathrm{BG}} \rightarrow e^{ \pm}+\gamma\right)$

- Pair creation in background proton (and $\left.\alpha_{\mathrm{BG}}\right)\left(\gamma+p_{\mathrm{BG}} \rightarrow e^{+}+e^{-}+p\right)$

Here, the subscript "BG" indicates that the corresponding particles are in the thermal bath. In addition, in the process $\gamma+p_{\mathrm{BG}} \rightarrow e^{+}+e^{-}+p$, the background proton plays the role of the source of the electric field.

The photon spectrum is determined by following the distribution functions of the photon and electron, $f_{\gamma}$ and $f_{e}$. Since the expansion rate of the universe is much smaller than the scattering rates of the electromagnetic processes, the relevant Boltzmann equations to be solved are written in the following forms

$$
\begin{aligned}
\frac{\partial f_{\gamma}\left(E_{\gamma}\right)}{\partial t}= & {\left[\frac{\partial f_{\gamma}\left(E_{\gamma}\right)}{\partial t}\right]_{\mathrm{DP}}+\left[\frac{\partial f_{\gamma}\left(E_{\gamma}\right)}{\partial t}\right]_{\mathrm{PP}}+\left[\frac{\partial f_{\gamma}\left(E_{\gamma}\right)}{\partial t}\right]_{\mathrm{PC}}+\left[\frac{\partial f_{\gamma}\left(E_{\gamma}\right)}{\partial t}\right]_{\mathrm{CS}} } \\
& +\left[\frac{\partial f_{\gamma}\left(E_{\gamma}\right)}{\partial t}\right]_{\mathrm{IC}}+\left[\frac{\partial f_{\gamma}\left(E_{\gamma}\right)}{\partial t}\right]_{\mathrm{DE}}, \\
\frac{\partial f_{e}\left(E_{e}\right)}{\partial t}= & {\left[\frac{\partial f_{e}\left(E_{e}\right)}{\partial t}\right]_{\mathrm{DP}}+\left[\frac{\partial f_{e}\left(E_{e}\right)}{\partial t}\right]_{\mathrm{PC}}+\left[\frac{\partial f_{e}\left(E_{e}\right)}{\partial t}\right]_{\mathrm{CS}}+\left[\frac{\partial f_{e}\left(E_{e}\right)}{\partial t}\right]_{\mathrm{IC}} } \\
& +\left[\frac{\partial f_{e}\left(E_{e}\right)}{\partial t}\right]_{\mathrm{DE}}
\end{aligned}
$$

where the terms with the subscripts "DP," "PP," "PC," "CS," "IC" and "DE" denote the contributions of double-photon pair creation, photon-photon scattering, pair creation in nuclei, Compton scattering, inverse Compton scattering, and the radiative decay of $X$, respectively.

Importantly, for the energy region $E_{\gamma} \ll E_{\gamma, 0}$ (with $E_{\gamma, 0}$ being the energy of the injected photon from the radiative decay of $X$ ), the function $f_{\gamma}\left(E_{\gamma}\right)$ is determined by the total amount of injected energy per unit time; with this quantity being fixed, $f_{\gamma}\left(E_{\gamma}\right)$ is insensitive to $E_{\gamma, 0}$. Thus, in the BBN with the radiatively decaying particle $X$, resultant abundances of the light elements primarily depend on the lifetime of $X$ and the combination $E_{\gamma, 0} Y_{X}$, but are insensitive to $E_{\gamma, 0}$ if the combination $E_{\gamma, 0} Y_{X}$ is fixed. (For the case with the hadronic decay processes, they also depend on the hadronic branching ratio.)

In Fig. 45, we plot the photon spectrum $f_{\gamma}\left(E_{\gamma}\right)$ for several values of the background temperature $T$. As we mentioned, first of all, $f_{\gamma}\left(E_{\gamma}\right)$ is insensitive to the energy of the injected photon as far as the total amount of the injected energy per unit time is fixed. In 


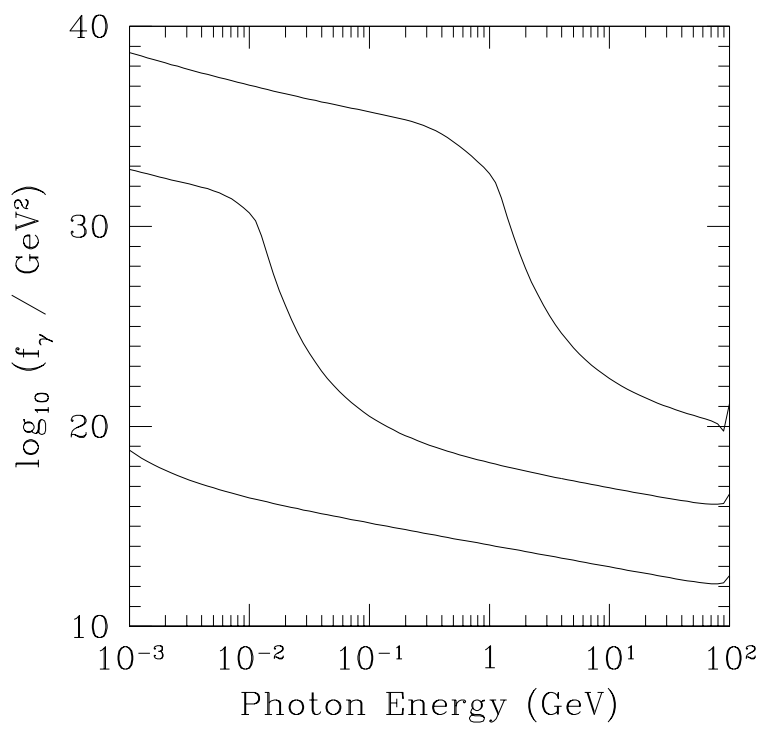

Figure 45: The photon spectrum for the background temperature $T=10 \mathrm{eV}, 1 \mathrm{keV}$, and $100 \mathrm{keV}$ (from above). Here, $E_{\gamma, 0}=100 \mathrm{GeV}$ and the flux of the injected photon is $\left[\partial f_{\gamma} / \partial t\right]_{\mathrm{DE}}=\delta\left(E_{\gamma}-E_{\gamma, 0}\right) \mathrm{GeV}^{-4}$ while $\left[\partial f_{e} / \partial t\right]_{\mathrm{DE}}=0$.

addition, $f_{\gamma}\left(E_{\gamma}\right)$ drastically changes its value at $E_{\gamma} \sim m_{e}^{2} / 22 T$. This can be understood as follows. Photons with high enough energy easily scatter off the background photon and create $e^{+} e^{-}$pairs. Since the number density of the background photon is much larger than those of the electron and nuclei, the pair creation process is extremely efficient for photons with $E_{\gamma} \gtrsim m_{e}^{2} / 22 T$ the number density of the photons with such energy is suppressed. As $E_{\gamma}$ becomes lower, however, the process $\gamma+\gamma \rightarrow e^{+}+e^{-}$is kinematically blocked, and hence $f_{\gamma}\left(E_{\gamma}\right)$ is no longer suppressed.

\section{B Energy Loss Rates}

In this Appendix, we summarize the energy-loss rates of high-energy hadrons in the electromagnetic plasma. Here, we consider the energy-loss of the hadron with mass $m_{H}=A m_{N}$ and charge $Z$. In addition, $E_{\text {kin }}, \gamma$, and $\beta$ are used for the kinetic energy, Lorentz factor, and velocity of the hadron, respectively.

In discussing the energy-loss processes via the electromagnetic interactions, one of the most important processes is the scattering with background electron (and positron). In calculating the scattering rate, it is necessary to determine the number density of the background electron (and positron). In our analysis, for the sum of the number density of the electron and positron in the background (called $n_{e}$ ), we use the following approximated 
formula which well agrees with the exact formula:

$$
n_{e}=n_{e^{-}}+n_{e^{+}}= \begin{cases}\frac{3}{2} n_{\gamma} & : T \geq m_{e} \\ 4\left(\frac{m_{e} T}{2 \pi}\right)^{3 / 2} e^{-m_{e} / T} & : \quad m_{e}>T \geq m_{e} / 26 . \\ \left(1-\frac{1}{2} Y\right) \eta n_{\gamma} & : \quad T<m_{e} / 26\end{cases}
$$

\section{B.1 Energy loss of charged particles}

First, we consider the energy-loss rates of charged particles (i.e, $p, \mathrm{D}, \mathrm{T},{ }^{3} \mathrm{He},{ }^{4} \mathrm{He}$, and $\left.\pi^{ \pm}\right)$. For those charged particles, the Coulomb, Compton, and Bethe-Heitler scattering are important.

First of all, we summarize the energy-loss rate through the Coulomb scattering. (For details, see Ref. [7].) For the temperature higher than the electron mass $\left(T \gtrsim m_{e}\right)$, the energy-loss rate of relativistic charged particle is given by

$$
\left[\frac{d E_{(\mathrm{ch})}}{d t}\right]_{\text {Coulomb }}=-\frac{\pi}{3} Z^{2} \alpha_{\mathrm{em}}^{2} T^{2} \Lambda: T \gtrsim m_{e}, E_{\mathrm{kin}} \gtrsim m_{H},
$$

where $\Lambda=\ln \left[2 /\left(1-\cos \theta_{\min }\right)\right]-1$ with $\theta_{\min }$ being the minimal scattering angle in the center-of-mass frame. In discussing the energy-loss process in the thermal plasma, $\theta_{\min }$ is determined by the requirement that the energy transfer to the electron in the comoving frame be smaller than the plasma frequency $\omega_{\mathrm{p}}$ [97] which is given by

$$
\omega_{\mathrm{p}}^{2}=\frac{4 \pi \alpha_{\mathrm{em}} n_{e}}{m_{e}} .
$$

Thus, in our analysis, we use the approximated formula

$$
\Lambda \simeq \ln \left(\frac{\tilde{\gamma}^{2} \tilde{\beta}^{2} m_{e}}{\omega_{p}}\right)
$$

where $\tilde{\beta}$ is the required velocity to go to the center-of-mass frame and $\tilde{\gamma}=\left(1-\tilde{\beta}^{2}\right)^{-1 / 2}$. Notice that, when the incident charged particle is non-relativistic in the center of mass frame, $\tilde{\gamma} \tilde{\beta} \simeq \gamma \beta$. For nonrelativistic charged particle, we obtain

$$
\left[\frac{d E_{(\mathrm{ch})}}{d t}\right]_{\text {Coulomb }}=-\frac{4 \pi}{9} \frac{E_{\text {kin }}}{m_{H}} Z^{2} \alpha_{\mathrm{em}}^{2} T^{2} \Lambda: T \gtrsim m_{e}, E_{\text {kin }} \lesssim m_{H} .
$$

For lower temperature $\left(T \lesssim m_{e}\right)$, the formula of the energy-loss rate changes. For relativistic charged particles, we obtain

$$
\left[\frac{d E_{(\mathrm{ch})}}{d t}\right]_{\text {Coulomb }}=-4 \pi Z^{2} \alpha_{\mathrm{em}}^{2} \frac{n_{e}}{m_{e}} \Lambda \quad: \quad T \lesssim m_{e}, E_{\mathrm{kin}} \gtrsim m_{H} .
$$


For non-relativistic particles, we need special care since the energy-loss rate strongly depends on the relative velocity between the charged hadron and the background electron. Denoting the distribution function of the background electron (as a function of its velocity $\left.\beta_{e}\right)$ as $f_{e_{\mathrm{BG}}}\left(\beta_{e}\right)$, the energy-loss rate of the non-relativistic hadrons is given by

$$
\begin{aligned}
{\left[\frac{d E_{(\mathrm{ch})}}{d t}\right]_{\text {Coulomb }}=} & -\frac{4 \pi Z^{2} \alpha_{\mathrm{em}}^{2}}{m_{e} \beta} \Lambda \int_{\beta_{e}<\beta} d^{3} \vec{\beta}_{e} f_{e_{\mathrm{BG}}}\left(\beta_{e}\right) \\
& -\frac{4 \pi Z^{2} \alpha_{\mathrm{em}}^{2} \beta^{2}}{3 m_{e}} \Lambda \int_{\beta_{e}>\beta} d^{3} \vec{\beta}_{e} \beta_{e}^{-1} f_{e_{\mathrm{BG}}}\left(\beta_{e}\right): T \lesssim m_{e}, E_{\mathrm{kin}} \lesssim m_{H} .
\end{aligned}
$$

Notice that, the integral in the first term of the right-hand side of the above equation is the number density of the background electron with velocity $\beta_{e}<\beta$. Since the hadron and the electron are both non-relativistic, contribution of the background electron with $\beta_{e}>\beta$ more suppressed than the contribution of the electrons with $\beta_{e}<\beta$. Then, neglecting the second term of the right-hand side of Eq. (B.7), we obtain

$$
\left[\frac{d E_{(\mathrm{ch})}}{d t}\right]_{\text {Coulomb }} \simeq-\frac{4 \pi \alpha_{\mathrm{em}}^{2} Z^{2} n_{e}}{m_{e} \beta} I\left(\beta / \sqrt{2 T / m_{e}}\right) \Lambda,
$$

where

$$
I(r)=\frac{4}{\sqrt{\pi}} \int_{0}^{r} d x x^{2} e^{-x^{2}}
$$

Another important process is the Compton scattering. In particular, ultra-relativistic charged particles may lose significant amount of energy by scattering off the background photons. For the Compton scattering process, the energy loss rate is approximately given by

$$
\left[\frac{d E_{(\mathrm{ch})}}{d t}\right]_{\text {Compton }}=-\frac{32 \pi^{3}}{135} \alpha_{\mathrm{em}}^{2} \frac{\gamma^{2}-1}{m_{H}^{2}} T^{4} .
$$

We use this formula in our analysis.

Next, we consider the Bethe-Heitler process (e.g., for the proton projectile $p+\gamma \rightarrow$ $p+e^{+}+e^{-}$) 98]. This process is important for relativistic charged nuclei with energy $E \gtrsim A m_{H} m_{e} / \epsilon_{\gamma} \sim 1.6 A \mathrm{GeV} \times(T / 0.1 \mathrm{MeV})^{-1}$, where $A$ is the mass number and $\epsilon_{\gamma}$ is the photon energy. The fitting formula of the energy loss rate is given by 99

$$
\left[\frac{d E_{(\mathrm{ch})}}{d t}\right]_{\mathrm{BH}}=-\alpha_{\mathrm{em}} r_{0}^{2} Z^{2} m_{e}^{4} \int_{2}^{\infty} d \kappa f_{\gamma_{\mathrm{BG}}}(\kappa / 2 \gamma) \frac{\phi(\kappa)}{\kappa^{2}}
$$

where $r_{0}$ is the classical electron radius $\left(=\alpha_{\mathrm{em}} / m_{e}\right)$, and $f_{\gamma_{\mathrm{BG}}}$ is the distribution function of the background photon which is given by

$$
f_{\gamma_{\mathrm{BG}}}(\epsilon)=\left(\frac{\epsilon}{\pi}\right)^{2} \frac{1}{\exp (\epsilon / T)-1} .
$$


In addition, the function $\phi(\kappa)$ in the integrand is fitted by 100

$$
\phi(\kappa)=\left\{\begin{array}{ll}
\frac{\pi}{12} \frac{(\kappa-2)^{4}}{1+\sum_{i=1}^{4} a_{i}(\kappa-2)^{i}} & : \quad \kappa \leq 25 \\
\frac{\kappa \sum_{j=0}^{3} b_{j} \ln ^{j}(\kappa)}{1-\sum_{k=1}^{3} c_{k} / \kappa^{k}} & : \kappa>25
\end{array},\right.
$$

where the coefficients are given by

$$
\begin{aligned}
& a_{1}=0.8048, \quad a_{2}=0.1459, \quad a_{3}=1.137 \times 10^{-3}, \quad a_{4}=-3.879 \times 10^{-6}, \\
& b_{0}=-86.07, \quad b_{1}=50.96, \quad b_{2}=-14.45, \quad b_{3}=\frac{8}{3}
\end{aligned}
$$

and

$$
c_{1}=2.910, \quad c_{2}=78.35, \quad c_{3}=1.837 \times 10^{3} .
$$

Although we include the photo-pion process $(p+\gamma \rightarrow p(n)+\pi)$ into our analysis, it is important only for highly-relativistic nucleons with energy $E \gtrsim m_{N} m_{\pi} / \epsilon_{\gamma} \sim 4.7 \times$ $10^{2} \mathrm{GeV} \times(T / 0.1 \mathrm{MeV})^{-1}$. The fitting formula of the energy loss rate is approximately given by 101 ]

$$
\left[\frac{d E_{(\mathrm{ch})}}{d t}\right]_{\text {photo-pion }}=\left\{\begin{array}{l}
-\frac{2}{\pi^{2}} \sigma^{\prime} \frac{\epsilon_{0}^{2}}{m_{N}} T^{3} E \exp \left(-\frac{\epsilon_{0} m_{N}}{2 E T}\right) \\
-1.8 \times 10^{-8} \mathrm{yr}^{-1} E\left(\frac{T}{2.7 \mathrm{~K}}\right)^{3}: E \geq \epsilon_{0} m_{N} / T \\
\quad: E \epsilon_{0} m_{N} / T
\end{array},\right.
$$

where $\epsilon_{0}=m_{\pi}\left(1+\frac{m_{\pi}}{2 m_{N}}\right)$ is the (approximated) threshold energy of the photon for the photo-pion process in the rest frame of nucleon, and $\sigma^{\prime} \simeq 6.8 \times 10^{-36} \mathrm{~cm}^{2} / \mathrm{eV}$ is a constant.

\section{B.2 Energy loss of neutral particles}

Now, we consider the energy-loss of the neutral particles, in particular, the neutron which is the only relevant neutral particle for which the energy-loss rate should be discussed. For the neutron, scattering with the background electron with magnetic-moment interaction is the most important process for the energy-loss. (Even for the neutron, we call such a process as "Coulomb scattering.") For $T \gtrsim m_{e}$, we obtain

$$
\left[\frac{d E_{n}}{d t}\right]_{\text {Coulomb }}= \begin{cases}-\frac{7 \pi^{3}}{15} \alpha_{\mathrm{em}}^{2} \frac{g_{n}^{2}}{m_{N}^{2}}\left(\frac{E_{\mathrm{kin}}}{m_{N}}\right)^{2} T^{4} & : T \gtrsim m_{e}, E_{\mathrm{kin}} \gtrsim m_{N} \\ -\frac{14 \pi^{3}}{15} \alpha_{\mathrm{em}}^{2} \frac{g_{n}^{2}}{m_{N}^{3}} T^{4} E_{\mathrm{kin}}: T \gtrsim m_{e}, E_{\mathrm{kin}} \lesssim m_{N}\end{cases}
$$


where $g_{n} \simeq-1.913$ is the neutron magnetic moment 66. Furthermore, for $T \lesssim m_{e}$, the energy-loss rate is given by $\# 18$

$$
\left[\frac{d E_{n}}{d t}\right]_{\text {Coulomb }}= \begin{cases}-\frac{3 \pi \alpha_{\mathrm{em}}^{2} g_{n}^{2} m_{e}}{m_{N}^{4}} n_{e} E_{\mathrm{kin}}^{2} & : T \lesssim m_{e}, E_{\mathrm{kin}} \gtrsim m_{N} \\ -\frac{16 \alpha_{\mathrm{em}}^{2} g_{n}^{2}}{3 \pi m_{N}^{3}} T^{4} e^{-x_{e}} G_{e}\left(x_{e}\right) E_{\mathrm{kin}} & : T \lesssim m_{e}, E_{\mathrm{kin}} \lesssim m_{N}\end{cases}
$$

where $G_{e}\left(x_{e}\right)=x_{e}^{3}+3 x_{e}^{2}+6 x_{e}+6$ with $x_{e}=m_{e} / T$.

As well as the case of charged particles, the photo-pion process $(n+\gamma \rightarrow n(p)+\pi)$ is also important for highly-relativistic neutrons, see Eq. (B.17). We include this photo-pion energy loss rate in our analysis. Note also that, because the energy loss rate through $n+\gamma \rightarrow n+\gamma$ is smaller than the Coulomb energy-loss rate [7, 86], we do not include this process in estimating the energy loss rate of the beam neutron.

\section{Energy Transfer}

In this appendix, we discuss the energy transfers into the scattered/produced particles after the elastic and inelastic hadronic scattering processes. There are three types of energetic nuclei after the processes; scattered target nucleus ( $p$ or $\alpha$ ), beam nucleon after the scattering, and daughter nuclei which are produced by the destruction of $\alpha$. In order to study the evolution of the hadronic shower, it is necessary to consider how the energy of the initial-state particles are transferred to these final-state ones.

Throughout this appendix, $p_{i}=\left(E_{i}, \boldsymbol{p}_{i}\right)$ denotes the four-dimensional momentum of $N_{i}$ (or $A_{i}$ ) with its mass $m_{i}$ in the rest frame of the target particle (i.e., comoving frame of the expanding universe), and $K_{i}=E_{i}-m_{i}$ is its kinetic energy. The dashed quantities are those for the final state particles. In addition, the mass difference between the proton and neutron is not important here so we use the approximated relation $m_{p} \simeq m_{n} \equiv m_{N}$. Furthermore, mass of a nucleus $A_{i}$ is also approximated by the relation $m_{A_{i}} \simeq A_{A_{i}} m_{N}$ where $A_{A_{i}}$ is the atomic number of $A_{i}$.

${ }^{\# 18}$ When the temperature become so low (i.e., $\left.T \lesssim m_{e} / 26\right)$ that the number of the electron in the comoving volume becomes constant, expression for the non-relativistic case given in Eq. (B.19) becomes invalid. For such a low temperature, the energy-loss rate of the non-relativistic neutron is given by

$$
\left[\frac{d E_{n}}{d t}\right]_{\text {Coulomb }}=-\frac{4 \alpha_{\mathrm{em}}^{2} g_{n}^{2}}{3 \pi m_{N}^{3}} n_{e}\left(\frac{2 \pi}{m_{e} T}\right)^{3 / 2} m_{e}^{3} T E_{\mathrm{kin}} .
$$

At such low temperature, however, the energy-loss rate of the non-relativistic neutron is negligible. Thus, Eq. (B.19) is enough for our analysis. 


\section{C.1 Elastic scattering}

We consider the elastic scattering of an energetic nucleon $N_{i}(p$ or $n)$ with a background nucleus $A_{j}$ (background proton or $\alpha$ ) through the process $N_{i}\left(p_{i}\right)+A_{j}\left(p_{j}\right) \rightarrow N_{i}\left(p_{i}^{\prime}\right)+A_{j}\left(p_{j}^{\prime}\right)$, where we denoted the four-momentum in the parenthesises. Then, $p_{j}=\left(m_{H_{j}}, 0,0,0\right)$ and $p_{j}^{\prime}=\left(E_{j}^{\prime}, \boldsymbol{p}^{\prime}{ }_{j}\right)$, and the kinetic energy of the scattered target $(p$ or $\alpha)$ is given by

$$
K_{j}^{\prime}=E_{j}^{\prime}-m_{A_{j}}
$$

Using the Mandelstam variable $t$, which is given by

$$
t=\left(p_{j}^{\prime}-p_{j}\right)^{2}=-2 m_{A_{j}} K_{j}^{\prime},
$$

we obtain

$$
K_{j}^{\prime}=\frac{-t}{2 m_{A_{j}}} .
$$

Kinematically, the maximal possible value of $K_{j}^{\prime}$ is given by

$$
K_{j}^{\prime(\max )}=\frac{2 m_{A_{j}} K_{i}}{\left(m_{N_{i}}+m_{H_{j}}\right)^{2}+2 m_{A_{j}} K_{i}} .
$$

For the elastic scattering processes, the distribution of $t$ is well approximated by the following form with the slope parameter $B_{\mathrm{sl}}$

$$
\frac{1}{\sigma^{(\mathrm{el})}} \frac{d \sigma^{(\mathrm{el})}}{d t}=\left[\frac{1}{\sigma^{(\mathrm{el})}} \frac{d \sigma^{(\mathrm{el})}}{d t}\right]_{t=0} \exp \left(-B_{\mathrm{sl}}|t|\right)
$$

where $\sigma^{(\mathrm{el})}$ is the cross section.

For the process $p p \rightarrow p p$, an accurate fitting formula of $B_{\mathrm{sl}}$ as a function of the centerof-mass energy $\sqrt{s}$ is given in Ref. [87. The error of the fit is within $10 \%$. In Fig. 46] we plot $B_{\mathrm{sl}}$ for $p p \rightarrow p p$ as a function of $\sqrt{s}$. We use the fitting formula given in Ref. [87] to calculate the slope parameter. ${ }^{\# 19}$ We also adopt the same formula for the elastic processes in which the projectile is neutron (i.e., $n p_{\mathrm{BG}} \rightarrow n p$ ). Notice that the Coulomb correction factor for the cross section is within $6 \%$ for $K_{n}=100 \mathrm{MeV}\left(15 \%\right.$ for $\left.K_{n}=10 \mathrm{MeV}\right)$. Therefore, the error of the transfered energy $K_{p}^{\prime}$ for $n p \rightarrow n p$ should be within $20 \%$. With Eq. (C.5), we can estimate the averaged transfered energy as

$$
\left\langle K_{j}^{\prime}\right\rangle \equiv \int d t K_{j}^{\prime} \frac{1}{\sigma^{(\mathrm{el})}} \frac{d \sigma^{(\mathrm{el})}}{d t} \sim \frac{1}{2 m_{A_{j}} B_{\mathrm{sl}}}
$$

\#19 We use the fitting formula even for $2 \mathrm{GeV} \lesssim \sqrt{s}<4 \mathrm{GeV}$ although primarily the formula was derived for $\sqrt{s} \gtrsim 4 \mathrm{GeV}$ [87. Note that the experimental data of the cross sections suggest that the energy dependence of $B_{\mathrm{sl}}$ is very mild. 


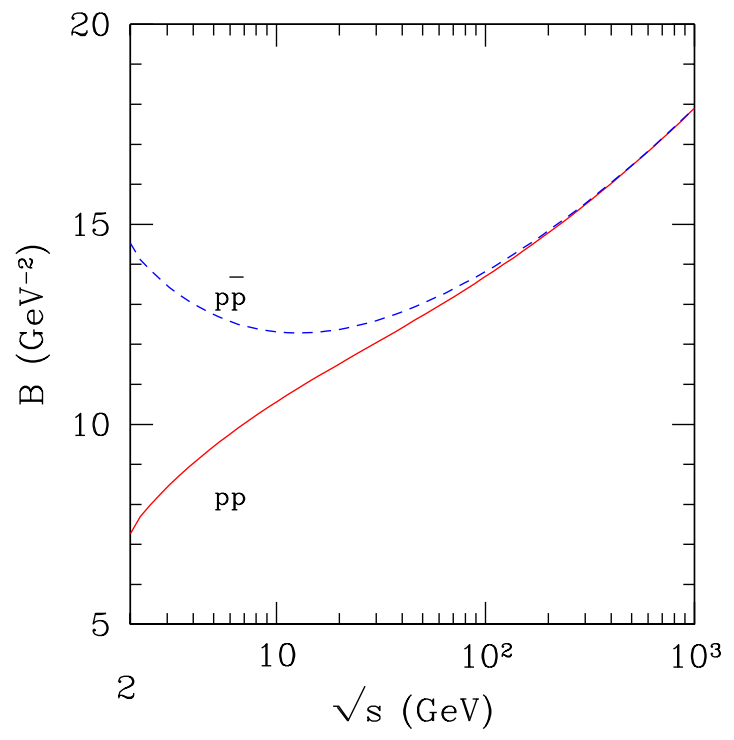

Figure 46: $B_{\mathrm{sl}}$ as a function of the center-of-mass energy $\sqrt{s}$. The solid (dashed) line denotes the slope parameter for the $p p \rightarrow p p(p \bar{p} \rightarrow p \bar{p})$ process. Here, the fitting formula given in [87] is used.

For example, for the process $p p_{\mathrm{BG}} \rightarrow p p$ with $K_{p}=5 \mathrm{GeV}$, we can use this formula to obtain $\left\langle K_{p}^{\prime}\right\rangle \sim 60 \mathrm{MeV}$.

For the elastic $p \alpha$ scattering, we use $B_{\mathrm{sl}}=28 \mathrm{GeV}^{-2}$ [88, which is derived from the experiment with the energetic proton with its kinetic energy $695-991 \mathrm{MeV}$. Although we neglect the energy-dependence of $B_{\mathrm{sl}}$ for the elastic scattering processes with $\alpha_{\mathrm{BG}}$, such approximation would not significantly affect our results. This is because the averaged value of the transfered energy through $p \alpha \rightarrow p \alpha$ process is $\left\langle K_{\alpha}^{\prime}\right\rangle \sim 5 \mathrm{MeV}$ and the energy transfer in this process is less efficient than $p p_{\mathrm{BG}} \rightarrow p p$. We also adopt this value in case of the elastic scattering of high energy neutron, $n \alpha \rightarrow n \alpha$.

Then, from Eq. (C.5), we derive the energy distribution of the final-state $A_{j}$ as

$$
f_{N_{i}+A_{j} \rightarrow N_{i}+A_{j}}^{A_{j}}\left(K_{i}, K_{j}^{\prime}\right)=\frac{2 m_{A_{j}} B_{\mathrm{sl}}}{1-\exp \left(-B_{\mathrm{sl}}|t|^{(\max )}\right)} \exp \left(-2 m_{A_{j}} B_{\mathrm{sl}} K_{j}^{\prime}\right),
$$

where $|t|^{(\max )}=2 m_{A_{j}} K_{j}^{\prime(\max )}$ is the maximal possible value of $|t|$. In our study, we use the above formula to evaluate the energy distribution of the scattered $A_{i}$. Notice that the energy distribution of the $N_{i}$ in the final state can be derived by using the relation $K_{i}^{\prime}=K_{i}-K_{j}^{\prime}$ :

$$
f_{N_{i}+A_{j} \rightarrow N_{i}+A_{j}}^{N_{i}}\left(K_{i}, K_{i}^{\prime}\right)=\frac{2 m_{A_{j}} B_{\mathrm{sl}}}{1-\exp \left(-B_{\mathrm{sl}}|t|^{(\max )}\right)} \exp \left[-2 m_{A_{j}} B_{\mathrm{sl}}\left(K_{i}-K_{i}^{\prime}\right)\right] .
$$




\section{C.2 Inelastic scattering}

In this subsection, we discuss the inelastic scattering process of an energetic nucleon $N_{i}$ with a background nucleus $A_{j}$. As we have discussed in the previous subsection, the differences of cross sections for the $p p$ and $n p$ processes are small. Therefore we apply the formulae obtained for the $p p$ and $p \alpha$ collisions to $n p$ and $n \alpha$ collisions, respectively.

Since we sometimes use the Lorentz transformation from the center-of-mass frame to the rest frame of the target particle, it is convenient to define the Lorentz factor as a function of the kinetic energy of the beam nucleon. Explicit expression of the Lorentz factor is given by

$$
\gamma^{(\mathrm{CM})}\left(K_{i}\right)=\frac{\left(m_{N_{i}}+m_{A_{j}}\right)+K_{i}}{E^{(\mathrm{CM})}\left(K_{i}\right)},
$$

where the energy in the center-of-mass frame is given by

$$
E^{(\mathrm{CM})}\left(K_{i}\right)=\sqrt{\left(m_{N_{i}}+m_{A_{j}}\right)^{2}+2 m_{A_{j}} K_{i}} .
$$

\section{C.2.1 Inelastic scattering with background proton}

First, we consider the inelastic scattering between high-energy proton (neutron) with background proton. The most important inelastic processes are the ones with one pion production: $p+p_{\mathrm{BG}} \rightarrow p+p(n)+\pi$ ( or $\left.n+p_{\mathrm{BG}} \rightarrow n+p(n)+\pi\right)$. Indeed, the cross sections of these processes are much larger than those of other inelastic processes. Hereafter, we consider the process $p\left(p_{i}\right)+p_{\mathrm{BG}}\left(p_{j}\right) \rightarrow p\left(p_{i}^{\prime}\right)+N_{j}\left(p_{j}^{\prime}\right)+\pi\left(p_{\pi}^{\prime}\right)$.

In order to study the energy distribution of $p$ in the final state (i.e., distribution of $K_{i}^{\prime}$ ), it is useful for us to introduce Feynman's $x$-parameter. Let us decompose the threemomentum $\boldsymbol{p}_{i}^{\prime}$ as

$$
\boldsymbol{p}_{i}^{\prime}=\boldsymbol{p}_{i, \|}^{\prime}+\boldsymbol{p}_{i, \perp}^{\prime},
$$

where $\boldsymbol{p}_{i, \|}^{\prime}$ and $\boldsymbol{p}_{i, \perp}^{\prime}$ are components of $\boldsymbol{p}_{i}^{\prime}$ parallel to and perpendicular to the direction of the initial-state energetic proton, respectively. Then, the Feynman's $x$-parameter is defined as

$$
x \equiv \frac{p_{i, \|}^{\prime}}{p_{i, \|}^{\prime(\max )}},
$$

where $p_{i, \|}^{\prime}=\left|\boldsymbol{p}_{i, \|}^{\prime}\right|$ and $p_{i, \|}^{\prime(\max )}$ is the maximal possible value of $p_{i, \|}^{\prime}$. From experimental data on the high-energy inclusive $p p$ reactions, it is known that the distribution of $x$ is approximately independent of $\sqrt{s}$ (called "Feynman scaling" [89, 90]). Indeed, various experiments reported that the kinetic energy of the final-state proton $K_{i}^{\prime}$ in the inclusive pion production process has flat distribution (see, for e.g., [91]). Thus, with such experimental 
result, we estimate the averaged value of $x$ as

$$
\langle x\rangle=\frac{\int_{0}^{1} x \frac{d \sigma^{(\mathrm{incl})}}{d x} d x}{\int_{0}^{1} \frac{d \sigma^{(\mathrm{incl})}}{d x} d x} \sim 0.5 .
$$

Using the above result, we parameterize the energy of the beam particle (i.e., $p$ ) in the final state using the "inelasticity" $\kappa_{p}$ :

$$
K_{i}^{\prime}=\left(1-\kappa_{p}\right) K_{i}
$$

Notice that, for high-energy pion production processes, $x \simeq 1-\kappa_{p}$. ${ }^{\# 20}$ In our analysis, we approximate that $\kappa_{p}$ is constant and use $\kappa_{p}=0.5$ for the numerical calculations. \#21 That is, we approximate that the fraction of the energy-loss of the initial-state energetic proton is always $\kappa_{p}$. We also use the above arguments in case of the energetic neutron injection. Then, we obtain the energy distribution of the final-state $p$ as

$$
f_{\left(p, p_{\mathrm{BG}} ; 2\right)}^{p}\left(K_{i}, K_{i}^{\prime}\right)=f_{\left(p, p_{\mathrm{BG}} ; 3\right)}^{p}\left(K_{i}, K_{i}^{\prime}\right)=\delta\left(K_{i}^{\prime}-\left(1-\kappa_{p}\right) K_{i}\right) .
$$

In order to study the energy distribution of other final-state particles, we can use the experimental data of the momentum distribution of the daughter particles from the $p p$ collision. In particular, if we consider some daughter particle $H_{k}$ produced by the $p p$ collision, energy distribution is well fitted by the following formula with the inverse slope parameter $K_{T}$ [92, 93]:

$$
\frac{1}{M_{\mathrm{T}}} \frac{d \sigma^{(\text {inel })}}{d M_{\mathrm{T}} d y d \phi_{\mathrm{a}}} \propto \exp \left(-M_{\mathrm{T}} / K_{T}\right),
$$

where $M_{T}$ is the transverse mass which is give by

$$
M_{\mathrm{T}}^{2}=m_{H_{k}}^{2}+\left|\boldsymbol{p}_{H_{k}, \perp}^{\prime}\right|^{2}
$$

$y$ is the rapidity and $\phi_{a}$ is the azimuthal angle. Remarkably, for any kind of the daughter particles such as protons, pions, and the other mesons, Eq. (C.16) provides a good approximation. In addition, the inverse slope parameter is not sensitive to $\sqrt{s}$.

It is notable that $K_{T}$ provides an typical kinetic energy of the daughter particles in the CM frame since $\left\langle M_{\mathrm{T}}\right\rangle \sim K_{T}$. This is interpreted as the consequence of the fact that the fragmentation scheme into hadrons is controlled by QCD and that $K_{T}$ represents the

\footnotetext{
\#20 Although, strictly speaking, the relation $x \simeq 1-\kappa_{p}$ holds in the CM frame, it can be approximately satisfied even in laboratory system for high-energy collision.

${ }^{\# 21}$ We have checked that, for the $20 \%$ variation of the $\kappa$-parameters, $\xi$-parameters change at most 15 $\%$. Such uncertainty is relatively small compared to the total uncertainty of the $\xi$-parameters due to the errors of the hadronic cross sections (see section 91). Thus, in our analysis, errors from the $\kappa$-parameters are neglected.
} 
typical energy scale of the fragmentation. Using the results of the resent experiments of high-energy $p p$ and $p^{7}$ Be collisions, we adopt the following value for $K_{T}$ [94, 95]:

$$
K_{T}=140 \pm 15 \mathrm{MeV} \text {. }
$$

From the distribution of $M_{\mathrm{T}}$, we estimate the distribution of the kinetic energy of the scattered target $N_{j}$. Unfortunately, direct information of $\left|\boldsymbol{p}_{j, \|}^{\prime}\right|$ is not available. Thus we assume that, at the high-energy scattering processes we are interested in, the daughter particles are distributed isotropically in CM system. With this assumption, we estimate the averaged value of the kinetic energy of $N_{j}$ as

$$
\left\langle K_{j}^{\prime}\right\rangle_{p p}=\gamma^{(\mathrm{CM})}\left(K_{i}\right) K_{T}+\left[\gamma^{(\mathrm{CM})}\left(K_{i}\right)-1\right] m_{N} .
$$

For simplicity, we approximate that the scattered target always have the kinetic energy $\left\langle K_{j}^{\prime}\right\rangle_{p p}$ and hence the distribution of the scattered particle is written as

$$
f_{\left(p, p_{\mathrm{BG}} ; 2\right)}^{p}\left(K_{i}, K_{j}^{\prime}\right)=f_{\left(p, p_{\mathrm{BG}} ; 3\right)}^{n}\left(K_{i}, K_{j}^{\prime}\right)=\delta\left(K_{j}^{\prime}-\left\langle K_{j}^{\prime}\right\rangle_{p p}\right) .
$$

In a similar fashion, we can also estimate the averaged kinetic energy of the produced pion as

$$
\left\langle K_{\pi}^{\prime}\right\rangle_{p p}=\gamma^{(\mathrm{CM})}\left(K_{i}\right) K_{T}+\left[\gamma^{(\mathrm{CM})}\left(K_{i}\right)-1\right] m_{\pi} .
$$

However, for pions, the time scale for the hadronic scattering processes is always longer than that of the electromagnetic stopping processes or the lifetime. Thus, we do not have to consider the energetic pions.

\section{C.2.2 Inelastic scattering with $\alpha_{\mathrm{BG}}$}

Next we consider the energy transfer in the inelastic processes with background $\alpha_{\mathrm{BG}}$. In this process, the target particle $\alpha_{\mathrm{BG}}$ may or may not destroyed so we have to consider various final-states. As in the case of the inelastic scattering with the background proton, we denote the process as $p\left(p_{i}\right)+\alpha_{\mathrm{BG}}\left(p_{j}\right) \rightarrow p\left(p_{i}^{\prime}\right)+N_{j}\left(p_{j}^{\prime}\right)+\pi\left(p_{\pi}^{\prime}\right)+\cdots$.

For the energy distribution of the injected particle in the final state, we use the similar treatment as the inelastic $p p$ collision. That is, we define

$$
K_{i}^{\prime}=\left(1-\kappa_{\alpha}\right) K_{i}
$$

where $\kappa_{\alpha}$ is the inelasticity related to ${ }^{4} \mathrm{He}$. As in the $p p$ collision case, we approximate that $\kappa_{\alpha}$ is a constant and use the distribution function

$$
f_{\left(p, \alpha_{\mathrm{BG}} ; 2\right)}^{p}\left(K_{i}, K_{i}^{\prime}\right)=f_{\left(p, \alpha_{\mathrm{BG}} ; 3\right)}^{p}\left(K_{i}, K_{i}^{\prime}\right)=\delta\left(K_{i}^{\prime}-\left(1-\kappa_{\alpha}\right) K_{i}\right) .
$$

Since we do not have sufficient data to estimate $\kappa_{\alpha}$, we assume that $\kappa_{\alpha}=\kappa_{p}$ in our analysis. \#22

\footnotetext{
\#22In the so-called "Cascade Model," where a nucleus such as "He is approximately treated as a composition of many "independent" nucleons with uniform density, an approximate relation $\kappa_{\alpha} \sim$ $1-\left(1-\kappa_{p}\right)^{1.3-1.43}$ holds (see Ref. [96] and references therein). If we adopt $\kappa_{p} \sim 0.5$, we obtain $\kappa_{\alpha} \sim 0.59-0.63$ and the error in our treatment is about $20 \%$.
} 
Now we consider the daughter nuclei produced by the inelastic scattering processes with $\alpha_{\mathrm{BG}}$. In our study, energy distribution of the final-state nuclei is mostly approximated as follows. If kinematically allowed, we assume that all the produced debris nuclei $A_{k}$ (except the injected proton) have kinetic energy

$$
\left\langle K_{k}^{\prime}\right\rangle_{p \alpha}=\gamma^{(\mathrm{CM})}\left(K_{i}\right) K_{T}+\left[\gamma^{(\mathrm{CM})}\left(K_{i}\right)-1\right] m_{A_{k}},
$$

where we used the transverse-mass distribution given in Eq. (C.16). (The total energy is assumed to be conserved by the pion emissions.) If $K_{i}$ is not large enough, the total kinetic energy of the final-state particles becomes larger than $K_{i}$ with the above approximation. In such cases, we assume equipartition of the total momentum in the CM system. Therefore, our approximation is surely conservative than the case that we always assume the equipartition at every moment.

In the study of the shower evolution and the surviving probability of the light elements produced by the hadrodissociation of $\alpha_{\mathrm{BG}}$, we use the energy distribution obtained by the above procedure. For some processes, however, experimental data provides better information about the energy distributions. In particular for the processes $n+\alpha_{\mathrm{BG}} \rightarrow$ $\mathrm{T}+\cdots$ and $n+\alpha_{\mathrm{BG}} \rightarrow{ }^{3} \mathrm{He}+\cdots$, the daughter $\mathrm{T}$ and ${ }^{3} \mathrm{He}$ play the role of the "spectator" particle and their typical energies are known to be very small $(\sim \mathcal{O}(1) \mathrm{MeV})$ [69. For these processes, the energies of $\mathrm{T}$ and ${ }^{3} \mathrm{He}$ are overestimated if we naively use our shower algorithm. \#23 For the process $n+\alpha \rightarrow \mathrm{T}+\cdots$, the experimental data is available and we found that the energy distribution is the final-state $\mathrm{T}$ is well approximated by

$$
\frac{1}{\sigma_{n+\alpha \rightarrow \mathrm{T}+\cdots}} \frac{d \sigma_{n+\alpha \rightarrow \mathrm{T}+\cdots}}{d E_{\mathrm{T}}} \simeq 0.09831 \mathrm{MeV}^{-1} \times \exp \left(-\frac{E_{\mathrm{T}}}{5.789 \mathrm{MeV}}\right),
$$

independently of the energy of the beam particle. The energetic non-thermally produced $\mathrm{T}$ and ${ }^{3} \mathrm{He}$ play very significant roles in the study of the non-thermally produced ${ }^{6} \mathrm{Li}$. In calculating the number of the non-thermally produced ${ }^{6} \mathrm{Li}$, we adopt Eq. (C.25) as energy distributions of $\mathrm{T}$ and ${ }^{3} \mathrm{He}$ produced by the hadrodissociation processes.

\section{Method for the Numerical Calculation}

In this appendix, we explain how we study the evolution of the hadronic shower in our numerical calculation. In our approximation, once a high-energy hadron $H_{i}$ is injected into the thermal bath with the initial energy $E_{H_{i}}^{(\text {in) }}$, its energy is first decreased down to $\tilde{E}_{H_{i}}^{(R=1)}$, then scatters off the background nuclei (in our case, proton or $\alpha_{\mathrm{BG}}$ ). Then, the energy distribution of the final-state particles after the hadronic scattering process is given by

\footnotetext{
\#23 Since the energy of $\mathrm{T}$ and ${ }^{3} \mathrm{He}$ may be overestimated in the study of the shower evolution, the energy of the final-state $p$ and $n$ may be underestimated in some case. This may be the case when the $K_{i}$ is so small that the equipartition of the total momentum in the CM system is adopted. Then, such an error is expected to be small since, in such a case, energy of the nuclei are inversely proportional to their masses. In addition, our treatment is also justified from the point of view of obtaining conservative constraints.
} 
the distribution function $G_{H_{i} \rightarrow H_{j}}$ defined in Eq. (17.3) (or $\tilde{G}_{N_{i} \rightarrow N_{j}}$ given in Eq. (7.9) with our approximation). The evolution of the hadronic shower can be in principle solved by using Eq. (7.6) once the initial spectra of the primordial hadrons directly emitted from $X$ is given. Since our purpose is to count the number of the non-thermally produced/dissociated light elements, however, there is rather a simple method which we use in our numerical calculation. In our numerical calculation, we first prepare the energy bins for kinetic energy of each relevant hadrons (in our method, we prepare the energy bins for proton, neutron and $\left.{ }^{4} \mathrm{He}\right)$. Hereafter, we consider the case where the $I$-th energy bin has the center value $E_{I}$ and the width $\Delta E_{I}$. (Thus, $E_{I} \pm \frac{1}{2}\left(\Delta E_{I}+\Delta E_{I \pm 1}\right)=E_{I \pm 1}$.) Here, $I$ runs from 0 to $N_{\text {bin }}$ where 0 -th bin is for stopped hadrons while $N_{\text {bin }}$-th bin is for hadrons with maximal possible energy. Then, we calculate the accumulated number of hadrons fallen into each energy bin during the evolution of the hadronic shower.

As we mentioned in Section [7] in our approximation, evolution of the hadronic shower is studied by following the energy-loss processes of the proton and the neutron. Thus, the first thing to do is to calculate the number of the nucleon $N_{j}$ scattered into $J$-th bin when one nucleon $N_{i}$ is injected into $I$-th bin. By using $\tilde{G}$ given in Eq. (7.9), we can calculate such transfer matrix, which we call $\tilde{T}_{H_{i}, H_{j}}^{I, J}$, as

$$
\tilde{T}_{N_{i}, N_{j}}^{I, J}=\tilde{G}_{N_{i} \rightarrow N_{j}}\left(E_{I}, E_{J} ; T\right) \Delta E_{J},
$$

where $\tilde{E}_{N_{i}}^{(R=1)}$ satisfies the relation $R^{N_{i}}\left(E_{I}, \tilde{E}_{N_{i}}^{(R=1)} ; T\right)=1$. Since, in our case, the energy of the nucleon always decreases after the hadronic scattering processes, $\tilde{T}_{N_{i}, N_{j}}^{I, J}$ vanishes if $I \leq J$. \#24

Next, we consider the case where one $N_{i}$ is injected into $I$-th bin, and estimate the accumulated number of $N_{j}$ fallen into $J$-th bin after (infinite number of) multiple hadronic scattering processes. We call such quantity as $\tilde{U}_{N_{i}, N_{j}}^{I, J}$. Using the fact that $\tilde{T}_{N_{i}, N_{j}}^{I, J}=0$ for $I \leq J$, the following relation holds

$$
\tilde{U}_{N_{i}, N_{j}}^{I, J}=\delta_{I J} \delta_{i j}+\sum_{K=J+1}^{I-1} \sum_{N_{k}} \tilde{T}_{N_{i}, N_{k}}^{I, K} \tilde{U}_{N_{k}, N_{j}}^{K, J} .
$$

Thus, using the above relation, we can recursively determine $\tilde{U}_{N_{i}, N_{j}}^{I, J}$ from $I=J=0$ by increasing $I$ and $J$.

For the hadronic decay of $X$, then the accumulated number of $N_{j}$ fallen into $J$-th bin is calculated as

$$
\tilde{S}_{N_{j}}\left(E_{J}\right) \Delta E_{J} \equiv \sum_{l=0}^{\infty} \tilde{F}_{N_{j}}^{(l)}\left(E_{J}\right) \Delta E_{J}=\sum_{I} \sum_{N_{i}} F_{N_{i}}^{(0)}\left(E_{I}\right) \Delta E_{I} \tilde{U}_{N_{i}, N_{j}}^{I, J},
$$

where $F_{N_{i}}^{(0)}$ is the distribution function of $N_{i}$ emitted from the hadronic decay of $X$. Substituting this relation into Eqs. (17.12) and (17.14), we calculate the $\xi$-parameters for the light elements.

${ }^{\# 24}$ Strictly speaking, $\tilde{T}_{N_{i}, N_{j}}^{I, I}$ does not vanish since the energy bin has some finite width. In our analysis, such correction is taken into account. 


\section{E Dissociation of Non-thermally Produced Li}

Here, we discuss the scattering and dissociation of the non-thermally produced Li (and $\mathrm{Be}$ ). Non-thermally produced ${ }^{6} \mathrm{Li}$ and ${ }^{7} \mathrm{Li}$ with energy of the order of $(1-10) \mathrm{MeV}$ can be destroyed by scattering off background particles before being stopped. Thus, in order to estimate the present abundance of ${ }^{6} \mathrm{Li}$ and ${ }^{7} \mathrm{Li}$, it is necessary to understand the surviving probability of these nuclei.

As other charged nuclei, the energetic Li loses its kinetic energy during the propagation in the thermal bath by scattering off the background $e$ and $\gamma$. Since the scattering rate (and the energy-loss rate) of ${ }^{6} \mathrm{Li}$ is much larger than the expansion rate of the universe, the evolution of the number density of the non-thermally produced ${ }^{6} \mathrm{Li}$ is governed by

$$
\frac{1}{n_{\mathrm{Li} \text { (N.T.) }}} \frac{d n_{\mathrm{Li}(\text { N.T.) }}}{d t}=-n_{p} \sigma_{\mathrm{Li}+p \rightarrow \ldots} \beta_{\mathrm{Li}}
$$

where $\sigma_{\mathrm{Li}+p \rightarrow \ldots}$ is the cross section for the Li dissociation process. Thus, the surviving rate of Li with its initial energy $E_{\mathrm{Li}}^{(\mathrm{in})}$ is calculated as $\# 25$

$$
\begin{aligned}
P_{\mathrm{Li} \rightarrow \mathrm{Li}}\left(E_{\mathrm{Li}}^{(\mathrm{in})}\right) & =\exp \left[-\int_{0}^{\infty} d t n_{p} \sigma_{\mathrm{Li}+p \rightarrow \ldots} \beta_{\mathrm{Li}}\right] \\
& =\exp \left[-\int_{E_{\mathrm{Li}+p \rightarrow \ldots}^{(\mathrm{th})}}^{E_{\mathrm{ii}}^{(\mathrm{in})}} d E_{\mathrm{Li}}\left(\frac{d E_{\mathrm{Li}}}{d t}\right)^{-1} n_{p} \sigma_{\mathrm{Li}+p \rightarrow \ldots} \beta_{\mathrm{Li}}\right],
\end{aligned}
$$

where $\left(d E_{\mathrm{Li}} / d t\right)$ is the energy-loss rate of $\mathrm{Li}$, and $E_{\mathrm{Li}+p \rightarrow \ldots}^{(\mathrm{th})}$ is the threshold energy for the destruction process of Li.

In the calculation of the surviving probability of ${ }^{6} \mathrm{Li}$, the dominant dissociation process is ${ }^{6} \mathrm{Li}\left(p,{ }^{3} \mathrm{He}\right)^{4} \mathrm{He}$. \#26 Cross section for this process is given in Refs. [83, 72]. For the readers' convenience, we show the plot of the cross section for this process in Fig. 47.

We numerically evaluate the surviving rate for ${ }^{6} \mathrm{Li}$ for various values of its initial energy. In Fig. 48, we plot the contours of constant surviving probability $P^{6}{ }_{\mathrm{Li} \rightarrow}{ }^{6} \mathrm{Li}$. As one can see, for $T \lesssim 50 \mathrm{keV}$ when the thermal process ${ }^{6} \mathrm{Li}\left(p,{ }^{4} \mathrm{He}\right){ }^{3} \mathrm{He}$ becomes inefficient, the

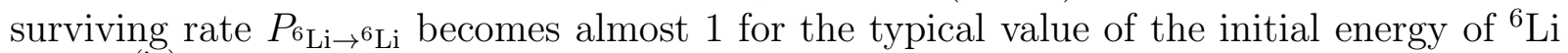
(i.e., $\left.E_{\mathrm{Li}}^{\text {(in })} \sim(5-10) \mathrm{MeV}\right)$.

For non-thermally produced ${ }^{7} \mathrm{Li}$, the cross sections for the processes ${ }^{7} \mathrm{Li}\left(p,{ }^{4} \mathrm{He}\right){ }^{4} \mathrm{He}$ and ${ }^{7} \operatorname{Li}\left(p,{ }^{3} \mathrm{He}\right) X$ are available. We use the cross sections for these processes given in Refs. [84, 83, 72] and Ref. [85, 72, respectively. Then, we calculate $P^{{ }^{7} \mathrm{Li} \rightarrow{ }^{7} \mathrm{Li}}$ and found that $P_{7} \mathrm{Li} \rightarrow{ }^{7} \mathrm{Li}$ is also close to 1 for the cases we are interested in.

Finally, we comment on the surviving rate of non-thermally produced ${ }^{7} \mathrm{Be}$. Unfortunately, the cross sections for the dissociation processes of non-thermally produced ${ }^{7} \mathrm{Be}$ is

\#25 In fact, dissociation process ${ }^{6} \mathrm{Li}\left(p,{ }^{3} \mathrm{He}\right){ }^{4} \mathrm{He}$ may occur even after ${ }^{6} \mathrm{Li}$ is thermalized. Such an effect is taken into account in the $\mathrm{BBN}$ code we use and hence the surviving rate $P^{6} \mathrm{Li} \rightarrow{ }^{6} \mathrm{Li}$ parameterizes the dissociation of ${ }^{6} \mathrm{Li}$ before thermalization.

\#26 There is another possible dissociation process of ${ }^{6} \mathrm{Li}(p, \gamma){ }^{7} \mathrm{Be}$. Cross section for this process is, however, much smaller than that for ${ }^{6} \mathrm{Li}\left(p,{ }^{3} \mathrm{He}\right){ }^{4} \mathrm{He}$ [72, and hence this process is irrelevant. 


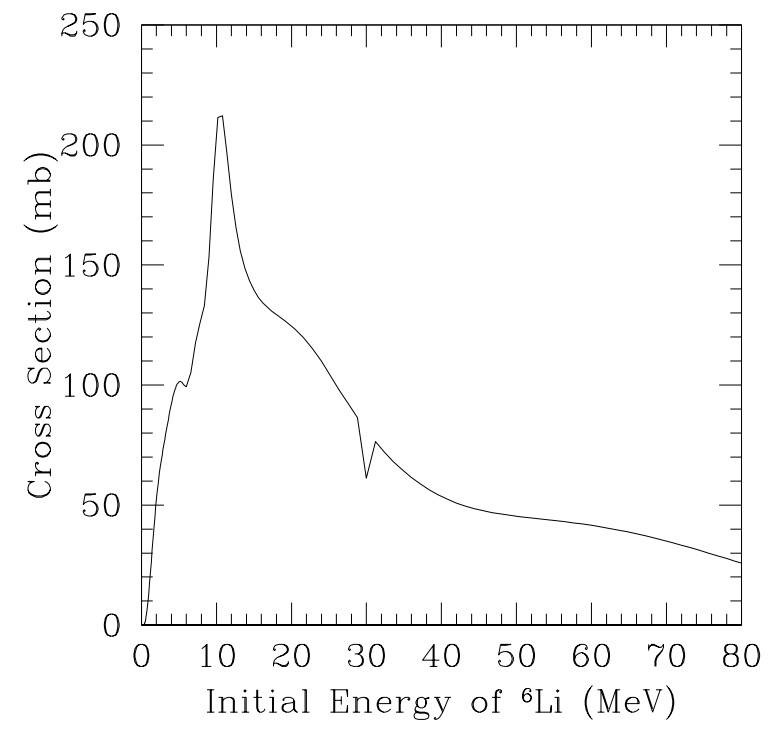

Figure 47: Cross section for the process ${ }^{6} \mathrm{Li}\left(p,{ }^{3} \mathrm{He}\right){ }^{4} \mathrm{He}$ as a function of the initial energy of ${ }^{6} \mathrm{Li}$. For the original data, see Refs. [83, 72].

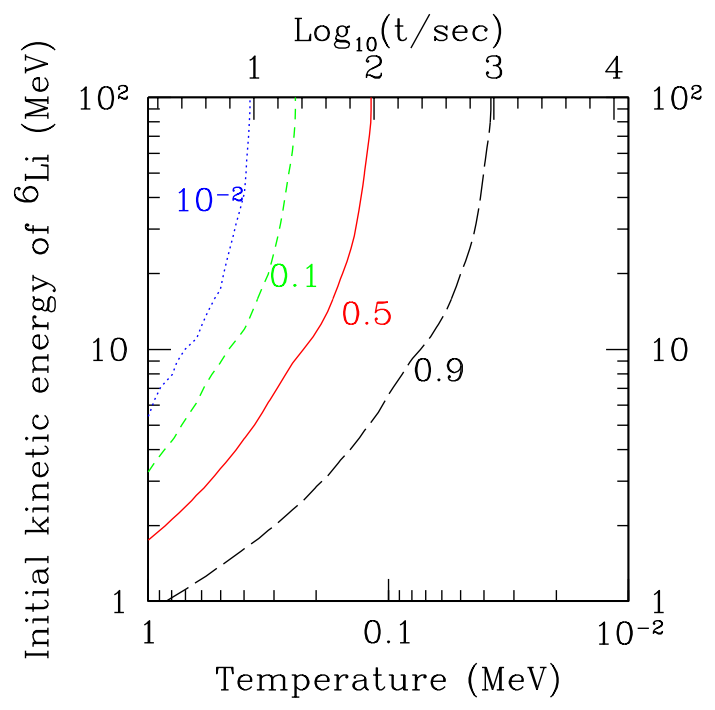

Figure 48: Contours of constant $P_{6 \mathrm{Li} \rightarrow}{ }^{6} \mathrm{Li}$. The horizontal axis is the background temperature while the vertical axis is the initial kinetic energy of ${ }^{6} \mathrm{Li}$. 
not available. Thus, we use the dissociation cross sections of ${ }^{7} \mathrm{Li}$ also for the dissociation processes of ${ }^{7} \mathrm{Be}$.

Although the surviving rate $P^{6}{ }_{\mathrm{Li}} \rightarrow{ }^{6} \mathrm{Li}$ is almost 1 for the case where the non-thermal production of ${ }^{6} \mathrm{Li}$ may become significant, we include $P^{6} \mathrm{Li} \rightarrow{ }^{6} \mathrm{Li}$ in the calculation of the number of non-thermally produced ${ }^{6} \mathrm{Li}$. Same is true for ${ }^{7} \mathrm{Li}$ and ${ }^{7} \mathrm{Be}$ when non-thermal production of these light elements are discussed.

\section{F Primordial Abundance of Gravitino}

In this appendix, we evaluate the primordial abundance of the gravitino in the inflationary universe. In particular, we calculate the gravitino abundance as a function of the welldefined reheating temperature $T_{\mathrm{R}}$. Compared to the old result given in Ref. [9], there are two major improvements: (i) we have included the effects of the gravitino production during the period when the inflaton field is still oscillating (i.e, in the so-called dilute plasma), and (ii) for the gravitino production cross section in the thermal bath, formula taking account of the thermal mass of the gauge bosons is used in order to avoid the infrared singularity.

Gravitino is the gauge field for the local supersymmetry, and hence it couples to the supercurrent as

$$
\begin{aligned}
\mathcal{L}_{\psi J}= & -\frac{1}{\sqrt{2} M_{*}} D_{\nu} \phi^{*} \bar{\psi}_{\mu} \gamma^{\nu} \gamma^{\mu} \chi_{R}-\frac{1}{\sqrt{2} M_{*}} D_{\nu} \phi \bar{\chi}_{L} \gamma^{\mu} \gamma^{\nu} \psi_{\mu} \\
& -\frac{i}{8 M_{*}} \bar{\psi}_{\mu}\left[\gamma^{\nu}, \gamma^{\rho}\right] \gamma^{\mu} \lambda F_{\nu \rho}
\end{aligned}
$$

where $\chi_{R}$ and $\phi$ are fermion and boson in chiral multiplets, $\lambda$ is the gaugino, and $F_{\nu \rho}$ is the field strength of the gauge field. (Here, $D_{\nu}$ denotes the covariant derivative and $\chi_{R}$ satisfies $\left(1-\gamma_{5}\right) \chi_{R}=0$.)

Importantly, the interaction of the gravitino is suppressed by the inverse of $M_{*}$ and hence the gravitino interacts very weakly compared to the standard-model particles. In particular, if the temperature is lower than $\sim M_{*}$, gravitino is not thermalized in the expanding universe. Although it is out of the thermal bath, however, gravitinos are produced via the scattering processes of the thermal particles. In the expanding universe, evolution of the number density of the gravitino is governed by the following Boltzmann equation

$$
\frac{d n_{3 / 2}}{d t}+3 H n_{3 / 2}=\left\langle\sigma_{\text {tot }} v_{\text {rel }}\right\rangle n_{\text {rad }}^{2}
$$

where $\left\langle\sigma_{\text {tot }} v_{\text {rel }}\right\rangle$ is the "thermally averaged" total cross section (times relative velocity), and $n_{\text {rad }}=\frac{\zeta(3)}{\pi^{2}} T^{3}$. With the Lagrangian (F.1), the gravitino production cross sections are typically $\mathcal{O}\left(g_{i}^{2} / 32 \pi M_{*}^{2}\right)$, where $g_{i}$ denotes the gauge coupling constant for $S U(3)_{C}$, $S U(2)_{L}$ and $U(1)_{Y}$ for $i=3,2$ and 1 , respectively.

Approximated formula for the primordial gravitino abundance is obtained by integrating Eq. ( $(\underline{F .2})$ from the highest temperature in the radiation-dominated epoch, which 
approximately corresponds to the "reheating temperature" $T_{\mathrm{R}}$ after the inflation. Using the fact that the gravitino production cross section has very weak dependence on $T$, Eq. (F.2) can be easily solved. In order to parameterize the primordial abundance of the gravitino, it is convenient to define the "yield variable" as \#27

$$
Y_{3 / 2} \equiv \frac{n_{3 / 2}}{s}
$$

where $s$ is the entropy density which is given by

$$
s=\frac{2 \pi^{2}}{45} g_{* S} T^{3},
$$

where $g_{* S}$ is the effective number of the massless degrees of freedom. For the particle content of the minimal supersymmetric standard model (MSSM), $g_{* S}=228.75$ for temperature much higher than the masses of the superparticles while $g_{* S}=43 / 11$ for $T \ll m_{e}$. As we see just below, gravitino production processes are effective when $T \sim T_{\mathrm{R}}$ and hence, when $T \ll T_{\mathrm{R}}, Y_{3 / 2}$ becomes constant (as far as the total entropy in the comoving volume is conserved). Then, integrating Eq. (E.2) from $T=T_{\mathrm{R}}$ to $T \ll T_{\mathrm{R}}$, we obtain

$$
Y_{3 / 2} \sim \frac{45 \zeta(3)}{2 \pi^{4}}\left[\frac{\left\langle\sigma_{\mathrm{tot}} v_{\mathrm{rel}}\right\rangle n_{\mathrm{rad}}}{g_{* S} H}\right]_{T=T_{\mathrm{R}}} .
$$

The important point is that the primordial abundance of the gravitino is (approximately) proportional to the reheating temperature after the inflation. Thus, gravitinos are overproduced in many cases if the reheating temperature is too high.

For the detailed calculation of $Y_{3 / 2}$, however, the definition of the "reheating temperature" used in the above calculation is quite ambiguous since, in realistic models of slow-roll inflation, there exists a period when the universe is dominated by the oscillating energy of the inflaton field before the radiation-dominated epoch. (We call this period as the inflaton-dominated period.) Due to the decay of the inflaton field, the inflatondominated epoch evolves into the radiation-dominated epoch. The transition from the inflaton-dominated epoch to the radiation-dominated epoch occurs when the expansion rate of the universe is comparable to the decay rate of the inflaton. In this paper, we define the "reheating temperature" as

$$
T_{\mathrm{R}} \equiv\left(\frac{10}{g_{*} \pi^{2}} M_{*}^{2} \Gamma_{\mathrm{inf}}^{2}\right)^{1 / 4}
$$

where $g_{*}=g_{* S}$. (In estimating $T_{\mathrm{R}}$, we use $g_{*}=228.75$.) Notice that $T_{\mathrm{R}}$ given above is the same as the reheating temperature derived with the approximation such that $X$ instantaneously decays when the relation $3 H=\Gamma_{\text {inf }}$ is realized.

\#27 The yield variable used in this paper differs from that in [9] which defined $Y_{3 / 2}^{(\mathrm{KM})} \equiv n_{3 / 2} / n_{\text {rad }}$. We use $Y_{3 / 2}$ defined in Eq. (F.3) since, with this definition, $Y_{3 / 2}$ is independent of time for the temperature $T \ll T_{\mathrm{R}}$ as far as there is no entropy production. Notice that, for $T \ll m_{e}, Y_{3 / 2}^{(\mathrm{KM})} \simeq 14 Y_{3 / 2}$. 
Then, in order to take account of the effects of the gravitino production in the inflatondominated epoch, we numerically solve the Boltzmann equations. The Boltzmann equation for the gravitino abundance is given in Eq. (F.2), while evolutions of the energy densities of the radiation $\rho_{\text {rad }}$ and inflaton $\rho_{\mathrm{inf}}$ are governed by the following Boltzmann equations

$$
\begin{aligned}
& \frac{d \rho_{\mathrm{rad}}}{d t}+4 H \rho_{\mathrm{rad}}=\Gamma_{\mathrm{inf}} \rho_{\mathrm{inf}}, \\
& \frac{d \rho_{\mathrm{inf}}}{d t}+3 H \rho_{\mathrm{inf}}=-\Gamma_{\mathrm{inf}} \rho_{\mathrm{inf}},
\end{aligned}
$$

where $\Gamma_{\text {inf }}$ is the decay rate of the inflaton, and $\rho_{\text {rad }}$ is related to the background temperature as

$$
\rho_{\mathrm{rad}}=\frac{\pi^{2}}{30} g_{*} T^{4} .
$$

The thermally averaged cross section for the gravitino production is calculated in Ref. [78]. \#28 For $S U(N)$ super Yang-Mills model with $n_{\mathrm{f}}$ pairs of fundamental and anti-fundamental chiral superfields, we obtain \#29

$$
\begin{aligned}
\left\langle\sigma_{\text {tot }} v_{\text {rel }}\right\rangle= & {\left[1+\left(\frac{m_{\tilde{g}}^{2}}{3 m_{3 / 2}^{2}}\right)\right] \frac{3 g^{2}\left(N^{2}-1\right)}{32 \pi M_{*}^{2}} } \\
& \times \frac{\pi^{2}}{\zeta(3)}\left\{\left[\ln \left(T^{2} / m_{g, \text { th }}^{2}\right)+0.3224\right]\left(N+n_{\mathrm{f}}\right)+0.5781 n_{\mathrm{f}}\right\},
\end{aligned}
$$

where $m_{\tilde{g}}$ is the gaugino mass and $m_{g, \text { th }}$ is the thermal mass of the gauge boson which is given as

$$
m_{g, \mathrm{th}}^{2}=\frac{1}{6} g^{2}\left(N+n_{\mathrm{f}}\right) T^{2} .
$$

As the gaugino mass becomes larger, more gravitinos are produced. In our calculation, we calculate the gravitino abundance in the limit of $m_{\tilde{g}} \rightarrow 0$ to derive a conservative constraint. Thus, in our calculation, the gravitino abundance is underestimated by $\mathcal{O}(10) \%$ when the gaugino masses are comparable to the gravitino mass. If the gauginos are much heavier than the gravitino, upper bound on $T_{\mathrm{R}}$ is approximately obtained by rescaling the results by the factor $\left(m_{\tilde{g}_{3}}^{2} / 3 m_{3 / 2}^{2}\right)^{-1}$ using the fact that the gravitino production is dominated by processes related to the $S U(3)_{C}$ gauge fields.

\footnotetext{
\#28In the gravitino production cross section, infrared singularities arise due to the $t$ - and $u$-channel exchanges of the gauge bosons. In the previous calculation of $\left\langle\sigma_{\text {tot }} v_{\text {rel }}\right\rangle$ given in [9], such singularities were smeared by introducing a cut-off parameter for the scattering angle while, in [78, it is treated by properly taking account of the thermal mass of the gauge bosons. Numerically, difference of the thermally averaged cross sections given in Refs. 9] and 78 is at $10 \%$ level for most of the parameter space.

${ }^{\# 29}$ For $U(1)$ gauge interactions, $\left(N^{2}-1\right) \rightarrow 1,\left(N+n_{\mathrm{f}}\right) \rightarrow n_{\mathrm{f}}$, and $n_{\mathrm{f}}$ becomes the sum of the squared of the hypercharges of chiral multiplets. We thank Arnd Brandenburg for helpful correspondence on this point.
} 
We follow the evolutions of $n_{3 / 2}, \rho_{\text {rad }}$, and $\rho_{\text {inf }}$ from the period with $H \gg \Gamma_{\text {inf }}$ to $H \ll \Gamma_{\text {inf }}$ by numerically solving the Boltzmann equations (F.2), (F.7) and (F.8). In calculating $\left\langle\sigma_{\text {tot }} v_{\text {rel }}\right\rangle$, we sum over the contributions from all the MSSM gauge groups, $S U(3)_{C}, S U(2)_{L}$ and $U(1)_{Y}$. Then, we calculate $Y_{3 / 2}$ at $H \ll \Gamma_{\text {inf }}$ (i.e., $T \ll T_{\mathrm{R}}$ ). As we mentioned, $Y_{3 / 2}$ is approximately proportional to $T_{\mathrm{R}}$; more precisely, we found that the resultant gravitino abundance is well approximated by the following formula

$$
\begin{aligned}
Y_{3 / 2} \simeq & 1.9 \times 10^{-12} \\
& \times\left(\frac{T_{\mathrm{R}}}{10^{10} \mathrm{GeV}}\right)\left[1+0.045 \ln \left(\frac{T_{\mathrm{R}}}{10^{10} \mathrm{GeV}}\right)\right]\left[1-0.028 \ln \left(\frac{T_{\mathrm{R}}}{10^{10} \mathrm{GeV}}\right)\right] .
\end{aligned}
$$

\section{References}

[1] S. Weinberg, Phys. Rev. Lett. 48, 1303 (1982).

[2] T. Moroi, arXiv:hep-ph/9503210.

[3] T. Moroi, H. Murayama and M. Yamaguchi, Phys. Lett. B 303, 289 (1993).

[4] J. L. Feng, A. Rajaraman and F. Takayama, Phys. Rev. Lett. 91, 011302 (2003); Phys. Rev. D 68 (2003) 063504; J. L. Feng, S. f. Su and F. Takayama, arXiv:hep-ph/0404198.

[5] D. Lindley, Astrophys. J. 294 (1985) 1; M. Y. Khlopov and A. D. Linde, Phys. Lett. B 138, 265 (1984); F.Balestra et al., Sov. J. Nucl. Phys. 39, 626 (1984); M. Yu. Khlopov, Yu. L. Levitan, E. V. Sedelnikov and I. M. Sobol, Phys. Atom. Nucl. 571393 (1994); J. R. Ellis, J. E. Kim and D. V. Nanopoulos, Phys. Lett. B 145, 181 (1984). R. Juszkiewicz, J. Silk and A. Stebbins, Phys. Lett. B 158, 463 (1985); J. R. Ellis, D. V. Nanopoulos and S. Sarkar, Nucl. Phys. B 259 (1985) 175; J. Audouze, D. Lindley and J. Silk, Astrophys. J. 293, L53 (1985); D. Lindley, Phys. Lett. B 171 (1986) 235; M. Kawasaki and K. Sato, Phys. Lett. B 189, 23 (1987); R. J. Scherrer and M. S. Turner, Astrophys. J. 331 (1988) 19; J. R. Ellis et al., Nucl. Phys. B 373, 399 (1992).

[6] R. Dominguez-Tenreiro, Astrophys. J. 313, 523 (1987).

[7] M. H. Reno and D. Seckel, Phys. Rev. D 37, 3441 (1988).

[8] S. Dimopoulos, R. Esmailzadeh, L. J. Hall and G. D. Starkman, Astrophys. J. 330, 545 (1988); Phys. Rev. Lett. 60, 7 (1988); Nucl. Phys. B 311, 699 (1989).

[9] M. Kawasaki and T. Moroi, Prog. Theor. Phys. 93, 879 (1995); Astrophys. J. 452, 506 (1995). 
[10] M. Kawasaki and T. Moroi, Phys. Lett. B 346, 27 (1995).

[11] R. J. Protheroe, T. Stanev and V. S. Berezinsky, Phys. Rev. D 51, 4134 (1995).

[12] E. Holtmann, M. Kawasaki, K. Kohri and T. Moroi, Phys. Rev. D 60, 023506 (1999).

[13] K. Jedamzik, Phys. Rev. Lett. 84, 3248 (2000).

[14] M. Kawasaki, K. Kohri and T. Moroi, Phys. Rev. D 63, 103502 (2001).

[15] K. Kohri, Phys. Rev. D 64 (2001) 043515.

[16] R. H. Cyburt, J. R. Ellis, B. D. Fields and K. A. Olive, Phys. Rev. D 67, 103521 (2003).

[17] M. Kawasaki, K. Kohri and T. Moroi, arXiv:astro-ph/0402490.

[18] M. Kawasaki, K. Kohri and N. Sugiyama, Phys. Rev. D 62, 023506 (2000).

[19] K. Kohri and J. Yokoyama, Phys. Rev. D 61, 023501 (2000).

[20] T. Sjostrand, Comput. Phys. Commun. 82, 74 (1994).

[21] D. N. Spergel et al., Astrophys. J. Suppl. 148, 175 (2003).

[22] D. Kirkman et al., Astrophys. J. Suppl. 149, 1 (2003).

[23] D. Tytler, X. m. Fan and S. Burles, Nature 381, 207 (1996); S. Burles and D. Tytler, Astrophys. J. 499, 699 (1998).

[24] S. Burles and D. Tytler, Astrophys. J. 507, 732 (1998).

[25] J. M. O’Meara et al., Astrophys. J. 552, 718 (2001).

[26] M. Pettini and D. V. Bowen, Astrophys. J. 560, 41 (2001).

[27] J. K. Webb et al., Nature 388, 250 (1997).

[28] B. D. Fields and K. A. Olive, Astrophys. J. 506 177, (1998).

[29] Y. I. Izotov and T. X. Thuan, Astrophys. J. 602, 200 (2004).

[30] K. A. Olive and G. Steigman, Astrophys. J. Suppl. 97, 49 (1995).

[31] K. A. Olive, E. Skillman and G. Steigman, Astrophys. J. 483, 788 (1997).

[32] Y. I. Izotov, T. X. Thuan and V. A. Lipovetsky, Astrophys. J. Suppl. 108, 1 (1997).

[33] K. A. Olive and E. D. Skillman, arXiv:astro-ph/0405588 
[34] P. Bonifacio et al., Astron. Astrophys. 390, 91 (2002).

[35] B. D. Fields, K. Kainulainen, K. A. Olive, and D. Thomas, New Astron. 1, 7 (1996).

[36] S. G. Ryan, J. Norris, and T. C. Beers, Astrophys. J. 523, 654 (1999).

[37] S.G. Ryan et al., Astrophys. J. Lett. 530, L57 (2000).

[38] D. K. Duncan, D. L. Lambert, and M. Lemke, Astrophys. J. 401, 584 (1992); M. Cassé, R. Lehoucq, and E. Vangioni-Flam, Nature 373, 318 (1995); R. Ramaty, B. Kozlovsky, and R. E .Lingenfelter, Astrophys. J. Lett. 438, L21 (1995).

[39] M. Lemoine, D. N. Schramm, J. W. Truran, and C. J. Copi, Astrophys. J. 478, 554 (1997).

[40] B. D. Fields and K. A. Olive, New Astron. 4, 255 (1999).

[41] R. Ramaty, S. T. Scully, R. E. Ligenfelter, and B. Kozlovsky, Astrophys. J. 534, 747 (2000).

[42] T. K. Suzuki and S. Inoue, Astrophys. J. 573, 168 (2002).

[43] V. V. Smith, D. L. Lambert, and P. E. Nissen, Astrophys. J. 408, 262 (1993); L. M. Hobbs and J. A. Thorburn, Astrophys. J. 491, 772 (1997); V. V. Smith, D. L. Lambert, and P. E. Nissen, Astrophys. J. 506, 923 (1998); R. Cayrel et al., Astron. Astrophys. 343923 (1999).

[44] W. Aoki et al., submitted to Astron. and Astrophys. (2004).

[45] J. Geiss, "Origin and Evolution of the Elements," edited by N. Prantzos, E. VangioniFlam, and M. Cassé (Cambridge University Press, 1993) 89.

[46] C. Angulo et al. [NACRE collaboration], Nucl. Phys. A656, 3 (1999)

[47] R. H. Cyburt, B. D. Fields and K. A. Olive, New Astron. 6, 215 (2001).

[48] R. H. Cyburt, Phys. Rev. D 70, 023505 (2004).

[49] M. S. Smith, L. H. Kawano, and R. A. Malaney, Astrophys. J. Suppl. 85, 219 (1993).

[50] L.H. Kawano, preprint FERMILAB-Pub-92/04-A (1992).

[51] C. Brune et al., Phys. Rev. C60, 015801 (1999).

[52] G. M. Hale et al., ENDF/B-VI Evaluation, Material 125, Revision 1 (1991).

[53] K. M. Nollett and S. Burles, Phys. Rev. D62, 123505 (2000).

[54] E. Vangioni-Flam, A. Coc, and M. Cassé, Astron. Astrophys. 360, 15 (2000). 
[55] K. Ichikawa, M. Kawasaki and F. Takahashi, Phys. Lett. B 597, 1 (2004).

[56] R.D. Evans, "The Atomic Nucleus," (McGraw-Hill, 1955).

[57] R. Pfiffer, Z. Phys. 208, 129 (1968).

[58] D. D. Faul, B. L. Berman, P. Mayer and D. L. Olson, Phys. Rev. Lett. 44, 129 (1980).

[59] A. N. Gorbunov and A. T. Varfolomeev, Phys. Lett. 11, 137 (1964).

[60] Yu. M. Arkatov et al., Sov. J. Nucl. Phys. 19, 589 (1974).

[61] J. D. Irish et al., Can. J. Phys. 53, 802 (1975).

[62] C. K. Malcolm, D. B. Webb, Y. M. Shin and D. M. Skopik, Phys. Lett. B 47, 433 (1973).

[63] V. P. Denisov, A. P. Komar, L. A. Kul'chitskii and E. D. Makhnovskii, Sov. J. Nucl. Phys. 5, 349 (1967).

[64] B.L. Berman, Atomic Data and Nuclear Data Tables 15, 319 (1975).

[65] V. P. Denisov and L. A. Kul'chitskii, Sov. J. Nucl. Phys. 5, 344 (1967).

[66] K. Hagiwara et al. [Particle Data Group Collaboration], Phys. Rev. D 66, 010001 (2002).

[67] J. Bijnens and F. Cornet, Nucl. Phys. B 296, 557 (1988).

[68] V.B. Berestetskii, E.M. Lifshitz and L.P. Pitaevskii, "Quantum Electrodynamics," (Pergamon press, 1982).

[69] J. P. Meyer, Astron. Astrophys. Suppl. 7417 (1972).

[70] See, for e.g., L. I. Schiff, "Quantum Mechanics," (McGraw-Hill, 1968).

[71] J. A. Koepke and R. E. Brown, Phys. Rev. C16, 18 (1977).

[72] Experimental Nuclear Reaction Data (EXFOR/CSISRS) homepage, http://www.nndc.bnl.gov/exfor/index.html.

[73] B. G. Glagola et al, Phys. Rev. Lett. 411698 (1978).

[74] S. M. Read and V. E. Viola, Jr., Atomic data and nuclear data tables 31, 359 (1984).

[75] B. E. J. Pagel, "Nucleosynthesis and Chemical Evolution of Galaxies," (Cambridge University Press, 1997). 
[76] K. Jedamzik, arXiv:astro-ph/0402344.

[77] L. M. Krauss, Nucl. Phys. B 227, 556 (1983).

[78] M. Bolz, A. Brandenburg and W. Buchmuller, Nucl. Phys. B 606, 518 (2001).

[79] L. Randall and R. Sundrum, Nucl. Phys. B 557 (1999) 79; G. F. Giudice, M. A. Luty, H. Murayama and R. Rattazzi, JHEP 9812 (1998) 027; J. A. Bagger, T. Moroi and E. Poppitz, JHEP 0004 (2000) 009.

[80] K. Kohri, M. Yamaguchi and J. Yokoyama, Phys. Rev. D70, 043522 (2004), arXiv:hep-ph/0403043.

[81] M. Fukugita and T. Yanagida, Phys. Lett. B 174 (1986) 45.

[82] S. Davidson and A. Ibarra, Phys. Lett. B 535, 25 (2002); G. F. Giudice et al., Nucl. Phys. B 685, 89 (2004); W. Buchmuller, P. Di Bari and M. Plumacher, arXiv:hep-ph/0406014.

[83] S. N. Abramovich et al., Vop. At. Nauki i Tekhn., Ser. Yadernye Konstanty, 417 (1984).

[84] N. Jarmie and J. D. Seagrave, Los Alamos Scientific Lab. Reports, No.2014 (1957).

[85] N. A. Vlasov and A. M. Ogloblin, Nucl. Reactions at Low and Middle Energies, Moscow, 24 (1957).

[86] R. J. Gould, Astrophys. J. 417, 12 (1993).

[87] M. M. Block and R. N. Cahn, Phys. Lett. B 163, 250 (1985).

[88] O. G. Grenbenjuk et al., Nucl. Phys. A500, 637 (1989).

[89] R. P. Feynman, Phys. Rev. Lett. 23, 1415 (1969).

[90] M.L. Perl, "High Energy Hadron Physics," (John Wiley \& Sons, 1974).

[91] M. A. Abolins, G. A. Smith and M. Z. Ming, Phys. Rev. Lett. 25, 126 (1970).

[92] B. Alper et al. [British-Scandinavian Collaboration], Nucl. Phys. B 87, 19 (1975).

[93] C. Y. Wong, "Introduction To High-Energy Heavy Ion Collisions," (World Scientific, 1994).

[94] M. Kaneta [NA44 Collaboration], Prog. Theor. Phys. Suppl. 129, 167 (1997).

[95] M. Kaneta, Ph. D thesis (Hiroshima University, 1999).

[96] Y. Nara et al., Phys. Rev. C 61, 024901 (2000). 
[97] J. D. Jackson, "Classical Electrodynamics," (John Wiley \& Sons, 1962).

[98] H. Bethe and W. Heitler, Proc. Roy. Soc. Lond. A 146, 83 (1934).

[99] G. R. Blumenthal, Phys. Rev. D 1, 1596 (1970).

[100] M. J. Chodorowski, A. A. Adziarski, and M. Sikora, Astrophys. J. 400, 181(1992).

[101] V. S. Berezinsky and S. I. Grigor'eva, Astron. Astrophys. 199, 1 (1988). 